\title{
Measurement and QCD Analysis of the Diffractive Deep-Inelastic Scattering Cross Section at HERA
}

\author{
H1 Collaboration
}

\begin{abstract}
A detailed analysis is presented of the diffractive deep-inelastic scattering process $e p \rightarrow$ $e X Y$, where $Y$ is a proton or a low mass proton excitation carrying a fraction $1-x_{\mathbb{P}}>0.95$ of the incident proton longitudinal momentum and the squared four-momentum transfer at the proton vertex satisfies $|t|<1 \mathrm{GeV}^{2}$. Using data taken by the $\mathrm{H} 1$ experiment, the cross section is measured for photon virtualities in the range $3.5 \leq Q^{2} \leq 1600 \mathrm{GeV}^{2}$, triple differentially in $x_{\mathbb{P}}, Q^{2}$ and $\beta=x / x_{\mathbb{P}}$, where $x$ is the Bjorken scaling variable. At low $x_{I P}$, the data are consistent with a factorisable $x_{I P}$ dependence, which can be described by the exchange of an effective pomeron trajectory with intercept $\alpha_{\mathbb{P}}(0)=$ $1.118 \pm 0.008$ (exp.) ${ }_{-0.010}^{+0.029}$ (model). Diffractive parton distribution functions and their uncertainties are determined from a next-to-leading order DGLAP QCD analysis of the $Q^{2}$ and $\beta$ dependences of the cross section. The resulting gluon distribution carries an integrated fraction of around $70 \%$ of the exchanged momentum in the $Q^{2}$ range studied. Total and differential cross sections are also measured for the diffractive charged current process $e^{+} p \rightarrow \bar{\nu}_{e} X Y$ and are found to be well described by predictions based on the diffractive parton distributions. The ratio of the diffractive to the inclusive neutral current $e p$ cross sections is studied. Over most of the kinematic range, this ratio shows no significant dependence on $Q^{2}$ at fixed $x_{\mathbb{P}}$ and $x$ or on $x$ at fixed $Q^{2}$ and $\beta$.
\end{abstract}


A. Aktas 9 , V. Andreev ${ }^{25}$, T. Anthonis ${ }^{3}$, B. Antunovic ${ }^{26}$, S. Aplin ${ }^{9}$, A. Asmone ${ }^{33}$,

A. Astvatsatourov ${ }^{3}$, A. Babaev ${ }^{24, \dagger}$, S. Backovic ${ }^{30}$, A. Baghdasaryan $^{37}$, P. Baranov $^{25}$,

E. Barrelet ${ }^{29}$, W. Bartel ${ }^{9}$, S. Baudrand ${ }^{27}$, S. Baumgartner ${ }^{39}$, M. Beckingham ${ }^{9}$, O. Behnke ${ }^{12}$,

O. Behrendt ${ }^{6}$, A. Belousov ${ }^{25}$, N. Berger ${ }^{39}$, J.C. Bizot ${ }^{27}$, M.-O. Boenig ${ }^{6}$, V. Boudry ${ }^{28}$,

J. Bracinik $^{26}$, G. Brandt ${ }^{12}$, V. Brisson ${ }^{27}$, D. Bruncko ${ }^{15}$, F.W. Büsser ${ }^{10}$, A. Bunyatyan ${ }^{11,37}$,

G. Buschhorn ${ }^{26}$, L. Bystritskaya ${ }^{24}$, A.J. Campbell ${ }^{9}$, F. Cassol-Brunner ${ }^{21}$, K. Cerny ${ }^{32}$,

V. Cerny ${ }^{15,46}$, V. Chekelian ${ }^{26}$, J.G. Contreras ${ }^{22}$, J.A. Coughlan ${ }^{4}$, Y.R. Coppens ${ }^{2}$, B.E. Cox ${ }^{20}$,

G. Cozzika ${ }^{8}$, J. Cvach ${ }^{31}$, J.B. Dainton ${ }^{17}$, W.D. Dau ${ }^{14}$, K. Daum ${ }^{36,42}$, Y. de Boer ${ }^{24}$,

B. Delcourt ${ }^{27}$, M. Del Degan ${ }^{39}$, A. De Roeck ${ }^{9,44}$, E.A. De Wolf ${ }^{3}$, C. Diaconu ${ }^{21}$, V. Dodonov ${ }^{11}$,

A. Dubak $^{30,45}$, G. Eckerlin ${ }^{9}$, V. Efremenko ${ }^{24}$, S. Egli ${ }^{35}$, R. Eichler ${ }^{35}$, F. Eisele ${ }^{12}$, A. Eliseev ${ }^{25}$,

E. Elsen ${ }^{9}$, S. Essenov ${ }^{24}$, A. Falkewicz ${ }^{5}$, P.J.W. Faulkner ${ }^{2}$, L. Favart ${ }^{3}$, A. Fedotov ${ }^{24}$, R. Felst ${ }^{9}$,

J. Feltesse ${ }^{8}$, J. Ferencei ${ }^{15}$, L. Finke ${ }^{10}$, M. Fleischer ${ }^{9}$, G. Flucke ${ }^{33}$, A. Fomenko $^{25}$, G. Franke ${ }^{9}$,

T. Frisson ${ }^{28}$, E. Gabathuler ${ }^{17}$, E. Garutti ${ }^{9}$, J. Gayler ${ }^{9}$, C. Gerlich ${ }^{12}$, S. Ghazaryan ${ }^{37}$,

S. Ginzburgskaya ${ }^{24}$, A. Glazov ${ }^{9}$, I. Glushkov ${ }^{38}$, L. Goerlich ${ }^{5}$, M. Goettlich ${ }^{9}$, N. Gogitidze ${ }^{25}$,

S. Gorbounov ${ }^{38}$, C. Grab $^{39}$, T. Greenshaw ${ }^{17}$, M. Gregori ${ }^{18}$, B.R. Grell ${ }^{9}$, G. Grindhammer ${ }^{26}$,

C. Gwilliam ${ }^{20}$, D. Haidt ${ }^{9}$, M. Hansson ${ }^{19}$, G. Heinzelmann ${ }^{10}$, R.C.W. Henderson ${ }^{16}$,

H. Henschel ${ }^{38}$, G. Herrera ${ }^{23}$, M. Hildebrandt ${ }^{35}$, K.H. Hiller ${ }^{38}$, D. Hoffmann ${ }^{21}$,

R. Horisberger ${ }^{35}$, A. Hovhannisyan ${ }^{37}$, T. Hreus ${ }^{3,43}$, S. Hussain ${ }^{18}$, M. Ibbotson ${ }^{20}$, M. Ismail ${ }^{20}$, M. Jacquet ${ }^{27}$, X. Janssen ${ }^{3}$, V. Jemanov ${ }^{10}$, L. Jönsson ${ }^{19}$, C.L. Johnson ${ }^{2}$, D.P. Johnson ${ }^{3}$,

A.W. Jung ${ }^{13}$, H. Jung ${ }^{19,9}$, M. Kapichine ${ }^{7}$, J. Katzy ${ }^{9}$, I.R. Kenyon ${ }^{2}$, C. Kiesling ${ }^{26}$, M. Klein ${ }^{38}$,

C. Kleinwort ${ }^{9}$, T. Klimkovich ${ }^{9}$, T. Kluge ${ }^{9}$, G. Knies ${ }^{9}$, A. Knutsson ${ }^{19}$, V. Korbel ${ }^{9}$, P. Kostka ${ }^{38}$,

K. Krastev ${ }^{9}$, J. Kretzschmar ${ }^{38}$, A. Kropivnitskaya ${ }^{24}$, K. Krüger ${ }^{13}$, M.P.J. Landon ${ }^{18}$,

W. Lange ${ }^{38}$, G. Laštovička-Medin ${ }^{30}$, P. Laycock ${ }^{17}$, A. Lebedev ${ }^{25}$, G. Leibenguth ${ }^{39}$,

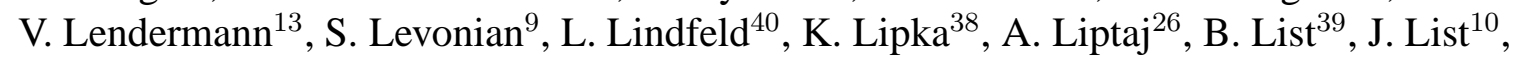

E. Lobodzinska ${ }^{38,5}$, N. Loktionova ${ }^{25}$, R. Lopez-Fernandez ${ }^{23}$, V. Lubimov ${ }^{24}$,

A.-I. Lucaci-Timoce ${ }^{9}$, H. Lueders ${ }^{10}$, T. Lux ${ }^{10}$, L. Lytkin ${ }^{11}$, A. Makankine ${ }^{7}$, N. Malden ${ }^{20}$,

E. Malinovski ${ }^{25}$, P. Marage ${ }^{3}$, R. Marshall ${ }^{20}$, L. Marti ${ }^{9}$, M. Martisikova ${ }^{9}$, H.-U. Martyn ${ }^{1}$,

S.J. Maxfield ${ }^{17}$, A. Mehta ${ }^{17}$, K. Meier ${ }^{13}$, A.B. Meyer ${ }^{9}$, H. Meyer ${ }^{36}$, J. Meyer ${ }^{9}$, V. Michels ${ }^{9}$,

S. Mikocki ${ }^{5}$, I. Milcewicz-Mika ${ }^{5}$, D. Milstead ${ }^{17}$, D. Mladenov ${ }^{34}$, A. Mohamed ${ }^{17}$, F. Moreau ${ }^{28}$,

A. Morozov ${ }^{7}$, J.V. Morris ${ }^{4}$, M.U. Mozer ${ }^{12}$, K. Müller ${ }^{40}$, P. Murín ${ }^{15,43}$, K. Nankov ${ }^{34}$,

B. Naroska ${ }^{10}$, Th. Naumann ${ }^{38}$, P.R. Newman ${ }^{2}$, C. Niebuhr ${ }^{9}$, A. Nikiforov ${ }^{26}$, G. Nowak ${ }^{5}$,

K. Nowak ${ }^{40}$, M. Nozicka ${ }^{32}$, R. Oganezov ${ }^{37}$, B. Olivier ${ }^{26}$, J.E. Olsson ${ }^{9}$, S. Osman ${ }^{19}$,

D. Ozerov ${ }^{24}$, V. Palichik ${ }^{7}$, I. Panagoulias 9 , T. Papadopoulou ${ }^{9}$, C. Pascaud $^{27}$, G.D. Patel ${ }^{17}$,

H. Peng ${ }^{9}$, E. Perez ${ }^{8}$, D. Perez-Astudillo ${ }^{22}$, A. Perieanu ${ }^{9}$, A. Petrukhin ${ }^{24}$, D. Pitzl ${ }^{9}$,

R. Plačakyte ${ }^{26}$, B. Portheault ${ }^{27}$, B. Povh ${ }^{11}$, P. Prideaux ${ }^{17}$, A.J. Rahmat ${ }^{17}$, N. Raicevic ${ }^{30}$,

P. Reimer ${ }^{31}$, A. Rimmer ${ }^{17}$, C. Risler ${ }^{9}$, E. Rizvi ${ }^{18}$, P. Robmann ${ }^{40}$, B. Roland ${ }^{3}$, R. Roosen ${ }^{3}$,

A. Rostovtsev ${ }^{24}$, Z. Rurikova ${ }^{26}$, S. Rusakov ${ }^{25}$, F. Salvaire ${ }^{10}$, D.P.C. Sankey ${ }^{4}$, M. Sauter ${ }^{39}$,

E. Sauvan ${ }^{21}$, F.-P. Schilling ${ }^{9,44}$, S. Schmidt ${ }^{9}$, S. Schmitt ${ }^{9}$, C. Schmitz ${ }^{40}$, L. Schoeffel $^{8}$,

A. Schöning ${ }^{39}$, H.-C. Schultz-Coulon ${ }^{13}$, F. Sefkow ${ }^{9}$, R.N. Shaw-West ${ }^{2}$, I. Sheviakov ${ }^{25}$,

L.N. Shtarkov ${ }^{25}$, T. Sloan ${ }^{16}$, P. Smirnov ${ }^{25}$, Y. Soloviev ${ }^{25}$, D. South ${ }^{9}$, V. Spaskov ${ }^{7}$, A. Specka ${ }^{28}$, M. Steder ${ }^{9}$, B. Stella ${ }^{33}$, J. Stiewe ${ }^{13}$, A. Stoilov ${ }^{34}$, U. Straumann ${ }^{40}$, D. Sunar ${ }^{3}$, V. Tchoulakov ${ }^{7}$,

G. Thompson ${ }^{18}$, P.D. Thompson ${ }^{2}$, T. Toll ${ }^{9}$, F. Tomasz ${ }^{15}$, D. Traynor ${ }^{18}$, T.N. Trinh ${ }^{21}$, P. Truöl ${ }^{40}$,

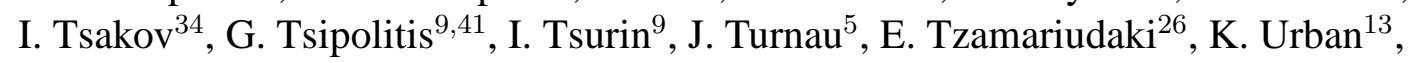

M. Urban ${ }^{40}$, A. Usik ${ }^{25}$, D. Utkin ${ }^{24}$, A. Valkárová ${ }^{32}$, C. Vallée ${ }^{21}$, P. Van Mechelen ${ }^{3}$, A. Vargas

Trevino $^{6}$, Y. Vazdik ${ }^{25}$, C. Veelken ${ }^{17}$, S. Vinokurova ${ }^{9}$, V. Volchinski ${ }^{37}$, K. Wacker ${ }^{6}$, G. Weber ${ }^{10}$, R. Weber ${ }^{39}$, D. Wegener ${ }^{6}$, C. Werner ${ }^{12}$, M. Wessels ${ }^{9}$, B. Wessling ${ }^{9}$, Ch. Wissing ${ }^{6}$, R. Wolf $^{12}$, 
E. Wünsch ${ }^{9}$, S. Xella ${ }^{40}$, W. Yan ${ }^{9}$, V. Yeganov ${ }^{37}$, J. Žáček ${ }^{32}$, J. Zálešák ${ }^{31}$, Z. Zhang ${ }^{27}$, A. Zhelezov ${ }^{24}$, A. Zhokin ${ }^{24}$, Y.C. Zhu ${ }^{9}$, J. Zimmermann ${ }^{26}$, T. Zimmermann ${ }^{39}$, H. Zohrabyan ${ }^{37}$, and F. Zomer ${ }^{27}$

${ }^{1}$ I. Physikalisches Institut der RWTH, Aachen, Germany ${ }^{a}$

${ }^{2}$ School of Physics and Astronomy, University of Birmingham, Birmingham, UK ${ }^{b}$

${ }^{3}$ Inter-University Institute for High Energies ULB-VUB, Brussels; Universiteit Antwerpen,

Antwerpen; Belgium ${ }^{c}$

${ }^{4}$ Rutherford Appleton Laboratory, Chilton, Didcot, $U K^{b}$

${ }^{5}$ Institute for Nuclear Physics, Cracow, Poland ${ }^{2}$

${ }^{6}$ Institut für Physik, Universität Dortmund, Dortmund, Germany ${ }^{a}$

${ }^{7}$ Joint Institute for Nuclear Research, Dubna, Russia

${ }^{8}$ CEA, DSM/DAPNIA, CE-Saclay, Gif-sur-Yvette, France

${ }^{9}$ DESY, Hamburg, Germany

${ }^{10}$ Institut für Experimentalphysik, Universität Hamburg, Hamburg, Germany ${ }^{a}$

${ }^{11}$ Max-Planck-Institut für Kernphysik, Heidelberg, Germany

${ }^{12}$ Physikalisches Institut, Universität Heidelberg, Heidelberg, Germany ${ }^{a}$

${ }^{13}$ Kirchhoff-Institut für Physik, Universität Heidelberg, Heidelberg, Germany ${ }^{a}$

${ }^{14}$ Institut für Experimentelle und Angewandte Physik, Universität Kiel, Kiel, Germany

${ }^{15}$ Institute of Experimental Physics, Slovak Academy of Sciences, Košice, Slovak Republic ${ }^{f}$

${ }^{16}$ Department of Physics, University of Lancaster, Lancaster, $U K^{b}$

${ }^{17}$ Department of Physics, University of Liverpool, Liverpool, $U K^{b}$

${ }^{18}$ Queen Mary and Westfield College, London, UK ${ }^{b}$

${ }^{19}$ Physics Department, University of Lund, Lund, Sweden ${ }^{g}$

${ }^{20}$ Physics Department, University of Manchester, Manchester, $U K^{b}$

${ }^{21}$ CPPM, CNRS/IN2P3 - Univ. Mediterranee, Marseille - France

${ }^{22}$ Departamento de Fisica Aplicada, CINVESTAV, Mérida, Yucatán, México ${ }^{j}$

${ }^{23}$ Departamento de Fisica, CINVESTAV, México ${ }^{j}$

${ }^{24}$ Institute for Theoretical and Experimental Physics, Moscow, Russia ${ }^{k}$

${ }^{25}$ Lebedev Physical Institute, Moscow, Russia ${ }^{e}$

${ }^{26}$ Max-Planck-Institut für Physik, München, Germany

${ }^{27}$ LAL, Université de Paris-Sud 11, IN2P3-CNRS, Orsay, France

${ }^{28}$ LLR, Ecole Polytechnique, IN2P3-CNRS, Palaiseau, France

${ }^{29}$ LPNHE, Universités Paris VI and VII, IN2P3-CNRS, Paris, France

${ }^{30}$ Faculty of Science, University of Montenegro, Podgorica, Serbia and Montenegro ${ }^{e}$

${ }^{31}$ Institute of Physics, Academy of Sciences of the Czech Republic, Praha, Czech Republic ${ }^{h}$

${ }^{32}$ Faculty of Mathematics and Physics, Charles University, Praha, Czech Republic ${ }^{h}$

${ }^{33}$ Dipartimento di Fisica Università di Roma Tre and INFN Roma 3, Roma, Italy

${ }^{34}$ Institute for Nuclear Research and Nuclear Energy, Sofia, Bulgaria ${ }^{e}$

${ }^{35}$ Paul Scherrer Institut, Villigen, Switzerland

${ }^{36}$ Fachbereich C, Universität Wuppertal, Wuppertal, Germany

${ }^{37}$ Yerevan Physics Institute, Yerevan, Armenia

${ }^{38}$ DESY, Zeuthen, Germany

${ }^{39}$ Institut für Teilchenphysik, ETH, Zürich, Switzerland ${ }^{i}$

${ }^{40}$ Physik-Institut der Universität Zürich, Zürich, Switzerland ${ }^{i}$ 
${ }^{41}$ Also at Physics Department, National Technical University, Zografou Campus, GR-15773 Athens, Greece

${ }^{42}$ Also at Rechenzentrum, Universität Wuppertal, Wuppertal, Germany

${ }^{43}$ Also at University of P.J. Šafárik, Košice, Slovak Republic

${ }^{44}$ Also at CERN, Geneva, Switzerland

${ }^{45}$ Also at Max-Planck-Institut für Physik, München, Germany

${ }^{46}$ Also at Comenius University, Bratislava, Slovak Republic

$\dagger$ Deceased

${ }^{a}$ Supported by the Bundesministerium für Bildung und Forschung, FRG, under contract numbers 05 H1 IGUA /1, 05 HI 1PAA /1, 05 HI 1PAB /9, 05 H1 1PEA /6, 05 H1 1VHA /7 and $05 \mathrm{HI} 1 \mathrm{VHB} / 5$

${ }^{b}$ Supported by the UK Particle Physics and Astronomy Research Council, and formerly by the UK Science and Engineering Research Council

${ }^{c}$ Supported by FNRS-FWO-Vlaanderen, IISN-IIKW and IWT and by Interuniversity Attraction Poles Programme, Belgian Science Policy

${ }^{d}$ Partially Supported by the Polish State Committee for Scientific Research, SPUB/DESY/P003/DZ 118/2003/2005

e Supported by the Deutsche Forschungsgemeinschaft

${ }^{f}$ Supported by VEGA SR grant no. 2/4067/ 24

${ }^{g}$ Supported by the Swedish Natural Science Research Council

${ }^{h}$ Supported by the Ministry of Education of the Czech Republic under the projects LC527 and INGO-1P05LA259

${ }^{i}$ Supported by the Swiss National Science Foundation

${ }^{j}$ Supported by CONACYT, México, grant 400073-F

${ }^{k}$ Partially Supported by Russian Foundation for Basic Research, grants 03-02-17291 and 04-02-16445 


\section{Introduction}

Quantum Chromodynamics (QCD) is well established as the gauge field theory of the strong interaction. However, it is only able to provide reliable predictions for scattering processes if they involve short distance partonic interactions, where perturbative methods may be applied. In contrast, hadronic scattering cross sections are dominated by soft interactions, to which perturbation theory is not applicable. In a large fraction of these soft interactions, often termed 'diffractive', one or both of the interacting hadrons remains intact. Such processes are commonly discussed in terms of exchanges with net vacuum quantum numbers, though the exact nature of these exchanges is not well known.

The observation of high transverse momentum jet production in diffractive $p \bar{p}$ scattering [1] introduced the possibility of understanding the diffractive exchange in terms of partons. The presence of processes of the type $e p \rightarrow e X p$ (figure 1) in deep-inelastic scattering (DIS) at low Bjorken- $x$ at the HERA collider [2] offers a uniquely well controlled environment in which to study the QCD properties and structure of diffraction. Several measurements of the semiinclusive cross section for this 'diffractive DIS' process have been made by the H1 [3-6] and ZEUS [7-11] collaborations.

\section{(a)}

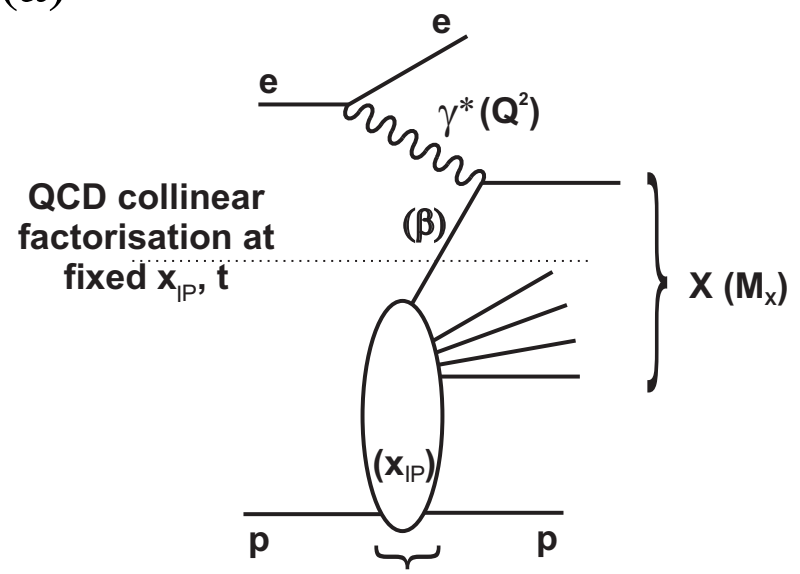

(b)

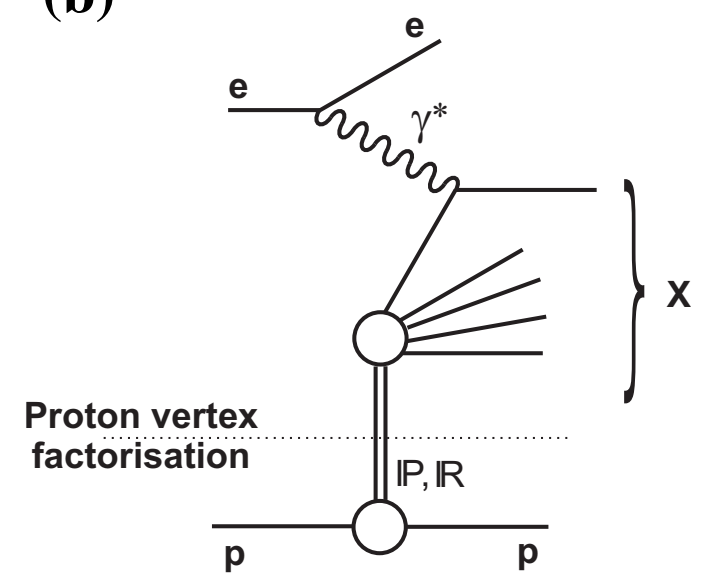

Figure 1: Schematic illustration of the neutral current diffractive DIS process $e p \rightarrow e X p$, proceeding via virtual photon exchange. The dotted lines in (a) and (b) show the points at which the diagram can be divided under the assumptions of QCD hard scattering collinear factorisation and proton vertex factorisation, respectively. The kinematic variables defined in section 2 are also indicated in (a).

The detailed explanation of hard diffraction has become a major challenge in the development of our understanding of the strong interaction at high energies and low $x$ values [12]. A wide variety of models has been put forward to interpret the dynamics of diffractive DIS as well as its relationships to inclusive DIS and to diffractive hadron-hadron scattering [13-19]. A general theoretical framework is provided by the proof [20] of a hard scattering QCD collinear factorisation theorem [21-23] for semi-inclusive DIS cross sections such as that for $e p \rightarrow e X p$. 
As illustrated in figure 1a, this theorem implies that the concept of 'diffractive parton distribution functions' (DPDFs) [22,24] may be introduced, representing conditional proton parton probability distributions under the constraint of a leading final state proton with a particular four-momentum. Empirically, a further factorisation has been found to apply to good approximation, whereby the variables which describe the proton vertex factorise from those describing the hard interaction $[5,6]$, as illustrated in figure $1 \mathrm{~b}$. According to this 'proton vertex' factorisation, the shape of the DPDFs is independent of the four-momentum of the final state proton. The dependence of the DPDF normalisation on the proton four-vector can be parameterised conveniently using Regge asymptotics, which amounts to a description of diffraction in terms of the exchange of a factorisable 'pomeron' $(\mathbb{P})$ [25] with universal parton densities [26].

Several authors have analysed diffractive DIS data to extract DPDFs [5, 10, 16, 27-32]. In $[5,6], \mathrm{H} 1$ measurements are studied, with the conclusion that the data are compatible with proton vertex factorisation at low fractional proton energy losses, $x_{\mathbb{P}}$. At larger $x_{\mathbb{P}}$, a separately factorisable sub-leading exchange $(\mathbb{R})$, with a different $x_{\mathbb{P}}$ dependence and partonic composition, is present. The DPDFs extracted in [5] are dominated by the gluon distribution. Further tests of the factorisation properties of diffractive DIS have been made by comparing predictions using these DPDFs with hadronic final state observables such as diffractive jet [33] and heavy quark [34] cross sections. These tests have shown a remarkable internal consistency within the HERA DIS data. In contrast, the DPDFs extracted in DIS are not expected to be directly applicable to hadron-hadron scattering [20-22,35]. Indeed diffractive factorisation breaks down spectacularly when the DPDFs from [5] are applied to diffractive $p \bar{p}$ interactions at the Tevatron [36]. However, with the introduction of an additional 'rapidity gap survival probability' factor to account for secondary interactions between the beam remnants [37], the HERA DPDFs remain an essential ingredient in the phenomenology of diffraction at the Tevatron and the LHC [38].

In this paper, a new measurement ${ }^{1}$ of the diffractive neutral current DIS cross section is presented. This is based upon $\mathrm{H} 1$ data for which there is an absence of hadronic activity in a large rapidity region extending close to the outgoing proton beam direction. It is thus complementary to measurements such as [6], in which the leading proton is detected and measured. The proton vertex factorisation property is tested and the dependence of the diffractive cross section on $x_{I P}$ is expressed in terms of an effective pomeron intercept $\alpha_{\mathbb{P}}(0)$. The dependence on $x$ and $Q^{2}$ is interpreted through a QCD analysis using the DGLAP [39] evolution equations at next-toleading order (NLO) [40], from which new DPDFs are determined. The kinematic range of validity of the DPDFs is tested systematically and, for the first time, an assessment is made of the experimental and theoretical uncertainties. A first measurement is also made of the diffractive charged current cross section, which is compared with a prediction based on the DPDFs extracted from the neutral current data. The ratio of diffractive to inclusive cross sections and its kinematic dependences are also investigated. Section 2 introduces the formalism adopted for the paper. Section 3 describes the cross section measurement. The results are presented in sections 4-7 and are followed by a summary in section 8 .

\footnotetext{
${ }^{1}$ The results presented here are in agreement with the previous $\mathrm{H} 1$ measurement using similar techniques [5] throughout most of the measured kinematic range. The new measurement and DPDFs supersede the old due to the improvements in precision, kinematic coverage, theoretical modelling and detector understanding.
} 


\section{Diffractive DIS Kinematic Variables and Observables}

The data studied here are subsets of inclusive H1 neutral and charged current DIS samples, arising from the processes $e^{+} p \rightarrow e^{+} X^{\prime}$ and $e^{+} p \rightarrow \bar{\nu}_{e} X^{\prime}$, where the positron (with fourmomentum $k$ ) couples to an electroweak gauge boson $(q)$, which interacts with the proton $(P)$. The usual DIS kinematic variables are defined as

$$
Q^{2}=-q^{2}, \quad x=\frac{-q^{2}}{2 P \cdot q}, \quad y=\frac{P \cdot q}{P \cdot k},
$$

where $Q^{2}$ is the boson virtuality, $x$ is the longitudinal momentum fraction of the proton carried by the struck quark and $y$ measures the inelasticity of the process. The squared invariant masses of the positron-proton and gauge boson-proton systems are $s=(k+P)^{2}$ and $W^{2}=(q+P)^{2}$, respectively.

The hadronic final state of any DIS event may be broken down into two systems $X$ and $Y$, separated by the largest gap in the rapidity distribution of the hadrons relative to an axis defined by the exchanged boson and the proton in their centre of mass frame [5]. If the masses $M_{\mathrm{X}}$ and $M_{\mathrm{Y}}$ of these two systems are small compared with the mass $W$ of the full hadronic final state, the two systems are expected to be separated by a large rapidity gap and a colourless exchange of well defined four-momentum may be considered to have taken place between them. As illustrated in figure 1a, the longitudinal momentum fractions, $x_{I P}$ of the colourless exchange with respect to the incoming proton, and $\beta$ of the struck quark with respect to the colourless exchange, are then defined by

$$
x_{\mathbb{P}}=\frac{q \cdot\left(P-p_{Y}\right)}{q \cdot P}, \quad \beta=\frac{Q^{2}}{2 q \cdot\left(P-p_{Y}\right)} .
$$

Here, $p_{Y}$ is the four-momentum of the $Y$ system and $\beta x_{I P}=x$. The squared four-momentum transferred at the proton vertex is

$$
t=\left(P-p_{Y}\right)^{2}
$$

The rapidity gap selection (section 3.4) implies that the data analysed in this paper are dominated by the case where $Y$ is a lone proton and $|t|$ is relatively small. However, since the system $Y$ is not detected directly, a small admixture of proton excitations and other systems such as leading neutrons is also present (see section 3.5).

The neutral current data are presented in the form of a 'diffractive reduced cross section' $\sigma_{r}^{D(3)}$, integrated over the ranges of $t$ and $M_{Y}$ specified in section 3.5 and related to the differential cross section measured experimentally by

$$
\frac{\mathrm{d}^{3} \sigma^{e p \rightarrow e X Y}}{\mathrm{~d} x_{\mathbb{P}} \mathrm{d} x \mathrm{~d} Q^{2}}=\frac{2 \pi \alpha^{2}}{x Q^{4}} \cdot Y_{+} \cdot \sigma_{r}^{D(3)}\left(x_{\mathbb{P}}, x, Q^{2}\right),
$$

where $Y_{+}=1+(1-y)^{2}$. Similarly to inclusive DIS [41], the reduced $e^{+} p$ cross section depends on the diffractive structure functions $F_{2}^{D(3)}$ and $F_{L}^{D(3)}$ in the one-photon exchange approximation according to

$$
\sigma_{r}^{D(3)}=F_{2}^{D(3)}-\frac{y^{2}}{Y_{+}} F_{L}^{D(3)} .
$$


For $y$ not too close to unity, $\sigma_{r}^{D(3)}=F_{2}^{D(3)}$ holds to very good approximation. In previous measurements of inclusive diffractive DIS at HERA, the data were presented in terms of $F_{2}^{D(3)}$ instead of $\sigma_{r}^{D(3)}$.

Due to the smaller available data sample, the charged current measurements must be integrated over some or all of the kinematic variables. They are presented as a total cross section and single differentially in either $x_{\mathbb{P}}, \beta$ or $Q^{2}$.

\section{Experimental Procedure}

\subsection{H1 Apparatus}

The $\mathrm{H} 1$ coordinate system is defined such that the origin is at the nominal $e p$ interaction point and the polar angle $\theta=0$ corresponds to the direction of the outgoing proton beam. The region $\theta<90^{\circ}$, which has positive pseudorapidity $\eta=-\ln \tan \theta / 2$, is referred to as the 'forward' hemisphere.

The interaction region is surrounded by the central tracking system, which consists of a silicon vertex detector, drift chambers and multi-wire proportional chambers, all located within a solenoidal magnetic field of $1.15 \mathrm{~T}$. The trajectories of charged particles are measured in the range $-1.7<\eta<1.7$ with a transverse momentum resolution of $\sigma\left(p_{T}\right) / p_{T} \simeq 0.005 p_{T} / \mathrm{GeV} \oplus$ 0.015. The Forward Tracking Detector and the Backward Drift Chamber (BDC) provide track segments of charged particles at smaller and larger $\theta$ than the central tracker, respectively.

A highly segmented Liquid Argon (LAr) sampling calorimeter, covering the range $-1.5<$ $\eta<3.4$, surrounds the tracking chambers and consists of electromagnetic and hadronic sections. The energy resolution is $\sigma(E) / E \simeq 11 \% / \sqrt{E / \mathrm{GeV}}$ for electrons and $\sigma(E) / E \simeq$ $50 \% / \sqrt{E / G e V}$ for hadrons, as obtained from test beam measurements [42]. The backward direction $(-4.0<\eta<-1.4)$ is covered by a lead / scintillating fibre calorimeter (SpaCal), which also has both electromagnetic and hadronic sections. The energy resolution for electrons is $\sigma(E) / E \simeq 7 \% / \sqrt{E / G e V}$.

In addition to the 'central detectors' described above, a set of 'forward detectors' are also used in the present analysis. The copper / silicon Plug calorimeter, the Forward Muon Detector (FMD) and the Proton Remnant Tagger (PRT) are sensitive to hadronic activity at large pseudorapidity, near to the outgoing proton beam. The Plug enables energy measurements to be made in the pseudorapidity range $3.5<\eta<5.5$. The FMD is a series of drift chambers covering the range $1.9<\eta<3.7$. Primary particles produced at larger $\eta$ are often detected indirectly in the FMD if they undergo a secondary scattering with the beam-pipe or other material. The PRT, a set of scintillators surrounding the beam pipe at $z=26 \mathrm{~m}$, detects charged particles in the region $6.0 \lesssim \eta \lesssim 7.5$.

The $e p$ luminosity is determined by measuring the rate of the QED Bremsstrahlung process $e p \rightarrow e p \gamma$ using a photon calorimeter close to the backward beam pipe at $z=-103 \mathrm{~m}$. A much more detailed description of the H1 apparatus can be found in $[43,44]$. 


\subsection{Data Samples}

Different event samples are used for different $Q^{2}$ ranges of the measurement, as summarised in table 1. For the interval $3<Q^{2}<13.5 \mathrm{GeV}^{2}$, a 'minimum bias' sample ('1997 MB') corresponding to an integrated luminosity of $2.0 \mathrm{pb}^{-1}$ is used, which was recorded during a dedicated data taking period in 1997 with unbiased triggers. For intermediate photon virtualities $\left(13.5<Q^{2}<105 \mathrm{GeV}^{2}\right)$, data taken throughout 1997 are used ('1997 all'), corresponding to an integrated luminosity of $10.6 \mathrm{pb}^{-1}$. The kinematic range $Q^{2}>133 \mathrm{GeV}^{2}$ is covered by a sample ('1999-2000') corresponding to $61.6 \mathrm{pb}^{-1}$, taken in the years 1999 and 2000.

For all three samples, HERA collided positrons with protons, the positron beam energy being $E_{e}=27.5 \mathrm{GeV}$ in each case. The proton beam energy was $E_{p}=820 \mathrm{GeV}$ in 1997 and $E_{p}=920 \mathrm{GeV}$ in 1999 and 2000, leading to ep centre of mass energies of $\sqrt{s}=301 \mathrm{GeV}$ and $\sqrt{s}=319 \mathrm{GeV}$, respectively. The ' $1997 \mathrm{MB}$ ' and ' 1997 all' samples are used to study neutral current interactions with the scattered electron ${ }^{2}$ detected in the SpaCal calorimeter. More details of the analysis of diffractive data with SpaCal electrons may be found in [45]. The '1999-2000' sample is used for the study of both neutral and charged current interactions. In the neutral current case, the scattered electron is detected in the LAr calorimeter. These measurements are further described in [46].

\begin{tabular}{|r|l|c|c|}
\hline \multicolumn{1}{|c|}{$Q^{2}$ range } & \multicolumn{1}{|c|}{ Data Set } & Proton Energy $E_{p}$ & Luminosity \\
\hline \hline $3<Q^{2}<13.5 \mathrm{GeV}^{2}$ & $1997 \mathrm{MB}$ & $820 \mathrm{GeV}$ & $2.0 \mathrm{pb}^{-1}$ \\
$13.5<Q^{2}<105 \mathrm{GeV}^{2}$ & 1997 all & $820 \mathrm{GeV}$ & $10.6 \mathrm{pb}^{-1}$ \\
$Q^{2}>133 \mathrm{GeV}^{2}$ & $1999-2000$ & $920 \mathrm{GeV}$ & $61.6 \mathrm{pb}^{-1}$ \\
\hline
\end{tabular}

Table 1: Summary of the data samples used in the analysis.

\subsection{Selection and Reconstruction of DIS Events}

The trigger conditions, detector alignment and calibration, and inclusive DIS selection criteria are very similar to those used in the analogous fully inclusive $\mathrm{H} 1$ analyses at low [47] and high [41] $Q^{2}$. The selection criteria are summarised below.

Neutral current DIS events are triggered by the detection of an energetic electromagnetic calorimeter cluster attributed to the scattered electron. Inefficient regions of the calorimeters, for example due to cracks between modules or poorly performing trigger cells, are not included in the analysis. The trigger efficiency is then close to $100 \%$ for the ranges in electron energy considered here, namely $E_{e}^{\prime}>6.5 \mathrm{GeV}$ for electrons detected in the SpaCal and $E_{e}^{\prime} \gtrsim 11 \mathrm{GeV}$ for LAr electrons. To suppress photoproduction background, in which the scattered electron escapes undetected in the backward direction and a hadron fakes the electron signature, cuts are applied on the lateral extent and isolation of the cluster forming the electron candidate and its containment within the electromagnetic part of the calorimeter.

\footnotetext{
${ }^{2}$ The scattered positron is referred to as an electron throughout this paper.
} 
An event vertex, reconstructed by the central or forward tracker, is required within $30(35) \mathrm{cm}$ of the nominal interaction point for the SpaCal (LAr) electron samples. To suppress background where a photon fakes the scattered electron, a charged particle track segment must be associated to the electron candidate. This track is reconstructed in the BDC for SpaCal electron candidates and in the central tracking system for LAr electron candidates. The polar angle $\theta_{e}^{\prime}$ of the scattered electron is calculated from the interaction vertex and the BDC track (the LAr cluster) for SpaCal (LAr) electrons. In order to ensure good acceptance for the electron in the calorimeters and associated trackers, the scattered electron polar angle range considered is $156^{\circ}<\theta_{e}^{\prime}<176.5^{\circ}$ for the SpaCal electron samples and $\theta_{e}^{\prime}<153^{\circ}$ for the LAr electron sample.

Hadronic final state objects are reconstructed from the LAr and SpaCal calorimeters and the central tracking system using an energy flow algorithm which combines charged particle tracks with calorimeter deposits without double counting [48]. Isolated low energy calorimeter deposits are classified as noise and are rejected from the analysis. To further suppress backgrounds, a minimum of two remaining hadronic final state objects is demanded. Consistency is required between the variable $y$, as reconstructed according to

$$
\begin{array}{lll}
y_{e}=1-E_{e}^{\prime} / E_{e} \cdot \sin ^{2}\left(\theta_{e}^{\prime} / 2\right) & & \text { (Electron method), } \\
y_{h}=\left(E-p_{z}\right)_{h} / 2 E_{e} & & \text { (Hadron method) }, \\
y_{d}=\tan (\gamma / 2) /\left[\tan \left(\theta_{e}^{\prime} / 2\right)+\tan (\gamma / 2)\right] & & \text { (Double angle method), }
\end{array}
$$

using, respectively, the scattered electron only, the hadronic final state ${ }^{3}$ only [49], and the electron and hadronic final state polar angles $\theta_{e}^{\prime}$ and $\gamma$ [50]. The criteria $\left|y_{e}-y_{h}\right|<0.25$ and $\left|y_{e}-y_{d}\right|<0.25$ remove badly reconstructed events, further suppress photoproduction background and reduce QED radiative corrections due to photon emission from the initial state positron.

The variables $y, Q^{2}$ and $x$ are reconstructed by combining information from the scattered electron and the hadronic final state using the method introduced in [5]:

$$
y=y_{e}^{2}+y_{d}\left(1-y_{d}\right) ; \quad Q^{2}=\frac{4 E_{e}^{2}(1-y)}{\tan ^{2}\left(\theta_{e}^{\prime} / 2\right)} ; \quad x=\frac{Q^{2}}{s y} .
$$

This reconstruction method interpolates between the electron method at large $y$ where it has the best performance and the double angle method at low $y$. For diffractive events, where the $X$ component of the hadronic final state is well contained in the central detectors, the polar angle $\gamma$ is well measured and the double angle method has an improved resolution compared with that for non-diffractive events. This method yields a resolution of $5-15 \%$ in $y$, improving with increasing $y$. The resolution in $Q^{2}$ is around $3 \%$.

The principal selection criterion for the charged current sample is a large missing transverse momentum, corresponding to the unobserved final state neutrino. This is identified at the trigger level mainly using the Liquid Argon calorimeter. The trigger efficiency exceeds $60 \%$ throughout the kinematic range studied here. For the final selection, the missing transverse momentum must exceed $12 \mathrm{GeV}$. A reconstructed event vertex is required as for the neutral current case. To

\footnotetext{
${ }^{3}$ Here and in the following, the four-vector of the reconstructed hadronic final state is denoted $\left(E, p_{x}, p_{y}, p_{z}\right)_{h}$. In diffractive events, $e p \rightarrow e X Y$, where the leading baryonic system $Y$ is not observed, this corresponds to the four-vector of the $X$ system.
} 
suppress backgrounds, further selection criteria are applied on the event topology, as described in [41]. The inclusive kinematic variables are reconstructed using the final state hadrons [49].

The selection $y>0.04$ is applied to all data samples to ensure reasonable containment of the hadronic final state in the central detectors. For the neutral current LAr electron data, the sample is restricted to $y_{e}<0.63$ for $Q^{2}<890 \mathrm{GeV}^{2}$ and to $y_{e}<0.9$ for $Q^{2}>890 \mathrm{GeV}^{2}$, which suppresses photoproduction background.

\subsection{Selection and Reconstruction of Diffractive Events}

Diffractive events are selected on the basis of a large rapidity gap, separating the leading baryonic system $Y$ from the system $X$. The rapidity gap is inferred from the absence of activity in detectors sensitive to forward energy flow. The pseudorapidity of the most forward energy deposit above a noise threshold of $400 \mathrm{MeV}$ in the LAr calorimeter must satisfy $\eta_{\max }<3.3$. This requirement ensures that the forward extent of the $X$ system lies within the acceptance range of the main detector components and thus that $M_{\mathrm{X}}$ can be reconstructed reliably. There must also be no activity above noise thresholds in the Plug, FMD and PRT detectors. Studies of the correlations between the activity levels in the different forward detector components show that the LAr, Plug, FMD and PRT requirements have rejection efficiencies for events with no large rapidity gap of around 95\%, 80\%,80\% and 30\%, respectively. These efficiencies are well described by the simulations of inclusive DIS used in the analysis (section 3.6) and the combined efficiency for the rejection of events with hadronic activity in the range $3.3<\eta \lesssim 7.5$ is close to $100 \%$. Corrections of around $5 \%$, evaluated using randomly triggered events, are applied to account for the component of the diffractive signal which is rejected due to electronic noise, synchrotron radiation or other effects which fake activity in the forward detectors.

The diffractive event kinematics are reconstructed using the mass of the system $X$, obtained from

$$
M_{\mathrm{x}}=\sqrt{\left(E^{2}-p_{x}^{2}-p_{y}^{2}-p_{z}^{2}\right)_{h} \cdot \frac{y}{y_{h}}} .
$$

Neglecting the transverse momentum of the hadrons, this method of reconstructing $M_{\mathrm{X}}$ reduces at large $y$ to a measurement of $\sqrt{2\left(E+p_{z}\right)_{h}\left(E_{e}-E_{e}^{\prime} \sin ^{2} \frac{\theta_{e}^{\prime}}{2}\right)}$, thus improving the resolution where losses of hadrons in the backward $(-z)$ direction become significant. The resolution in $M_{\mathrm{x}}$ varies between $20 \%$ and $30 \%$ in the measured kinematic range. The diffractive variables $\beta$ and $x_{I P}$ are obtained from

$$
\beta=\frac{Q^{2}}{Q^{2}+M_{\mathrm{x}}^{2}} ; \quad x_{\mathbb{P}}=\frac{x}{\beta} .
$$

The sensitivity of the measurement to variations in the details of the selection and reconstruction has been tested in detail. For example, there is no significant change in the results when one or more of the forward detectors is not used in the measurement. The final charged current sample is scanned visually and all events are consistent with production via $e p$ scattering in the interaction region. 


\subsection{Measurement Ranges in $M_{\mathrm{Y}}$ and $t$}

The large rapidity gap selection yields a sample which is dominated by the single dissociation process $e p \rightarrow e X p$, with the proton transverse momentum $p_{t}(p)$, and hence $|t| \simeq p_{t}^{2}(p)$, relatively small. However, there is an admixture of proton dissociative events, $e p \rightarrow e X Y$, where the proton dissociation system has a small mass $M_{\mathrm{Y}}$. The ranges of sensitivity of the measurement in $M_{\mathrm{Y}}$ and $t$ are determined by the acceptances of the forward detectors which are used to identify the large rapidity gap (section 3.4).

In order to keep the uncertainties arising from proton dissociation small and to ease comparisons with previous data $[5,33,34]$, the measurement is integrated over the region

$$
M_{\mathrm{Y}}<1.6 \mathrm{GeV}, \quad|t|<1 \mathrm{GeV}^{2} .
$$

The correction factors applied to account for the net migrations about these limits are determined by evaluation of the forward detector response to elastic proton and proton dissociative processes $^{4}$, using the DIFFVM [51] simulation. The ratio of the full generated proton dissociation cross section to the generated elastic cross section is taken to be 1:1. Proton dissociation is simulated using an approximate $\mathrm{d} \sigma / \mathrm{d} M_{\mathrm{Y}}^{2} \propto 1 / M_{\mathrm{Y}}^{2}$ dependence, with explicit simulations of the most important resonances at low $M_{\mathrm{Y}}$ [52]. The $t$ dependence for proton dissociation follows $\mathrm{d} \sigma / \mathrm{d} t \propto e^{B_{P D} t}$ with a slope parameter $B_{P D}=1 \mathrm{GeV}^{-2}$. The uncertainties are evaluated by varying the details of this simulation as described in section 3.7. The resulting correction factors are $-8.2 \pm 5.8 \%$ ('1997 MB'), $-8.6 \pm 5.8 \%$ ('1997 all') and $-12.0 \pm 7.4 \%$ ('1999-2000').

Comparison of the current data with a similar measurement in which the leading proton is directly measured [6] yields a ratio of cross sections for $M_{\mathrm{Y}}<1.6 \mathrm{GeV}$ and $M_{\mathrm{Y}}=m_{p}$ of $1.23 \pm 0.03$ (stat.) \pm 0.16 (syst.) which is consistent with the DIFFVM prediction of $1.15_{-0.08}^{+0.15}$. Neither the comparison in [6], nor further studies sensitive to the larger $M_{\mathrm{Y}}$ region [45], show any evidence for a dependence of the ratio of proton dissociation to elastic cross sections on any of the measured kinematic variables in the region under study.

\subsection{Simulations and Corrections to the Data}

Corrections for detector inefficiencies, acceptances and migrations between $x_{\mathbb{P}}, \beta$ and $Q^{2}$ measurement intervals are evaluated using a Monte Carlo simulation which combines several models. The RAPGAP [53] event generator simulates the processes $e^{+} p \rightarrow e^{+} X p$ and $e^{+} p \rightarrow \bar{\nu}_{e} X p$ with $x_{\mathbb{P}}<0.15$, assuming proton vertex factorisation. Both pomeron and sub-leading exchanges are included, based on the DPDFs from a leading order QCD fit to previous H1 data ('fit 2' in [5]). The parton densities are evolved using $Q^{2}$ as a scale and are convoluted with leading order QCD matrix elements. Higher order QCD radiation is modelled using initial and final state parton showers in the leading $\log \left(Q^{2}\right)$ approximation [54]. Hadronisation is simulated using the Lund string model [55] as implemented in JETSET [56]. QED radiative effects, including virtual loop corrections, are taken into account via an interface to the HERACLES program [57]. Small weighting factors are applied to the neutral current simulation to ensure

\footnotetext{
${ }^{4}$ Only proton dissociation to low $M_{\mathrm{Y}}$ states is considered in this procedure. Proton dissociation with $M_{\mathrm{Y}}>$ $5 \mathrm{GeV}$ is simulated using the inclusive Monte Carlo models as described in section 3.6.
} 
that the $t$ dependence matches that measured in [6] and to optimise the description of the current data. The DIFFVM model [51] is used to simulate the exclusive production of the $\rho, \omega, \phi$ and $J / \psi$ vector mesons, which contribute significantly in the SpaCal electron samples at small $M_{\mathrm{x}}$ (high $\beta$ ).

Due to the small inefficiency in the rejection of events with forward hadronic activity using the forward detectors, small contributions in the selected data arise from the regions $x_{\mathbb{P}}>0.15$ and $M_{\mathrm{Y}}>5 \mathrm{GeV}$. These backgrounds are subtracted using a simulation based on the DJANGO [58] Monte Carlo model of inclusive DIS for the SpaCal electron sample and the non-diffractive RAPGAP simulation [53] for the LAr electron and charged current samples. The cross sections in the inclusive simulations are obtained from QCD fits to recent H1 DIS data [47,59].

Residual photoproduction background, which is sizeable only at the highest $y$ values, is subtracted on a statistical basis. Its contribution to the data is evaluated using the PHOJET [60] model for the SpaCal electron data and the PYTHIA [56] Monte Carlo model for the charged current sample. For the neutral current LAr data, the background is evaluated from the fraction of reconstructed events for which the detected lepton candidate has the opposite charge to the beam lepton, under the assumption that the background is charge symmetric [41]. The small backgrounds near $\beta=1$ from QED-Compton scattering (ep $\rightarrow e p \gamma)$ and from di-lepton production via photon-photon fusion $\left(e p \rightarrow e p e^{+} e^{-}\right.$) are subtracted using the COMPTON [61] and LPAIR [62] Monte Carlo simulations, respectively. In the charged current measurement, a further small background from the production of real electroweak gauge bosons is simulated using the EPVEC [63] Monte Carlo model. The normalisations and kinematic dependences of each of the background simulations have been checked using dedicated alternative selections designed to enhance the corresponding background.

\subsection{Systematic Uncertainties}

A detailed systematic error analysis has been performed, in which the sensitivity of the measurements to variations in the efficiencies and energy scales of the detector components and to the details of the correction procedure is tested. For the neutral current measurement, the systematic error sources leading to uncertainties which are correlated between data points are listed below.

- The uncertainty on the SpaCal electromagnetic energy scale varies from $2.4 \%$ at $E_{e}=$ $6.5 \mathrm{GeV}$ to $0.5 \%$ at $E_{e}=27.5 \mathrm{GeV}$ [47]. For electrons detected in the LAr calorimeter, the energy scale is known to a precision varying between $1 \%$ and $1.5 \%$, depending on $\theta_{e}^{\prime}$ [41]. The uncertainties in the relative alignment of the different detector components are reflected in possible biases in the electron polar angle measurement at the level of $0.5 \mathrm{mrad}$ for the SpaCal data [47] and between $1 \mathrm{mrad}$ and $2 \mathrm{mrad}$, depending on $\theta_{e}^{\prime}$, for the LAr data [41].

- The hadronic energy scale of the LAr calorimeter is known to $2 \%$ for all samples studied. The uncertainty on the hadronic energy scale of the SpaCal is $7 \%$ and that on the contribution to the hadronic energy measurement from charged particle tracks is $3 \%$. 
- Imperfect treatment of calorimeter noise can result in a bias in the reconstruction of $M_{\mathrm{x}}$. The corresponding uncertainty is evaluated by varying the amount of calorimeter energy classified as noise by $10 \%$. This level of precision is determined by comparing the calorimeter noise subtracted in the data with the Monte Carlo model, which includes a simulation of noise based on randomly triggered events.

- The efficiency with which the FMD registers activity when there is hadronic energy flow in its acceptance region is varied by $5 \%$ in the simulation. For the PRT, this efficiency is varied by $20 \%$. The Plug energy scale is varied by $30 \%$. These levels of uncertainty are obtained by comparison of the present data with the Monte Carlo simulation for samples in which forward detector activity is required to be present rather than absent.

- The model dependence of the acceptance and migration corrections and background subtractions is estimated by varying the details of the Monte Carlo simulation within the limits permitted by the present data. In the RAPGAP simulation of diffraction for the SpaCal electron data, the $x_{\mathbb{P}}$ distribution is reweighted by $\left(1 / x_{\mathbb{P}}\right)^{ \pm 0.05}$, the $\beta$ distribution by $\beta^{ \pm 0.05}$ and $(1-\beta)^{ \pm 0.05}$, the $t$ distribution by $e^{ \pm t}$ and the $Q^{2}$ distribution by $\left(\log Q^{2}\right)^{ \pm 0.2}$. The same systematic shifts are applied for the LAr electron data, except that the powers by which the $x_{\mathbb{P}}, \beta$ and $1-\beta$ distributions are reweighted are increased from 0.05 to 0.1 , reflecting the weaker constraints on those distributions from the data at high $Q^{2}$. The normalisation of the sub-leading meson exchange in RAPGAP is varied by $\pm 25 \%$ and that of the vector meson production simulation (DIFFVM) is varied by $\pm 50 \%$. The uncertainty in the background from high $x_{I P}$ or $M_{\mathrm{Y}}$, as simulated by the DJANGO and inclusive RAPGAP Monte Carlo models, is taken to be $100 \%$. Appropriate variations are also made in the normalisations of the photoproduction, QED-Compton scattering and lepton pair production background simulations.

Several further uncertainties, listed below, affect all data points in an identical manner and are thus considered as normalisation uncertainties.

- The uncertainty on the factor accounting for smearing about the $M_{\mathrm{Y}}$ and $t$ boundaries of the measurement (section 3.5) is 5.8\% for the data taken in 1997 and $7.4 \%$ for the 1999-2000 data. The dominant contribution to this uncertainty arises from variations in the assumed ratio of proton dissociation to elastic proton cross sections in the range 0.5 to 2.0, which is determined from studies with alternative forward detector requirements. Smaller contributions arise from reweighting the $M_{\mathrm{Y}}$ and $t$ distributions of the proton dissociation simulation and propagating the uncertainties on the FMD, PRT and Plug detectors.

- The uncertainty arising from the luminosity measurement is $1.5 \%$ for all samples.

- The correction factor for diffractive events rejected due to noise fluctuations in the forward detectors is varied by $25 \%$, which corresponds to the r.m.s. variation over the different fills of HERA. This leads to normalisation uncertainties at the $1 \%$ level, varying slightly between the different data sets. 
A final class of systematic errors leads to uncertainties which are not taken to be correlated between data points.

- The calculated acceptance of the $\eta_{\max }$ cut depends on the modelling of the hadronic final state topology. The associated uncertainty is estimated from the effect of using an alternative model for higher order QCD processes (the colour dipole approach [64] as implemented in ARIADNE [65] in place of parton showers). This results in an uncertainty which depends to good approximation on $x_{\mathbb{P}}$ only and varies between $1.2 \%$ at $x_{\mathbb{P}}=0.0003$ and $11 \%$ at $x_{\mathbb{P}}=0.03$.

- The uncertainty in the trigger efficiency is $1.0 \%$ in the SpaCal data [47] and $0.3 \%$ for LAr data [41].

- Uncertainties of $0.5 \%$ arise in all neutral current data sets due to the uncertainty in the efficiency of the track-link requirements for the electron candidate [41,47].

The total systematic uncertainty on each data point is formed by adding the individual contributions in quadrature. Away from the boundaries of the kinematic region studied in the neutral current measurement, the systematic error varying from point to point is around $5 \%$, with no single source of uncertainty dominating. The systematic error increases to typically $15 \%$ at the largest $x_{I P}=0.03$, where the contribution from the modelling of the acceptance of the $\eta_{\max }$ requirement becomes important. At small $M_{\mathrm{x}}$ values, the calorimeter noise uncertainty becomes the largest. These point-to-point systematic uncertainties are to be compared with normalisation uncertainties of $6.2 \%$ and $7.6 \%$ for the SpaCal and LAr electron data, respectively, and statistical errors of between $5 \%$ and $20 \%$.

For the charged current measurement, the statistical uncertainties are dominant. The systematic error treatment is similar to that used in the neutral current case, except that the model variations are increased in light of the lack of previous data to constrain the kinematic dependences. The largest systematic uncertainties arise from these model uncertainties, in particular from that on the sub-leading meson exchange contribution, and from the modelling of the acceptance of the $\eta_{\max }$ requirement.

\section{The Diffractive Neutral Current Cross Section}

In order to obtain the reduced neutral current cross section defined in equation 4 , the data are corrected to fixed values of $Q^{2}, \beta$ and $x_{I P}$, the influence of the finite bin sizes being evaluated using a parameterisation of the QCD fit described in section 5. The measurements are quoted at the Born level after the corrections for QED radiative effects described in section 3.6. For all data points shown, the acceptance, bin purity and bin stability ${ }^{5}$ exceed $30 \%$. The results are given in numerical form in tables 4 (SpaCal electron data) and 5 (LAr electron data) and are shown graphically as described in sections 4.1 and 4.2. They can also be found at [66].

\footnotetext{
${ }^{5}$ Purity (stability) is evaluated using the Monte Carlo simulation and is defined as the fraction of events reconstructed (generated) in a measurement bin which are also generated (reconstructed) in that bin.
} 


\subsection{Dependences on $\beta$ and $Q^{2}$}

The QCD properties and structure of the diffractive interaction are most easily interpreted from the dependences on $x$ (or equivalently $\beta$ ) and $Q^{2}$, with $x_{I P}$ fixed. According to [20], DPDFs can then be defined for each fixed $x_{\mathbb{P}}$ value, independently of the validity of proton vertex factorisation. A binning scheme is therefore adopted with fixed $x_{I P}, x$ and $Q^{2}$ values. In order to minimise the statistical uncertainties and the systematic uncertainties associated with the reconstruction of $M_{\mathrm{x}}$, relatively large $x_{\mathbb{P}}$ intervals are chosen. The binning in the much better resolved variables, $x$ and $Q^{2}$, is chosen to match previous inclusive measurements [47].

The $\beta$ and $Q^{2}$ dependences of the reduced cross section, multiplied by $x_{\mathbb{P}}$, are shown in figures $2-6$ at fixed values of $x_{P P}=0.0003,0.001,0.003,0.01$ and 0.03 , respectively. The results from different $x_{\mathbb{P}}$ values complement one another in their $\beta$ and $Q^{2}$ ranges, though there is also considerable overlap between the coverage at different $x_{\mathbb{P}}$ values. For each $x_{\mathbb{P}}$ value considered, the data exhibit similar $\beta$ and $Q^{2}$ dependences.

As can be seen in figures $2 \mathrm{a}-6 \mathrm{a}$, the cross section remains large up to the highest accessed values of $\beta$ (i.e. where $x \rightarrow x_{\mathbb{P}}$ ) at fixed $x_{\mathbb{P}}$ and $Q^{2}$. This behaviour is in marked contrast to that of hadron structure functions, but is qualitatively similar to that of the photon [67]. The $Q^{2}$ dependence of the data is shown for fixed $x_{\mathbb{P}}$ and $\beta$ in figures $2 \mathrm{~b}-6 \mathrm{~b}$. The reduced cross section increases with $Q^{2}$ throughout most of the kinematic range, up to large $\beta \simeq 0.5$. These positive scaling violations confirm earlier observations [5] and contrast with the case of inclusive scattering from hadrons, for which the cross section at fixed $x$ falls with increasing $Q^{2}$ for $x \gtrsim 0.1$ [47].

The data in figures 2-6 are compared ${ }^{6}$ with the results of the 'H1 2006 DPDF Fit A' described in section 5. This fit assumes proton vertex factorisation and includes a sub-leading exchange contribution, which is important at low $\beta$ and large $x_{\mathbb{P}}$ as shown in figures 5a and 6a. It is clear from the good overall description that the data are broadly consistent with such a model. The $\beta$ dependence of $\sigma_{r}^{D(3)}$ then directly reflects the quark structure of the diffractive exchange with each quark flavour weighted by its squared electric charge, whilst the measured $Q^{2}$ dependence is sensitive to the diffractive gluon density.

The $Q^{2}$ dependence is quantified by fitting the data at fixed $x_{\mathbb{P}}$ and $\beta$ to the form

$$
\sigma_{r}^{D(3)}\left(x_{\mathbb{P}}, Q^{2}, \beta\right)=a_{\mathrm{D}}\left(\beta, x_{\mathbb{P}}\right)+b_{\mathrm{D}}\left(\beta, x_{\mathbb{P}}\right) \ln Q^{2},
$$

such that $b_{\mathrm{D}}\left(\beta, x_{\mathbb{P}}\right)=\left[\partial \sigma_{r}^{D(3)} / \partial \ln Q^{2}\right]_{\beta, x_{\mathbb{P}}}$ is the first logarithmic $Q^{2}$ derivative of the reduced cross section. This observable has been used previously to discriminate between different models of diffractive DIS [68]. Equation 10 is fitted to data with $0.001 \leq x_{\mathbb{P}} \leq 0.03$ from each $\beta$ value if there are a minimum of three available data points ${ }^{7}$. The resulting $\ln Q^{2}$ derivatives are shown in figure $7 \mathrm{a}$, after dividing $b_{\mathrm{D}}\left(\beta, x_{\mathbb{P}}\right)$ by the factor $f_{\mathbb{P} / p}\left(x_{\mathbb{P}}\right)$, defined in equation 14 , which is used to parameterise the $x_{\mathbb{P}}$ dependence so that the results from different $x_{\mathbb{P}}$ values

\footnotetext{
${ }^{6}$ The curves shown correspond to $E_{p}=820 \mathrm{GeV}$. The predictions for $E_{p}=920 \mathrm{GeV}$ differ slightly at the lowest $\beta$ values, due to the influence of $F_{L}^{D(3)}$.

${ }^{7}$ If only data with $Q^{2} \geq 8.5 \mathrm{GeV}^{2}$ are included, as in the fit described in section 5, the changes to the logarithmic $Q^{2}$ derivatives are small and the conclusions are unaffected.
} 
can be compared in normalisation as well as in shape. Although the logarithmic derivatives at different $x_{I P}$ values probe different $Q^{2}$ regions, they are remarkably similar when viewed as a function of $\beta$. This confirms the applicability of the proton vertex factorisation framework to the description of the current data. The lack of any significant change in behaviour at large $x_{I P}$ indicates that the derivatives are not significantly affected by the presence of sub-leading exchange contributions.

According to the DGLAP evolution equations, the $\ln Q^{2}$ derivative of $F_{2}^{D(3)}$ contains contributions due to the splittings $g \rightarrow q \bar{q}$ and $q \rightarrow q g$, convoluted with the diffractive gluon and quark densities, respectively. In figure $7 \mathrm{~b}$, the derivatives at $x_{\mathbb{P}}=0.01$ are shown together with the decomposition into these two contributions according to the 'H1 2006 DPDF Fit A' described in section 5. The curves correspond to $Q^{2}$ values which vary in order to match the average $\ln Q^{2}$ of the data which are fitted at each $\beta$ value. The theoretical calculation is in good agreement with the data. The $\ln Q^{2}$ derivative is determined almost entirely by the diffractive gluon density up to $\beta \simeq 0.3$. The large positive $\ln Q^{2}$ derivatives in this region can thus be attributed to a large gluonic component in the DPDFs. For $\beta \gtrsim 0.3$, the contribution to the $Q^{2}$ evolution from quark splittings becomes increasingly important and the derivatives become less sensitive to the gluon density. The fall in the derivative as $\beta \rightarrow 1$ then arises dominantly from gluon radiation, $q \rightarrow q g$, shifting quarks from higher to lower $\beta$ with increasing $Q^{2}$.

Considering diffractive DIS in terms of the elastic scattering from the proton of colour dipoles formed by partonic fluctuations of the exchanged virtual photon [69] provides a complementary framework in which to describe diffractive DIS. Modelling the exchange by two gluons in a net colour singlet configuration [70], the data at low and moderate $\beta$ are then described in terms of $q \bar{q}$ and $q \bar{q} g$ fluctuations of transversely polarised photons, whilst the high $\beta$ region contains a $Q^{2}$-suppressed non-leading twist contribution from $q \bar{q}$ fluctuations of longitudinally polarised photons $[17,18,71]$. A significant contribution from this term could explain the tendency of the data to rise with increasing $\beta$ for $\beta>0.1$ at low $Q^{2}$ (figure $2 \mathrm{a}-4 \mathrm{a}$ ). However, the high $\beta$ data in figures $2 \mathrm{~b}-6 \mathrm{~b}$ are adequately described by a single logarithmic dependence on $Q^{2}$ and do not require a sum of leading and $Q^{2}$-suppressed terms.

\subsection{Dependence on $x_{\mathbb{P}}$ and Comparisons with other Data}

For comparison with previous measurements of diffractive DIS and for a more detailed study of the $x_{\mathbb{P}}$ dependence at fixed $\beta$ and $Q^{2}$, the reduced cross section is also measured using a binning scheme with fixed $\beta, Q^{2}$ and $x=\beta \cdot x_{\mathbb{P}}$, as shown in figure 8 . The data are multiplied by $x_{\mathbb{P}}$ for visibility and are compared with the results of the QCD fit described in section 5, which is also in good agreement with the data obtained using this binning scheme. The $x_{I P}$ dependence is roughly flat for all $\beta$ and $Q^{2}$ values, implying that the reduced cross section approximately follows a $\sigma_{r}^{D(3)} \propto 1 / x_{\mathbb{P}}$ dependence. However, when viewed in detail, there are clear deviations from this behaviour. The variations in the $x_{\mathbb{P}}$ dependence as $\beta$ changes are as expected from the interplay between the leading pomeron and a sub-leading trajectory exchange, as discussed in section 5.4.

In figure 9, the measured reduced cross section is compared with results obtained by direct measurement of the final state proton using the H1 Forward Proton Spectrometer ('H1 
(FPS)') [6] and the ZEUS Leading Proton Spectrometer ('ZEUS (LPS)') [10]. In figure 10, a comparison is made with ZEUS data obtained by decomposition of the inclusive $\ln M_{\mathrm{x}}^{2}$ distribution into diffractive and non-diffractive components ('ZEUS $\left.\left(M_{\mathrm{x}}\right)^{\prime}\right)$ [11]. Shifts, evaluated using the DPDF fit described in section 5, are applied to these data in order to transport them to the $\beta$ and $Q^{2}$ values of the present measurement. Since no uncertainties are ascribed to this procedure, only data points for which the shifts are small and relatively insensitive to the choice of DPDFs are shown. The different contributions from proton dissociation in the different data sets are accounted for by the application of global factors of 1.23 to the 'H1 (FPS)' and 'ZEUS (LPS)' data and of 0.86 to the 'ZEUS $\left(M_{\mathrm{x}}\right)$ ' data. The former factor corresponds to the measured ratio of cross sections for $M_{\mathrm{Y}}<1.6 \mathrm{GeV}$ and $M_{\mathrm{Y}}=m_{p}$, for which the uncertainty is 0.16 (see [6] and section 3.5). The latter factor contains an additional contribution of 0.7 , corresponding to the ratio of cross sections for $M_{\mathrm{Y}}=m_{p}$ and $M_{\mathrm{Y}}<2.3 \mathrm{GeV}$ according to [11].

There is broad agreement between all of the data sets on the general behaviour of the diffractive cross section. The 'H1 (FPS)' and 'ZEUS (LPS)' data are compatible with the present measurement throughout the kinematic range available for comparison. A more detailed comparison with the 'H1 (FPS)' data is presented in [6]. The 'ZEUS $\left(M_{\mathrm{x}}\right)$ ' data are in good agreement with the present measurement in some regions, for example at low $\beta$ and low $Q^{2}$. However, there are disagreements in the low $Q^{2}$, high $\beta$ region and in the high $Q^{2}$, low $\beta$ region, which correspond to low and high values of $M_{\mathrm{x}}=\sqrt{Q^{2}(1 / \beta-1)}$, respectively. The high $M_{\mathrm{x}}$ discrepancy gives rise to a weaker $Q^{2}$ dependence of the 'ZEUS $\left(M_{\mathrm{x}}\right)$ ' cross section at low $\beta$ than is the case for H1 [72].

\section{QCD Analysis and Diffractive Parton Distributions}

The high precision and large kinematic range of the diffractive cross section data presented in this paper allow detailed tests of the factorisation properties of diffractive DIS and the extraction of DPDFs, which may be used to predict cross sections for other diffractive processes at HERA and elsewhere.

\subsection{Theoretical Framework}

QCD hard scattering collinear factorisation, when applied to diffractive DIS [20], implies that the cross section for the process $e p \rightarrow e X Y$ can be written in terms of convolutions of partonic cross sections $\hat{\sigma}^{e i}\left(x, Q^{2}\right)$ with DPDFs $f_{i}^{D}$ as

$$
\mathrm{d} \sigma^{e p \rightarrow e X Y}\left(x, Q^{2}, x_{\mathbb{P}}, t\right)=\sum_{i} f_{i}^{D}\left(x, Q^{2}, x_{\mathbb{P}}, t\right) \otimes \mathrm{d} \hat{\sigma}^{e i}\left(x, Q^{2}\right) .
$$

The partonic cross sections are the same as those for inclusive DIS. The DPDFs represent probability distributions for partons $i$ in the proton under the constraint that the proton is scattered to a particular system $Y$ with a specified four-momentum. They are not known from first principles, but can be determined from fits to the data using the DGLAP [39] evolution equations. The factorisation formula in equation 11 is valid for sufficiently large $Q^{2}$ and fixed $x_{\mathbb{P}}, t$ and 
system $Y$. It also applies to any cross section which is integrated over a fixed range in $M_{\mathrm{Y}}$ and $t$ and may thus be applied to the present data with $M_{\mathrm{Y}}<1.6 \mathrm{GeV}$ and $|t|<1 \mathrm{GeV}^{2}$.

Due to kinematic constraints, it is not possible to access the full range of $x$ and $Q^{2}$ using data from only one value of $x_{\mathbb{P}}$. A parameterisation of the $x_{\mathbb{P}}$ dependence of the DPDFs, for which there is no clear procedure in $\mathrm{QCD}$, is therefore necessary. The proton vertex factorisation framework is adopted here, such that the DPDFs are factorised into a term depending only on $x_{\mathbb{P}}$ and $t$ and a term depending only on $x\left(\right.$ or $\beta$ ) and $Q^{2}$ :

$$
f_{i}^{D}\left(x, Q^{2}, x_{\mathbb{P}}, t\right)=f_{\mathbb{P} / p}\left(x_{\mathbb{P}}, t\right) \cdot f_{i}\left(\beta=x / x_{\mathbb{P}}, Q^{2}\right) .
$$

This is equivalent to treating the diffractive exchange as a 'pomeron' with a partonic structure given by the parton distributions $f_{i}\left(\beta, Q^{2}\right)$, the variable $\beta$ corresponding to the fraction of the pomeron longitudinal momentum carried by the struck quark. The 'pomeron flux factor' $f_{\mathbb{P} / p}\left(x_{\mathbb{P}}, t\right)$ represents the probability that a pomeron with particular values of $x_{\mathbb{P}}$ and $t$ couples to the proton. In the low $x_{\mathbb{P}}$ region where sub-leading exchange contributions are negligible, the data presented in section 4 are consistent with factorisation of the $x_{\mathbb{P}}$ dependence, as required for the proton vertex factorisation expressed in equation 12. There is also no evidence from the investigations in $[6,10]$ for any change in the $t$ or $M_{\mathrm{Y}}$ dependences as either $\beta$ or $Q^{2}$ vary in the range relevant to the present analysis.

\subsection{Fit Procedure}

To determine the DPDFs, fits are made to the reduced cross section data as presented in figures $2-6$, for which $\beta \leq 0.8$. In order to avoid regions which are most likely to be influenced by higher twist contributions or other problems with the chosen theoretical framework, only data with $M_{\mathrm{x}}>2 \mathrm{GeV}$ are included in the fit and the region $Q^{2}<8.5 \mathrm{GeV}^{2}$ is excluded, as explained in section 5.3. The total number of fitted data points is 190 .

Input parameters describing the DPDFs at a starting scale $Q_{0}^{2}$ for QCD evolution are adjusted to obtain the best description of the data after NLO DGLAP [40] evolution ${ }^{8}$ to $Q^{2}>Q_{0}^{2}$ and convolution of the DPDFs with coefficient functions. The fit is performed in the $\overline{M S}$ renormalisation scheme [74] with charm and beauty quarks treated as massive, appearing via boson gluon fusion-type processes up to order $\alpha_{s}^{2}$ [75]. The heavy quark masses are set to world average values (see table 2). The strong coupling is set via $\Lambda_{Q C D}^{(3)}=399 \pm 37 \mathrm{MeV}$ for 3 flavours, which corresponds [76] to the world average $\alpha_{s}^{(5)}\left(M_{Z}^{2}\right)$ for five flavours (table 2). The effects of $F_{L}^{D(3)}$ are considered through its relation to the quark and gluon densities at NLO. Since the relative normalisations of the diffractive parton densities and the flux factor in equation 12 are arbitrary, no momentum sum rule is imposed.

The DPDFs are modelled in terms of a light flavour singlet distribution $\Sigma(z)$, consisting of $u, d$ and $s$ quarks and anti-quarks with $u=d=s=\bar{u}=\bar{d}=\bar{s}$, and a gluon distribution $g(z)$. Here, $z$ is the longitudinal momentum fraction of the parton entering the hard sub-process with respect to the diffractive exchange, such that $z=\beta$ for the lowest order quark-parton

\footnotetext{
${ }^{8}$ Direct pomeron to parton splitting functions, leading to an inhomogeneous term in the DGLAP evolution equations [32,73], are not considered. The presence of such a term would lead to a reduced gluon density.
} 
model process, whereas $0<\beta<z$ for higher order processes. The quark singlet and gluon distributions are parameterised at $Q_{0}^{2}$ using a similar approach to that commonly applied to hadronic parton densities [77-79], such that the most general form is

$$
z f_{i}\left(z, Q_{0}^{2}\right)=A_{i} z^{B_{i}}(1-z)^{C_{i}} .
$$

The exact choices of terms included for the quark singlet and gluon densities and the parameterisation scale $Q_{0}^{2}$ are determined through a systematic investigation of the parameter space, as described in section 5.3. The DPDFs as defined in equation 13 are multiplied by a term $e^{-\frac{0.01}{1-z}}$ in order to ensure that they vanish at $z=1$, as required for the evolution equations to be solvable. The parameters $C_{q}$ and $C_{g}$ thus have the freedom to take negative as well as positive values. Modifying the argument of the exponential term within reasonable limits has no visible influence on the fit quality or the extracted DPDFs in the range of the measurement.

The $x_{\mathbb{P}}$ dependence is parameterised using a flux factor motivated by Regge theory,

$$
f_{\mathbb{P} / p}\left(x_{\mathbb{P}}, t\right)=A_{\mathbb{P}} \cdot \frac{e^{B_{\mathbb{P}} t}}{x_{\mathbb{P}}^{2 \alpha_{\mathbb{P}}(t)-1}},
$$

where the pomeron trajectory is assumed to be linear, $\alpha_{\mathbb{P}}(t)=\alpha_{\mathbb{P}}(0)+\alpha_{\mathbb{P}}^{\prime} t$, and the parameters $B_{\mathbb{P}}$ and $\alpha_{\mathbb{P}}^{\prime}$ and their uncertainties are obtained from fits to H1 FPS data [6]. The values of these and other parameters which are fixed in the fits are summarised in table 2. Following the convention of [5], the value of the normalisation parameter $A_{\mathbb{P}}$ is chosen such that $x_{\mathbb{P}} \cdot \int_{t_{\text {cut }}}^{t_{\min }} f_{\mathbb{P} / p} \mathrm{~d} t=1$ at $x_{\mathbb{P}}=0.003$, where $\left|t_{\min }\right| \simeq m_{p}^{2} x_{\mathbb{P}}^{2} /\left(1-x_{\mathbb{P}}\right)$ is the minimum kinematically accessible value of $|t|, m_{p}$ is the proton mass and $\left|t_{\text {cut }}\right|=1.0 \mathrm{GeV}^{2}$ is the limit of the measurement.

\begin{tabular}{|c|c|c|}
\hline Parameter & Value & Source \\
\hline \hline$\alpha_{\mathbb{P}}^{\prime}$ & $0.06_{-0.06}^{+0.19} \mathrm{GeV}^{-2}$ & {$[6]$} \\
$B_{\mathbb{P}}$ & $5.5_{+0.7}^{-2.0} \mathrm{GeV}^{-2}$ & {$[6]$} \\
$\alpha_{\mathbb{R}}(0)$ & $0.50 \pm 0.10$ & {$[5]$} \\
$\alpha_{\mathbb{R}}^{\prime}$ & $0.3_{-0.3}^{+0.6} \mathrm{GeV}^{-2}$ & {$[6]$} \\
$B_{\mathbb{R}}$ & $1.6_{+0.4}^{-1.6} \mathrm{GeV}^{-2}$ & {$[6]$} \\
$m_{c}$ & $1.4 \pm 0.2 \mathrm{GeV}$ & {$[52]$} \\
$m_{b}$ & $4.5 \pm 0.5 \mathrm{GeV}$ & {$[52]$} \\
$\alpha_{s}^{(5)}\left(M_{Z}^{2}\right)$ & $0.118 \pm 0.002$ & {$[52]$} \\
\hline
\end{tabular}

Table 2: The values of the fixed parameters and their uncertainties, as used in the QCD fits. Since they are strongly anti-correlated when extracted from fits to the FPS data, $\alpha_{\mathbb{P}}^{\prime}$ and $B_{\mathbb{I P}}$ are varied simultaneously to obtain the theoretical errors on the fit results, as are $\alpha_{\mathbb{R}}^{\prime}$ and $B_{\mathbb{R}}$. The remaining parameters are varied independently. The theoretical uncertainties on the free parameters of the fit also contain a contribution from variation of the parameterisation scale $Q_{0}^{2}$, as described in section 5.3.

To obtain a good description of the data, an additional sub-leading exchange $(\mathbb{R})$ is included, which has a lower trajectory intercept than the pomeron and which contributes significantly only 
at low $\beta$ and large $x_{\mathbb{P}}$. As in $[5,6]$ this contribution is assumed to factorise in the same way as the pomeron term, such that equation 12 is modified to

$$
f_{i}^{D}\left(x, Q^{2}, x_{\mathbb{P}}, t\right)=f_{\mathbb{P} / p}\left(x_{\mathbb{P}}, t\right) \cdot f_{i}\left(\beta, Q^{2}\right)+n_{\mathbb{R}} \cdot f_{\mathbb{R} / p}\left(x_{\mathbb{P}}, t\right) \cdot f_{i}^{\mathbb{R}}\left(\beta, Q^{2}\right) .
$$

The flux factor $f_{\mathbb{R} / p}$ takes the form of equation 14, normalised via a parameter $A_{\mathbb{R}}$ in the same manner as for the pomeron contribution and with fixed parameters $\alpha_{\mathbb{R}}(0), \alpha_{\mathbb{R}}^{\prime}$ and $B_{\mathbb{R}}$ obtained from other $\mathrm{H} 1$ measurements (see table 2). The parton densities $f_{i}^{\mathbb{R}}$ of the sub-leading exchange are taken from a parameterisation derived from fits to pion structure function data [80]. Choosing a different parameterisation [81] does not affect the fit results significantly.

The free parameters of the fit are the $A, B$ and $C$ parameters which determine the quark singlet and gluon distributions (equation 13), together with $\alpha_{\mathbb{P}}(0)$, which controls the $x_{\mathbb{P}}$ dependence and $n_{\mathbb{R}}$, which controls the normalisation of the sub-leading exchange contribution. In order to constrain these parameters, a $\chi^{2}$ function as defined in [47] is minimised. This function involves the combined statistical and uncorrelated systematic errors for each data point and also takes account of correlations between data points caused by systematic uncertainties by allowing variations in each systematic error source at the expense of increases in the $\chi^{2}$ variable. Ten correlated systematic error parameters are considered for each of the SpaCal and LAr electron data sets, one for each of the error sources listed in tables 4 and 5 . In this procedure, the sources of correlated uncertainty are taken to be common for the '1997 MB' and '1997 all' data, whereas it is assumed that there are no correlations between the uncertainties on the SpaCal and the LAr electron data. A further systematic error parameter controls the relative normalisation of the LAr electron data set with respect to the SpaCal electron data, for which the uncertainty is $9.8 \%$ (section 3.7). The central results for the DPDFs and other parameters obtained from the fit are not altered significantly if all systematic uncertainties leading to correlations between the data points are ignored.

The statistical and experimental systematic errors on the data points and their correlations are propagated [82] to obtain experimental uncertainties on the DPDFs and other fit parameters, which correspond to increases in the $\chi^{2}$ variable by one unit. The theoretical error is obtained from variations of the assumed parameters as given in table 2, with an additional contribution expressing the sensitivity to the choice of DPDF parameterisation, obtained by varying $Q_{0}^{2}$ as discussed in section 5.3. Since the pomeron flux factor is constrained simultaneously with the parton densities, the possible influence of interference between the pomeron and sub-leading exchange contributions cannot be assessed. However, in previous similar fits in which $\alpha_{\mathbb{P}}(0)$ was extracted separately from the parton densities [5], $\alpha_{\mathbb{P}}(0)$ changed by less than 0.01 between the cases of no interference and maximum constructive interference.

\subsection{Choices of Fit Parameterisation and Kinematic Range}

In order to optimise the results of the fit, the sensitivity to variations in the details of the parameterisation is investigated. With the small numbers of parameters used to describe the parton densities, the $\chi^{2}$ values and the results of the fits are sensitive to the choice of the parameterisation scale $Q_{0}^{2}$ [47], so that its value must be optimised by $\chi^{2}$ minimisation for each parameterisation choice. The $Q_{0}^{2}$-optimised results are then compared in order to make the final parameterisation choice. To ensure that the results of the fit are not sensitive to the kinematic range of the 
data included in the fit, the sensitivity of this procedure to variations in the kinematic cuts is also tested. All parton density parameterisation changes and kinematic range variations lead to extracted values of $\alpha_{\mathbb{P}}(0)$ which are within the experimental uncertainties (see also section 5.4).

The only significant sensitivity to the boundaries of the chosen kinematic range occurs when the minimum $Q^{2}$ value of the data included in the fit, $Q_{\min }^{2}$, is varied. Whereas the quark distribution remains stable within uncertainties for all $Q_{\min }^{2}$ choices, the gluon distribution for $z \lesssim 0.5$ increases systematically as $Q_{\text {min }}^{2}$ varies between $3.5 \mathrm{GeV}^{2}$ and $8.5 \mathrm{GeV}^{2}$, changing by about $40 \%$ in total. The $\chi^{2}$ per degree of freedom also improves steadily as $Q_{\min }^{2}$ varies over this range. There is no evidence for any further variation in the gluon density for $Q_{\min }^{2}>8.5 \mathrm{GeV}^{2}$. The lowest $Q^{2}$ data are therefore omitted from the fit and $Q_{\min }^{2}=8.5 \mathrm{GeV}^{2}$ is chosen. The $Q_{\min }^{2}$ dependence is reflected in figures 2-6 as a tendency for the fit result extrapolated to $Q^{2}<$ $8.5 \mathrm{GeV}^{2}$ to lie below the data. The dependence of the gluon density on $Q_{\min }^{2}$ may indicate inadequacies in the adopted formalism at the lowest $Q^{2}$ values.

The fit results are not sensitive to variations in the minimum or maximum $\beta$ values of the data included, although the minimum $\beta$ is correlated with $Q_{\min }^{2}$ through the kinematics. There is similarly no significant change in the fit results when the minimum $M_{\mathrm{x}}$ value of the data included is increased or when the highest $x_{\mathbb{P}}$ data are omitted.

For the quark singlet distribution, the data require the inclusion of all three parameters $A_{q}$, $B_{q}$ and $C_{q}$ in equation 13 . By comparison, the gluon density is weakly constrained by the data, which are found to be insensitive to the $B_{g}$ parameter. The gluon density is thus parameterised at $Q_{0}^{2}$ using only the $A_{g}$ and $C_{g}$ parameters. With this parameterisation, a value of $Q_{0}^{2}=1.75 \mathrm{GeV}^{2}$ yields the minimum $\chi^{2}$ value of 158 for 183 degrees of freedom. This fit is referred to as the 'H1 2006 DPDF Fit A' in the figures. As a measure of the parameterisation uncertainty, $Q_{0}^{2}$ is varied between $1.15 \mathrm{GeV}^{2}$ and $2.05 \mathrm{GeV}^{2}$, for which the $\chi^{2}$ variable increases by one unit. The correlated systematic error parameters have a mean close to zero and the largest shift in a correlated error source is $1.0 \sigma$. The fit shifts the normalisation of the LAr data relative to the SpaCal data by $0.45 \sigma$. Both the DPDFs and the $\chi^{2}$ per degree of freedom of this fit can be reproduced closely using the approach based on Chebyshev polynomials in [5].

As discussed in section 4.1 (figure $7 \mathrm{~b}$ ), the $Q^{2}$ dependence of the data at fixed $\beta$ and $x_{\mathbb{P}}$ determines the gluon density well at low $\beta$. However, as $\beta$ increases the $\ln Q^{2}$ derivative becomes smaller and the fractional error on the gluon density becomes correspondingly larger. At the highest $\beta$ values, where the $Q^{2}$ evolution is driven by quarks, the $Q^{2}$ dependence of $\sigma_{r}^{D(3)}$ becomes insensitive to the gluon density. The results for the gluon density at large $z$ are thus determined principally by the data at lower $z$ coupled with the parameterisation choice. This lack of sensitivity is confirmed by repeating the fit with the parameter $C_{g}$, which determines the high $z$ behaviour, set to zero. Apart from the exponential term, the gluon density is then a simple constant at the starting scale for evolution, which is chosen to be $Q_{0}^{2}=2.5 \mathrm{GeV}^{2}$ by $\chi^{2}$ minimisation. Even with this very simple parameterisation of the gluon density, the $\chi^{2}$ variable increases only slightly to $\chi^{2}=164$, with 184 degrees of freedom. This fit is referred to as the 'H1 2006 DPDF Fit B' in the figures.

\subsection{Diffractive Parton Distributions and Effective Pomeron Intercept}

A good description of the data is obtained throughout the fitted range $Q^{2} \geq 8.5 \mathrm{GeV}^{2}, \beta \leq 0.8$ and $M_{\mathrm{x}}>2 \mathrm{GeV}$ by both H1 2006 DPDF Fit A and Fit B. The results of Fit A are compared 
with the measured reduced cross section in figures 2-6. The results for the fit parameters are given in table 3 . They can also be found together with the correlation coefficients between the parameters at [66].

\begin{tabular}{|c|c|c|}
\hline Fit Parameter & Fit A & Fit B \\
\hline \hline$\alpha_{\mathbb{P}}(0)$ & $1.118 \pm 0.008$ & $1.111 \pm 0.007$ \\
$n_{\mathbb{R}}$ & $(1.7 \pm 0.4) \times 10^{-3}$ & $(1.4 \pm 0.4) \times 10^{-3}$ \\
$A_{q}$ & $1.06 \pm 0.32$ & $0.70 \pm 0.11$ \\
$B_{q}$ & $2.30 \pm 0.36$ & $1.50 \pm 0.12$ \\
$C_{q}$ & $0.57 \pm 0.15$ & $0.45 \pm 0.09$ \\
$A_{g}$ & $0.15 \pm 0.03$ & $0.37 \pm 0.02$ \\
$C_{g}$ & $-0.95 \pm 0.20$ & 0 (fixed) \\
\hline
\end{tabular}

Table 3: The central values of the parameters extracted in the 'H1 2006 DPDF Fit A' and 'B', and the corresponding experimental uncertainties.

The diffractive quark singlet and gluon distributions from Fit A are shown together with their uncertainties on a logarithmic $z$ scale in figure 11. In order to illustrate the high $z$ region in more detail, they are also shown on a linear $z$ scale in figure 12, where they are compared with the results from Fit B. At low $Q^{2}$, both the quark singlet and the gluon densities remain large up to the highest $z$ values accessed. The quark singlet distribution is well constrained, with an uncertainty of typically $5-10 \%$ and good agreement between the results of Fit A and Fit B. The gluon distribution has a larger uncertainty of typically $15 \%$ at low to moderate $z$ and low $Q^{2}$, dominated by the influence of the $Q_{0}^{2}$ variation. For $z \gtrsim 0.5$, where the sensitivity to the gluon density becomes poor, the level of agreement between Fit A and Fit B worsens.

As shown in figure 13, the fraction of the exchanged momentum carried by gluons integrated over the range $0.0043<z<0.8$, corresponding approximately to that of the measurement, is around $70 \%$ throughout the $Q^{2}$ range studied, confirming the conclusion from earlier work $[5,27]$. The integrated gluon fraction is somewhat smaller for Fit B due to the lower gluon density at large $z$ values, though the results from the two fits are consistent within the uncertainties.

Fit A yields an effective pomeron intercept of

$$
\alpha_{\mathbb{P}}(0)=1.118 \pm 0.008 \text { (exp.) }{ }_{-0.010}^{+0.029}(\text { model }),
$$

where the first error is the full experimental uncertainty and the second expresses the model dependence. This model dependence uncertainty arises dominantly from the variation of $\alpha_{\mathrm{P}}^{\prime}$, which is strongly positively correlated with $\alpha_{\mathbb{P}}(0)$, such that $\alpha_{\mathbb{P}}(0)$ increases to around 1.15 if $\alpha_{\mathbb{P}}^{\prime}$ is set to 0.25 . The intercept has also been shown to be strongly sensitive to the value of $F_{L}^{D}$ [83], though with the NLO treatment adopted here, $F_{L}^{D}$ is determined in the fit and no additional uncertainty is included. The influence of $F_{L}^{D}$ on the reduced cross section according to the fit is shown for $x_{\mathbb{P}}=0.003$ in figure $4 \mathrm{a}$. It is similar at other values of $x_{\mathbb{P}}$. 
The extracted $\alpha_{\mathbb{P}}(0)$ is slightly higher than the value $\alpha_{\mathbb{P}}(0) \simeq 1.08$ expected for the 'soft' pomeron [84] describing long distance hadronic interactions. The result is compatible with that obtained from ZEUS $F_{2}^{D}$ data for $Q^{2} \lesssim 20 \mathrm{GeV}^{2}$ [11]. However, in [11], evidence was reported for an increase of $\alpha_{\mathbb{P}}(0)$ for $Q^{2} \gtrsim 20 \mathrm{GeV}^{2}$. In some models [17, 18], a $\beta$ dependent $\alpha_{\mathbb{P}}(0)$ has also been suggested. Any such dependence of $\alpha_{\mathbb{P}}(0)$ on $Q^{2}$ or $\beta$ implies a breakdown of proton vertex factorisation. In order to test for such effects in the present data, the QCD fit is repeated with additional free parameters corresponding to independent values of $\alpha_{\mathbb{P}}(0)$ in different ranges of $Q^{2}$ or $\beta$. As can be seen from the results in figure 14, there is no evidence for any variation of $\alpha_{\mathbb{P}}(0)$ with either variable within the kinematic range of the fit. This remains the case if the data with $Q^{2}<8.5 \mathrm{GeV}^{2}$ are included. This lack of $Q^{2}$ dependence of $\alpha_{\mathbb{P}}(0)$ contrasts with the $Q^{2}$ dependent effective pomeron intercept extracted in a Regge approach to inclusive small $x$ proton structure function data, as discussed further in section 7 .

The presence of the sub-leading exchange term is required by the data, the $\chi^{2}$ increasing by approximately 40 units if only the pomeron contribution is included. Its relative size is expressed through the normalisation parameter $n_{\mathbb{R}}=\left[1.7 \pm 0.4\right.$ (exp.) ${ }_{-0.8}^{+1.5}($ model $\left.)\right] \times 10^{-3}$ (Fit A), where the dominant uncertainty arises from the correlation with $\alpha_{\mathbb{R}}(0)$. The sub-leading exchange plays a significant role at high $x_{\mathbb{P}}$ and low $\beta$, as shown in figures 5a and 6a. It accounts for around $30 \%(10 \%)$ of the cross section at $x_{\mathbb{P}}=0.03(0.01)$ and is negligible at lower $x_{\mathbb{P}}$.

\section{The Diffractive Charged Current Cross Section}

The diffractive charged current process $e^{+} p \rightarrow \bar{\nu}_{e} X Y$ is sensitive to the diffractive $d, \bar{u}, s$ and $\bar{c}$ densities at large scales. Assuming factorisation, the measurement of the charged current cross section thus tests the assumed flavour decomposition of the quark singlet component of the DPDFs, which is completely unconstrained by the neutral current data. The total charged current cross section integrated over the range $Q^{2}>200 \mathrm{GeV}^{2}, y<0.9$ and $x_{\mathbb{P}}<0.05$ at $\sqrt{s}=319 \mathrm{GeV}$ is measured to be

$$
\sigma_{\mathrm{CC}}^{\text {diff }}=390 \pm 120 \text { (stat.) } \pm 70 \text { (syst.) fb },
$$

corresponding to $2.2 \pm 0.7$ (stat.) \pm 0.4 (syst.) $\%$ of the total charged current cross section [41] for the same $Q^{2}$ and $y$ ranges, with $x<0.05$.

The measured charged current cross section is compared with the prediction of the '2006 DPDF Fit A' to the neutral current diffractive DIS data described in section 5.4. The prediction is obtained by implementing the DPDFs extracted from the neutral current data in the RAPGAP [53] Monte Carlo generator. The light quark-initiated contributions are calculated at lowest order and the $\bar{c} \rightarrow \bar{s}$ contribution is calculated using the $\mathcal{O}\left(\alpha_{s}\right)$ matrix element. Leading log $\left(Q^{2}\right)$ parton showers are used to approximate higher order QCD radiation. The resulting cross section prediction is $500 \mathrm{fb}$, which is compatible with the measurement. The experimental uncertainties on the DPDFs and the theoretical uncertainties detailed in table 2 lead to negligible errors on the predictions by comparison with the statistical error on the measured cross section.

The charged current cross section measurement is shown differentially in $x_{\mathbb{P}}, \beta$ and $Q^{2}$ in figures $15 \mathrm{a}-\mathrm{c}$, respectively. The numerical values are given in table 6 . In all cases, the 
predictions derived from the DPDFs of section 5.4 are in agreement with the measurements. The charged current data are thus consistent with the singlet quark distribution assumed in the DPDF fit, where all light quark and antiquark densities are taken to be equal, although the large statistical uncertainties preclude strong conclusions. The contribution in the model from the sub-leading exchange is shown as a dashed line in the figures. It contributes at the $15 \%$ level for $x_{\mathbb{P}}>0.015$ and is negligible at lower $x_{\mathbb{P}}$.

\section{Comparison between Diffractive and Inclusive DIS}

In hadronic scattering, close connections have been drawn between the diffractive and the total cross sections, for example via the generalisation of the optical theorem to diffractive dissociation processes [85]. These connections are carried forward into many models of low $x$ DIS $[14,15,18]$. Comparing the $Q^{2}$ and $x$ dynamics of the diffractive with the inclusive cross section is therefore a powerful means of developing our understanding of high energy QCD, comparing the properties of the DPDFs with their inclusive counterparts and testing models.

The evolution of the diffractive reduced cross section with $Q^{2}$ is compared with that of the inclusive DIS reduced cross section $\sigma_{r}$ by forming the ratio

$$
\left.\frac{\sigma_{r}^{D(3)}\left(x_{\mathbb{P}}, x, Q^{2}\right)}{\sigma_{r}\left(x, Q^{2}\right)}\right|_{x, x_{\mathbb{P}}}
$$

at fixed $x$ and $x_{\mathbb{P}}$, using parameterisations of the $\sigma_{r}$ data from ${ }^{9}[41,47]$. This ratio, which was also studied in [10], is shown multiplied by $x_{\mathbb{P}}$ in figures 16 and 17, as a function of $Q^{2}$ for all measured $x_{I P}$ and $x=\beta x_{I P}$ values. In order to compare the $Q^{2}$ dependences of the diffractive and the inclusive cross sections quantitatively, the logarithmic derivative of their ratio, $b_{\mathrm{R}}\left(x, x_{\mathbb{P}}\right)=\frac{\partial}{\partial \ln Q^{2}}\left(\sigma_{r}^{D(3)} / \sigma_{r}\right)_{x, x_{\mathbb{P}}}$, is extracted through fits of a similar form to equation 10 , whereby

$$
\left.\frac{\sigma_{r}^{D(3)}\left(x_{\mathbb{P}}, x, Q^{2}\right)}{\sigma_{r}\left(x, Q^{2}\right)}\right|_{x, x_{\mathbb{P}}}=a_{\mathrm{R}}\left(x, x_{\mathbb{P}}\right)+b_{\mathrm{R}}\left(x, x_{\mathbb{P}}\right) \ln Q^{2}
$$

The fits are overlayed on the data in figures 16 and 17. The resulting values of $b_{\mathrm{R}}$ are shown in figure 18 , where they are divided by the flux factor $f_{\mathbb{P} / p}\left(x_{\mathbb{P}}\right)$ (equation 14 ), to allow comparisons between different $x_{\mathbb{P}}$ values. Since the dominant uncertainties arise from the diffractive data, the statistical fluctuations in figure 18 reproduce those of figure $7 \mathrm{a}$.

The ratio of the diffractive to the inclusive cross section is remarkably flat as a function of $Q^{2}$ for most $x$ and $x_{I P}$ values, such that the $\ln Q^{2}$ derivative of the ratio is consistent with zero. At the highest $\beta \gtrsim 0.3$, where $x$ approaches $x_{\mathbb{P}}$, the ratio falls with increasing $Q^{2}$ and the $\ln Q^{2}$ derivative becomes negative. This occurs in a manner which depends to good approximation on $\beta$ only; at fixed $\beta$, there is no significant dependence of the logarithmic derivative on $x_{\mathbb{P}}$.

\footnotetext{
${ }^{9}$ The inclusive reduced cross section is denoted $\tilde{\sigma}_{\mathrm{NC}}$ in [41].
} 
The compatibility of the $\ln Q^{2}$ derivative of the ratio with zero over much of the kinematic range implies that $1 / \sigma_{r}^{D(3)} \cdot \partial \sigma_{r}^{D(3)} / \partial \ln Q^{2} \simeq 1 / \sigma_{r} \cdot \partial \sigma_{r} / \partial \ln Q^{2}$. Whereas the diffractive and inclusive reduced cross sections are closely related to their respective quark densities, the $\ln Q^{2}$ derivatives are approximately proportional to the relevant gluon densities in regions where the $Q^{2}$ evolution is dominated by the $g \rightarrow q \bar{q}$ splitting (see section 4.1 and [86]). The compatibility of $b_{\mathrm{R}}$ with zero for $\beta \lesssim 0.3$ thus implies that the ratio of the quark to the gluon density is similar in the diffractive and inclusive cases when considered at the same $x$ values. Indeed, global fits to inclusive DIS data $[41,78,79]$ yield gluon fractions of approximately $70 \%$ at low $x$, compatible with the results of section 5.4. At higher $\beta \gtrsim 0.3$, where the DPDFs develop a more complicated structure (see figure 11) and the $q \rightarrow q g$ splitting becomes important in the evolution (see section 4.1 ), $b_{\mathrm{R}}$ becomes negative.

The ratio defined in equation 18 can also be plotted as a function of $x$ (or $\beta$ ) with $x_{\mathbb{P}}$ and $Q^{2}$ fixed. However, this results in a complicated dependence, which is driven by the high $\beta$ structure of the diffractive reduced cross section (figures 2-6a).

The $x$ dependence of the ratio of the diffractive to the inclusive cross section has been studied previously ${ }^{10}$ at fixed $M_{\mathrm{x}}[8,9,11]$ rather than fixed $x_{\mathbb{P}}$. Using the diffractive data as presented in figure 8 , the quantity

$$
(1-\beta) \cdot \frac{x_{\mathbb{P}} \cdot \sigma_{r}^{D(3)}\left(x_{\mathbb{P}}, \beta, Q^{2}\right)}{\sigma_{r}\left(x, Q^{2}\right)} \equiv M_{\mathrm{x}}^{2} \frac{\mathrm{d} \sigma\left(\gamma^{*} p \rightarrow X Y\right)}{\mathrm{d} M_{\mathrm{x}}^{2}} / \sigma_{\mathrm{tot}}\left(\gamma^{*} p \rightarrow X^{\prime}\right)
$$

is formed. Assuming proton vertex factorisation and neglecting contributions from sub-leading exchanges, the generalised optical theorem [85] predicts that this ratio is independent of $Q^{2}$ and depends only weakly on $\beta=Q^{2} /\left(Q^{2}+M_{\mathrm{x}}\right)$ and $x \simeq Q^{2} / W^{2}$ for sufficiently large $M_{\mathrm{x}}$. In models in which both the diffractive and the inclusive cross sections are governed by a universal pomeron [84], the remaining weak $x$ dependence of the ratio arises due to the deviations from unity of the pomeron trajectory.

The ratio defined in equation 20 is shown in figure 19 as a function of $x$ in bins of fixed $Q^{2}$, $\beta$ and hence $M_{\mathrm{x}}$. In order to simplify the interpretation, data points are excluded if, according to the QCD fit in section 5, the sub-leading exchange contribution is larger than $10 \%$ or $\sigma_{r}^{D(3)}$ differs by more than $10 \%$ from $F_{2}^{D(3)}$ due to the influence of $F_{L}^{D(3)}$. Only $Q^{2}$ and $\beta$ values for which there are at least two remaining data points are shown. The ratio in equation 20 is indeed approximately constant throughout the full kinematic range, except at large $\beta$ values (the low $M_{\mathrm{x}}$ "non-triple-Regge" region in which [85] is not applicable). In particular, the $x$ (and hence the $W$ ) dependence at fixed $Q^{2}, \beta$ and $M_{\mathrm{x}}$ is strikingly flat, substantiating the conclusions of $[8,9,11]$.

Expressed in terms of Regge trajectories, the ratio of cross sections shown in figure 19 is proportional to $x^{-\kappa}$, where $\kappa=2\left\langle\alpha_{\mathbb{P}}(t)\right\rangle-\alpha_{\mathbb{P}}^{\text {incl }}(0)-1$. Here $\alpha_{\mathbb{P}}(t)$ is the effective pomeron trajectory for diffractive DIS and $\alpha_{\mathbb{P}}^{\text {incl }}(0)$ is the effective pomeron intercept governing inclusive scattering. Analysis of inclusive DIS data has shown that $\alpha_{\mathbb{P}}^{\text {incl }}(0)$ is not universal, but varies with $Q^{2}$ according to $\alpha_{\mathbb{P}}^{\text {incl }}(0) \simeq 1+0.048 \ln \left(Q^{2} /[0.292 \mathrm{GeV}]^{2}\right)$ [87]. A prediction is overlaid

\footnotetext{
${ }^{10}$ The analyses in $[8,9,11]$ differ from that described here in that the data were plotted as a function of $W \simeq$ $\sqrt{Q^{2} / x}$. In $[8,11]$, the results were also shown integrated over ranges in $M_{\mathrm{x}}$, whereas they are presented here at fixed $M_{\mathrm{x}}$ values.
} 
in figure 19, where it is assumed that the diffractive $\alpha_{\mathbb{P}}(0)$ depends on $Q^{2}$ in the same way as $\alpha_{\mathbb{P}}^{\text {incl }}(0)$ and that $\alpha_{\mathbb{P}}^{\prime}=0.06 \mathrm{GeV}^{-2}$ (see section 5.2). Similar results are obtained with $\alpha_{\mathbb{P}}^{\prime}=0$ or $\alpha_{\mathbb{P}}^{\prime}=0.25$. The normalisation of the prediction is obtained from separate fits to the data for each pair of $\beta$ and $Q^{2}$ values. The description of the data is poor ( $\chi^{2}=876$ based on statistical and uncorrelated systematic errors for 223 degrees of freedom). The ratio shown in figure 19 is also compared with a prediction where the inclusive data are described by the same $Q^{2}$ dependent $\alpha_{\mathbb{P}}^{\text {incl }}(0)$ of [87] and the diffractive $x$ dependence is determined by the flux factor defined in equation 14, such that $\alpha_{\mathrm{IP}}(0)=1.118$ (equation 16) independently of $Q^{2}$. A much improved description is obtained $\left(\chi^{2}=254\right)$, with an $x$ dependence of the ratio which changes slowly with $Q^{2}$, being approximately flat in the region of $Q^{2} \sim 15 \mathrm{GeV}^{2}$.

The ratio of cross sections shown in figure 19 is incompatible with a simple Regge approach to $\gamma^{*} p$ scattering, where both the diffractive and the inclusive cross sections are driven by the exchange of the same pomeron trajectory, even if that trajectory is allowed to change with $Q^{2}$. There is thus no simple relationship within a Regge model between the diffractive and inclusive cross sections. The flatness of the ratio of cross sections is natural if rapidity gap formation is a random process $[14,15]$ and has also been interpreted in colour dipole models [18].

\section{Summary}

The reduced semi-inclusive diffractive cross section $\sigma_{r}^{D(3)}\left(x_{\mathbb{P}}, \beta, Q^{2}\right)$ is measured for the process $e p \rightarrow e X Y$ under the conditions $M_{\mathrm{Y}}<1.6 \mathrm{GeV}$ and $|t|<1 \mathrm{GeV}^{2}$ for various fixed values of $x_{\mathbb{P}}$ in the range $0.0003<x_{\mathbb{P}}<0.03$. The data span nearly three orders of magnitude in $Q^{2}$ from $3.5 \mathrm{GeV}^{2}$ to $1600 \mathrm{GeV}^{2}$ and cover the range $0.0017 \leq \beta \leq 0.8$. In the best measured region, the statistical and point-to-point systematic uncertainties are at the level of $5 \%$ each, with an additional normalisation uncertainty of $6 \%$. The kinematic dependences of the ratio of the diffractive to the inclusive reduced cross section are also studied.

Up to small deviations at large $x_{\mathbb{P}}$ and low $\beta$, which are consistent with expectations from the presence of a sub-leading exchange, the $\beta$ and $Q^{2}$ dependences of the diffractive data change only in normalisation at different $x_{\mathbb{P}}$ values. This remarkable feature is compatible with a factorisable proton vertex. The variation of $\sigma_{r}^{D(3)}$ with $x_{\mathbb{P}}$ can be expressed in terms of an effective pomeron trajectory with intercept $\alpha_{\mathbb{P}}(0)=1.118 \pm 0.008$ (exp.) ${ }_{-0.010}^{+0.029}$ (model) if $\alpha_{\mathbb{P}}^{\prime}=0.06{ }_{-0.06}^{+0.19} \mathrm{GeV}^{-2}$ is taken from H1 Forward Proton Spectrometer data. The $x$ (or $x_{\mathbb{P}}$ ) dependence of the diffractive cross section at fixed $\beta$ and $Q^{2}$ is similar to that of the inclusive cross section. The diffractive and inclusive $x$ dependences cannot be interpreted with a single $Q^{2}$ dependent effective pomeron trajectory.

The $\beta$ and $Q^{2}$ dependences of $\sigma_{r}^{D(3)}$ are interpreted in terms of diffractive parton distribution functions (DPDFs), obtained through an NLO DGLAP QCD fit. The DPDFs correspond to integrals over the measured $M_{\mathrm{Y}}$ and $t$ ranges and are valid in the region $Q^{2} \geq 8.5 \mathrm{GeV}^{2}$, $\beta \leq 0.8$ and $M_{\mathrm{x}}>2 \mathrm{GeV}$. At low $\beta \lesssim 0.3$, the diffractive data exhibit a rather fast rise with increasing $Q^{2}$ at fixed $x_{\mathbb{P}}$ and $x$. This rise of the diffractive cross section is very similar to that of the inclusive cross section at the same $x$ values, implying that the ratio of quarks to gluons is similar in the diffractive and inclusive cases. The low $\beta$ data give good constraints on 
the diffractive quark singlet and gluon densities at low momentum fractions $z$, with combined experimental and theoretical uncertainties of typically $5-10 \%$ and $15 \%$, respectively. The gluon density dominates the DPDFs in this region. At larger $z$, the diffractive quark density remains well constrained by the fit, whereas the sensitivity to the gluon density becomes increasingly poor.

At high $Q^{2}$, charged current scattering is used to test the assumptions on the quark flavour decomposition of the DPDFs. Total and single differential diffractive $e^{+} p$ charged current cross sections are measured and are well described by predictions based on the DPDFs extracted from the neutral current data, though the current level of experimental precision $(35 \%$ for the total cross section) is low. The DPDFs will provide important input to future tests of the factorisation properties of diffraction and to the prediction of cross sections for diffractive processes at HERA, the LHC and elsewhere.

\section{Acknowledgements}

We are grateful to the HERA machine group whose outstanding efforts have made this experiment possible. We thank the engineers and technicians for their work in constructing and now maintaining the $\mathrm{H} 1$ detector, our funding agencies for financial support, the DESY technical staff for continual assistance and the DESY directorate for support and for the hospitality which they extend to the non DESY members of the collaboration. 


\section{References}

[1] A. Brandt et al. [UA8 Collaboration], Phys. Lett. B 297 (1992) 417.

[2] M. Derrick et al. [ZEUS Collaboration], Phys. Lett. B 315 (1993) 481;

T. Ahmed et al. [H1 Collaboration], Nucl. Phys. B 429 (1994) 477.

[3] T. Ahmed et al. [H1 Collaboration], Nucl. Phys. B 439 (1995) 471 [hep-ex/9503001].

[4] T. Ahmed et al. [H1 Collaboration], Phys. Lett. B 348 (1995) 681 [hep-ex/9503005].

[5] C. Adloff et al. [H1 Collaboration], Z. Phys. C 76 (1997) 613 [hep-ex/9708016].

[6] H1 Collaboration, "Diffractive Deep-Inelastic Scattering with a Leading Proton at HERA”, DESY 06-048, submitted to Eur. Phys. J. C.

[7] M. Derrick et al. [ZEUS Collaboration], Z. Phys. C 68 (1995) 569 [hep-ex/9505010].

[8] J. Breitweg et al. [ZEUS Collaboration], Eur. Phys. J. C 6 (1999) 43 [hep-ex/9807010].

[9] S. Chekanov et al. [ZEUS Collaboration], Eur. Phys. J. C 25 (2002) 169 [hep-ex/0203039].

[10] S. Chekanov et al. [ZEUS Collaboration], Eur. Phys. J. C 38 (2004) 43 [hep-ex/0408009].

[11] S. Chekanov et al. [ZEUS Collaboration], Nucl. Phys. B 713 (2005) 3 [hep-ex/0501060].

[12] A. Hebecker, Phys. Rept. 331 (2000) 1 [hep-ph/9905226].

[13] N. Nikolaev and B. Zakharov, Z. Phys. C 53 (1992) 331.

[14] W. Buchmüller and A. Hebecker, Phys. Lett. B 355 (1995) 573 [hep-ph/9504374];

W. Buchmüller, T. Gehrmann and A. Hebecker, Nucl. Phys. B 537 (1999) 477 [hep$\mathrm{ph} / 9808454]$.

[15] A. Edin, G. Ingelman and J. Rathsman, Phys. Lett. B 366 (1996) 371 [hep-ph/9508386];

A. Edin, G. Ingelman and J. Rathsman, Z. Phys. C 75 (1997) 57 [hep-ph/9605281];

J. Rathsman, Phys. Lett. B 452 (1999) 364 [hep-ph/9812423];

S. Brodsky, R. Enberg, P. Hoyer and G. Ingelman, Phys. Rev. D 71 (2005) 074020 [hep$\mathrm{ph} / 0409119]$.

[16] F. Hautmann, Z. Kunszt and D. Soper, Nucl. Phys. B 563 (1999) 153 [hep-ph/9906284];

F. Hautmann and D. Soper, Phys. Rev. D 63 (2000) 011501 [hep-ph/0008224].

[17] J. Bartels, J. Ellis, H. Kowalski and M. Wüsthoff, Eur. Phys. J. C 7 (1999) 443 [hep$\mathrm{ph} / 9803497]$.

[18] K. Golec-Biernat and M. Wüsthoff, Phys. Rev. D 59 (1999) 014017 [hep-ph/9807513];

K. Golec-Biernat and M. Wüsthoff, Phys. Rev. D 60 (1999) 114023 [hep-ph/9903358];

J. Bartels, K. Golec-Biernat and H. Kowalski, Phys. Rev. D 66 (2002) 014001 [hep$\mathrm{ph} / 0203258]$. 
[19] A. Martin, M. Ryskin and G. Watt, Phys. Rev. D 70 (2004) 091502 [hep-ph/0406225]; G. Watt, A. Martin and M. Ryskin, Phys. Lett. B 627 (2005) 97 [hep-ph/0508093].

[20] J. Collins, Phys. Rev. D 57 (1998) 3051 [Erratum-ibid. D 61 (2000) 019902] [hep$\mathrm{ph} / 9709499]$.

[21] A. Berera and D. Soper, Phys. Rev. D 50 (1994) 4328 [hep-ph/9403276].

[22] A. Berera and D. Soper, Phys. Rev. D 53 (1996) 6162 [hep-ph/9509239].

[23] Z. Kunszt and W. J. Stirling, Proc. of the Workshop on Deep-Inelastic Scattering and Related Phenomena DIS96, eds. G. D’Agostini, A. Nigro, Rome (1996) 240 [hep$\mathrm{ph} / 9609245]$.

[24] L. Trentadue and G. Veneziano, Phys. Lett. B 323 (1994) 201;

M. Grazzini, L. Trentadue and G. Veneziano, Nucl. Phys. B 519 (1998) 394 [hep$\mathrm{ph} / 9709452]$.

[25] E. Feinberg and I. Pomeranchuk, Suppl. Nuovo. Cimento. 3 (1956) 652;

V. Gribov, JETP Lett. 41 (1961) 667.

[26] G. Ingelman and P. Schlein, Phys. Lett. B 152 (1985) 256;

A. Donnachie and P. Landshoff, Phys. Lett. B 191 (1987) 309 [Erratum-ibid. B 198 (1987) 590].

[27] J. Breitweg et al. [ZEUS Collaboration], Eur. Phys. J. C 5 (1998) 41 [hep-ex/9804013].

[28] K. Golec-Biernat and J. Kwiecinski, Phys. Lett. B 353 (1995) 329 [hep-ph/9504230].

[29] T. Gehrmann and W. J. Stirling, Z. Phys. C 70 (1996) 89 [hep-ph/9503351].

[30] L. Alvero, J. Collins, J. Terron and J. Whitmore, Phys. Rev. D 59 (1999) 074022 [hep$\mathrm{ph} / 9805268]$.

[31] C. Royon et al., Phys. Rev. D 63 (2001) 074004 [hep-ph/0010015].

[32] A. Martin, M. Ryskin and G. Watt, Eur. Phys. J. C 37 (2004) 285 [hep-ph/0406224];

A. Martin, M. Ryskin and G. Watt, Eur. Phys. J. C 44 (2005) 69 [hep-ph/0504132];

G. Watt, Proc. of the Workshop on New Trends in HERA Physics, ed. G. Grindhammer et al., Ringberg Castle, Germany (2005) 303 [hep-ph/0511333].

[33] C. Adloff et al. [H1 Collaboration], Eur. Phys. J. C 6 (1999) 421 [hep-ex/9808013];

C. Adloff et al. [H1 Collaboration], Eur. Phys. J. C 20 (2001) 29 [hep-ex/0012051].

[34] C. Adloff et al. [H1 Collaboration], Phys. Lett. B 520 (2001) 191 [hep-ex/0108047].

[35] J. Collins, L. Frankfurt and M. Strikman, Phys. Lett. B 307 (1993) 161 [hep-ph/9212212]. 
[36] F. Abe et al. [CDF Collaboration], Phys. Rev. Lett. 79 (1997) 2636;

F. Abe et al. [CDF Collaboration], Phys. Rev. Lett. 78 (1997) 2698 [hep-ex/9703010];

B. Abbott et al. [D0 Collaboration], Phys. Lett. B 531 (2002) 52 [hep-ex/9912061];

T. Affolder et al. [CDF Collaboration], Phys. Rev. Lett. 84 (2000) 5043;

T. Affolder et al. [CDF Collaboration], Phys. Rev. Lett. 85 (2000) 4215;

V. Abazov et al. [D0 Collaboration], Phys. Lett. B 574 (2003) 169 [hep-ex/0308032].

[37] J. Bjorken, Phys. Rev. D 47 (1993) 101;

E. Gotsman, E. Levin and U. Maor, Phys. Lett. B 309 (1993) 199 [hep-ph/9302248];

E. Gotsman, E. Levin and U. Maor, Phys. Lett. B 438 (1998) 229 [hep-ph/9804404];

B. Cox, J. Forshaw and L. Lönnblad, JHEP 9910 (1999) 023 [hep-ph/9908464];

A. Kaidalov, V. Khoze, A. Martin and M. Ryskin, Phys. Lett. B 567 (2003) 61 [hep$\mathrm{ph} / 0306134]$.

[38] M. Arneodo et al., Proc. of the HERA-LHC workshop, eds. A. De Roeck, H. Jung, CERN2005-014 (2005) 417 [hep-ex/0601013].

[39] V. Gribov and L. Lipatov, Sov. J. Nucl. Phys. 15 (1972) 438 [Yad. Fiz. 15 (1972) 781];

V. Gribov and L. Lipatov, Sov. J. Nucl. Phys. 15 (1972) 675 [Yad. Fiz. 15 (1972) 1218];

Y. Dokshitzer, Sov. Phys. JETP 46 (1977) 641 [Zh. Eksp. Teor. Fiz. 73 (1977) 1216];

G. Altarelli and G. Parisi, Nucl. Phys. B 126 (1977) 298.

[40] W. Furmanski and R. Petronzio, Z. Phys. C 11 (1982) 293.

[41] C. Adloff et al. [H1 Collaboration], Eur. Phys. J. C 30 (2003) 1 [hep-ex/0304003].

[42] B. Andrieu et al. [H1 Calorimeter Group], Nucl. Instrum. Meth. A 336 (1993) 499;

B. Andrieu et al. [H1 Calorimeter Group], Nucl. Instrum. Meth. A 350 (1994) 57.

[43] I. Abt et al. [H1 Collaboration], Nucl. Instrum. Meth. A 386 (1997) 310;

I. Abt et al. [H1 Collaboration], Nucl. Instrum. Meth. A 386 (1997) 348.

[44] R. Appuhn et al. [H1 SPACAL Group], Nucl. Instrum. Meth. A 386 (1997) 397.

[45] C. Johnson, Ph.D. Thesis, University of Birmingham (2002), available from http://www-h1.desy.de/publications/theses_list.html;

Y. Coppens, Ph.D. Thesis, University of Birmingham (2004), available from http://www-h1.desy.de/publications/theses_list.html.

[46] P. Laycock, Ph.D. Thesis, University of Liverpool (2003), available from http://www-h1.desy.de/publications/theses_list.html.

[47] C. Adloff et al. [H1 Collaboration], Eur. Phys. J. C 21 (2001) 33 [hep-ex/0012053].

[48] C. Adloff et al. [H1 Collaboration], Z. Phys. C 74 (1997) 221 [hep-ex/9702003].

[49] A. Blondel and F. Jacquet, Proc. of the Study of an ep Facility for Europe, ed. U. Amaldi, DESY 79/48 (1979) 391.

[50] S. Bentvelsen et al., Proceedings of the Workshop "Physics at HERA", eds. W. Buchmüller, G. Ingelman, DESY (1992) 23; C. Hoeger, ibid. 43. 
[51] B. List, B. List and A. Mastroberardino, Proc. of the Workshop on Monte Carlo Generators for HERA Physics, eds. A. Doyle, G. Grindhammer, G. Ingelman, H. Jung, DESY-PROC1999-02 (1999) 396.

[52] S. Eidelman et al. [Particle Data Group], Phys. Lett. B 592 (2004) 1.

[53] H. Jung, Comput. Phys. Commun. 86 (1995) 147; (see also http://www.desy.de/ jung/rapgap.html).

[54] M. Bengtsson and T. Sjöstrand, Z. Phys. C 37 (1988) 465.

[55] B. Andersson, G. Gustafson, G. Ingelman and T. Sjöstrand, Phys. Rept. 97 (1983) 31.

[56] T. Sjöstrand, Comput. Phys. Commun. 82 (1994) 74.

[57] A. Kwiatkowski, H. Spiesberger and H. Möhring, Comput. Phys. Commun. 69 (1992) 155.

[58] G. Schüler and H. Spiesberger, Proc. of the Workshop on Physics at HERA, eds. W. Buchmüller, G. Ingelman, Hamburg, DESY (1992) 1419.

[59] C. Adloff et al. [H1 Collaboration], Eur. Phys. J. C 13 (2000) 609 [hep-ex/9908059].

[60] R. Engel and J. Ranft, Phys. Rev. D 54 (1996) 4244 [hep-ph/9509373].

[61] A. Courau and P. Kessler, Phys. Rev. D 46 (1992) 117.

[62] S. Baranov, O. Dünger, H. Shooshtari and J. Vermaseren, Proc. of the Workshop on Physics at HERA, eds. W. Buchmüller, G. Ingelman, Hamburg, DESY (1992) 1478.

[63] U. Baur, J. Vermaseren and D. Zeppenfeld, Nucl. Phys. B 375 (1992) 3.

[64] G. Gustafson, Phys. Lett. B 175 (1986) 453.

[65] L. Lönnblad, Comput. Phys. Commun. 71 (1992) 15.

[66] Data tables and detailed fit results can be found at http://www-h1.desy.de/h1/www/publications/htmlsplit/DESY-06-049.long.html.

[67] R. Nisius, Phys. Rept. 332 (2000) 165 [hep-ex/9912049].

[68] M. Gay Ducati, V. Goncalves and M. Machado, Phys. Lett. B 506 (2001) 52 [hep$\mathrm{ph} / 0010059]$

M. Gay Ducati, V. Goncalves and M. Machado, Nucl. Phys. A 697 (2002) 767 [hep$\mathrm{ph} / 0103245]$

M. Gay Ducati, E. Ferreiro, M. Machado and C. Salgado, Eur. Phys. J. C 24 (2002) 109 [hep-ph/0110184].

[69] M. Wüsthoff, Phys. Rev. D 56 (1997) 4311 [hep-ph/9702201].

[70] F. Low, Phys. Rev. D 12 (1975) 163;

S. Nussinov, Phys. Rev. Lett. 34 (1975) 1286. 
[71] A. Hebecker and T. Teubner, Phys. Lett. B 498 (2001) 16 [hep-ph/0010273].

[72] P. Newman and F.-P. Schilling, Proc. of the HERA-LHC workshop, eds. A. De Roeck, H. Jung, CERN-2005-014 (2005) 482 [hep-ex/0511032].

[73] M. Ryskin, Sov. J. Nucl. Phys. 52 (1990) 529 [Yad. Fiz. 52 (1990) 828, Nucl. Phys. Proc. Suppl. 18C (1990) 162];

E. Levin and M. Wüsthoff, Phys. Rev. D 50 (1994) 4306.

[74] W. Bardeen, A. Buras, D. Duke and T. Muta, Phys. Rev. D 18 (1978) 3998.

[75] E. Laenen, S. Riemersma, J. Smith and W. van Neerven, Nucl. Phys. B 392 (1993) 162;

E. Laenen, S. Riemersma, J. Smith and W. van Neerven, Nucl. Phys. B 392 (1993) 229.

[76] A. Martin, W. Stirling and R. Thorne, Phys. Lett. B 636 (2006) 259 [hep-ph/0603143].

[77] A. Buras and K. Gaemers, Nucl. Phys. B 132 (1978) 249.

[78] A. Martin, R. Roberts, W. J. Stirling and R. Thorne, Eur. Phys. J. C 23 (2002) 73 [hep$\mathrm{ph} / 0110215]$.

[79] J. Pumplin et al., JHEP 0207 (2002) 012 [hep-ph/0201195].

[80] J. Owens, Phys. Rev. D 30 (1984) 943.

[81] M. Glück, E. Reya and A. Vogt, Z. Phys. C 53 (1992) 651.

[82] C. Pascaud and F. Zomer, LAL-95-05.

[83] P. Newman, Proc. of International Europhysics Conference on High Energy Physics, Budapest, Hungary, July 2001, ed Horváth, Lévai, Patkós, JHEP PRHEP-hep2001/048, [hepex/0112034].

[84] A. Donnachie and P. Landshoff, Phys. Lett. B 296 (1992) 227 [hep-ph/9209205];

J. Cudell, K. Kang and S. Kim, Phys. Lett. B 395 (1997) 311 [hep-ph/9601336].

[85] A. Mueller, Phys. Rev. D 2 (1970) 2963.

[86] P. Johnson and W. K. Tung, Phys. Rev. D 16 (1977) 2769;

K. Prytz, Phys. Lett. B 311 (1993) 286.

[87] C. Adloff et al. [H1 Collaboration], Phys. Lett. B 520 (2001) 183 [hep-ex/0108035]. 
(a)

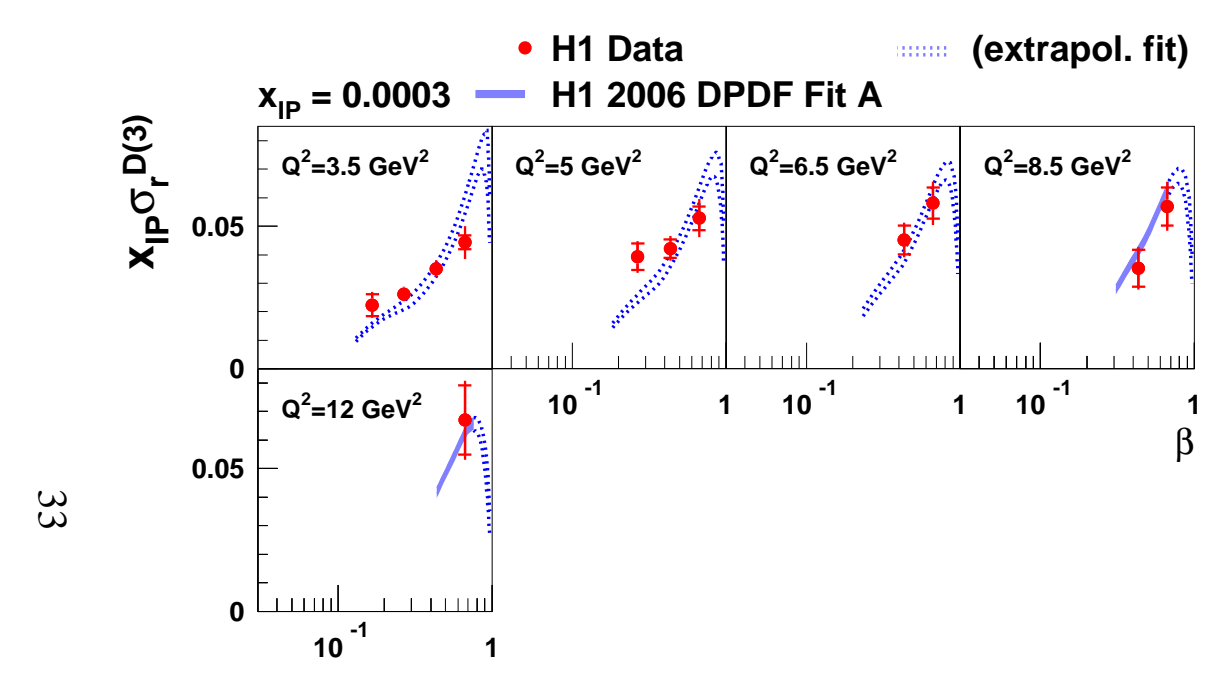

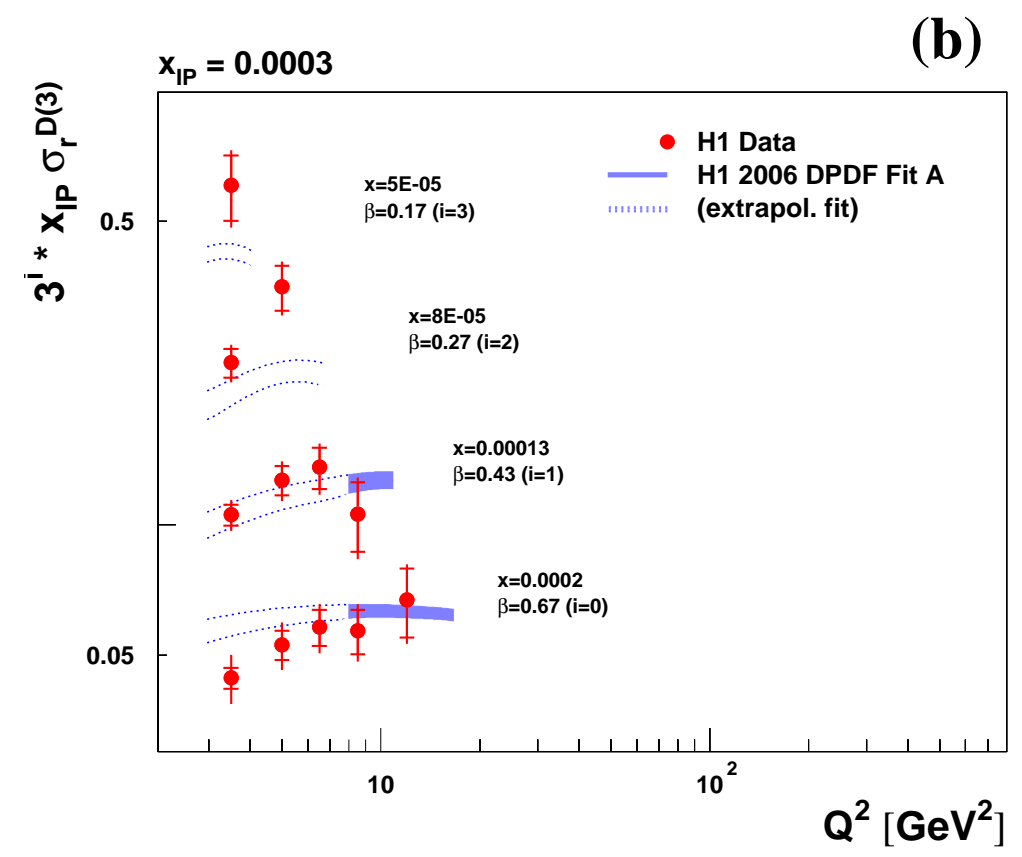

Figure 2: The $\beta$ and $Q^{2}$ dependences of the diffractive reduced cross section, multiplied by $x_{\mathbb{P}}$, at $x_{\mathbb{P}}=0.0003$. In (b) the data are multiplied by a further factor of $3^{i}$ for visibility, with $i$ as indicated. The inner and outer error bars on the data points represent the statistical and total uncertainties, respectively. Normalisation uncertainties are not shown. The data are compared with the reduced cross section at $E_{p}=820 \mathrm{GeV}$ derived from the results of 'H1 2006 DPDF Fit A', which is shown as a shaded error band (experimental uncertainties only) in kinematic regions which are included in the fit and as a pair of dashed lines in regions which are excluded from the fit. 
(a)
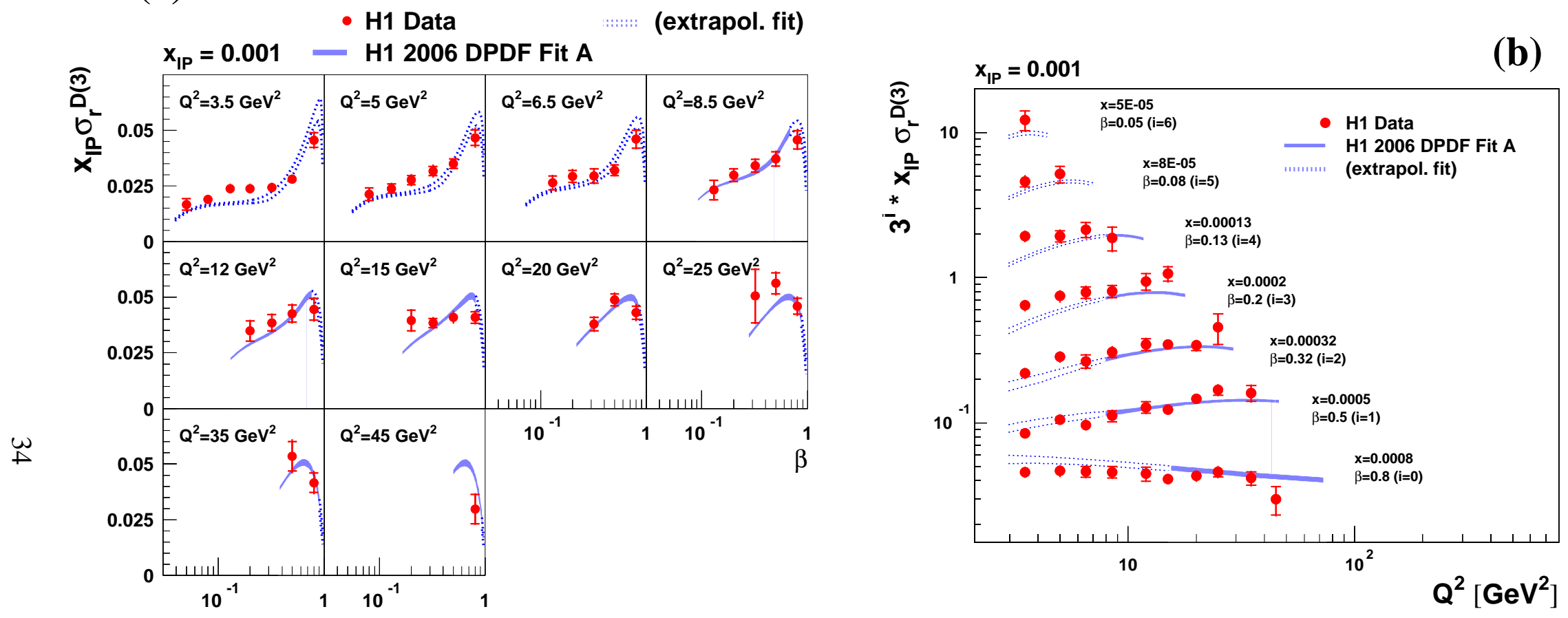

Figure 3: The $\beta$ and $Q^{2}$ dependences of the diffractive reduced cross section, multiplied by $x_{\mathbb{P}}$, at $x_{\mathbb{P}}=0.001$. See the caption of figure 2 for further details. 
(a)

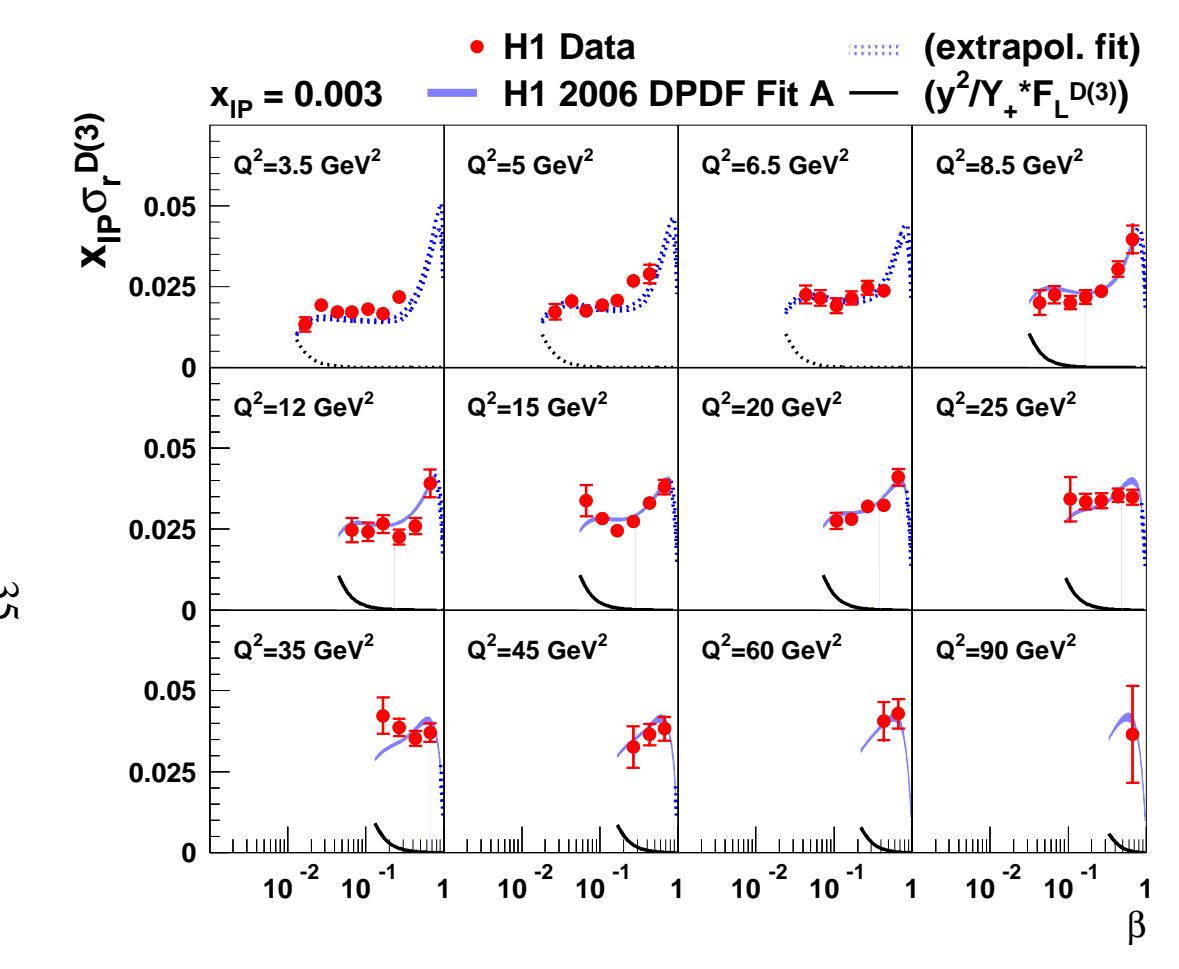

(b)

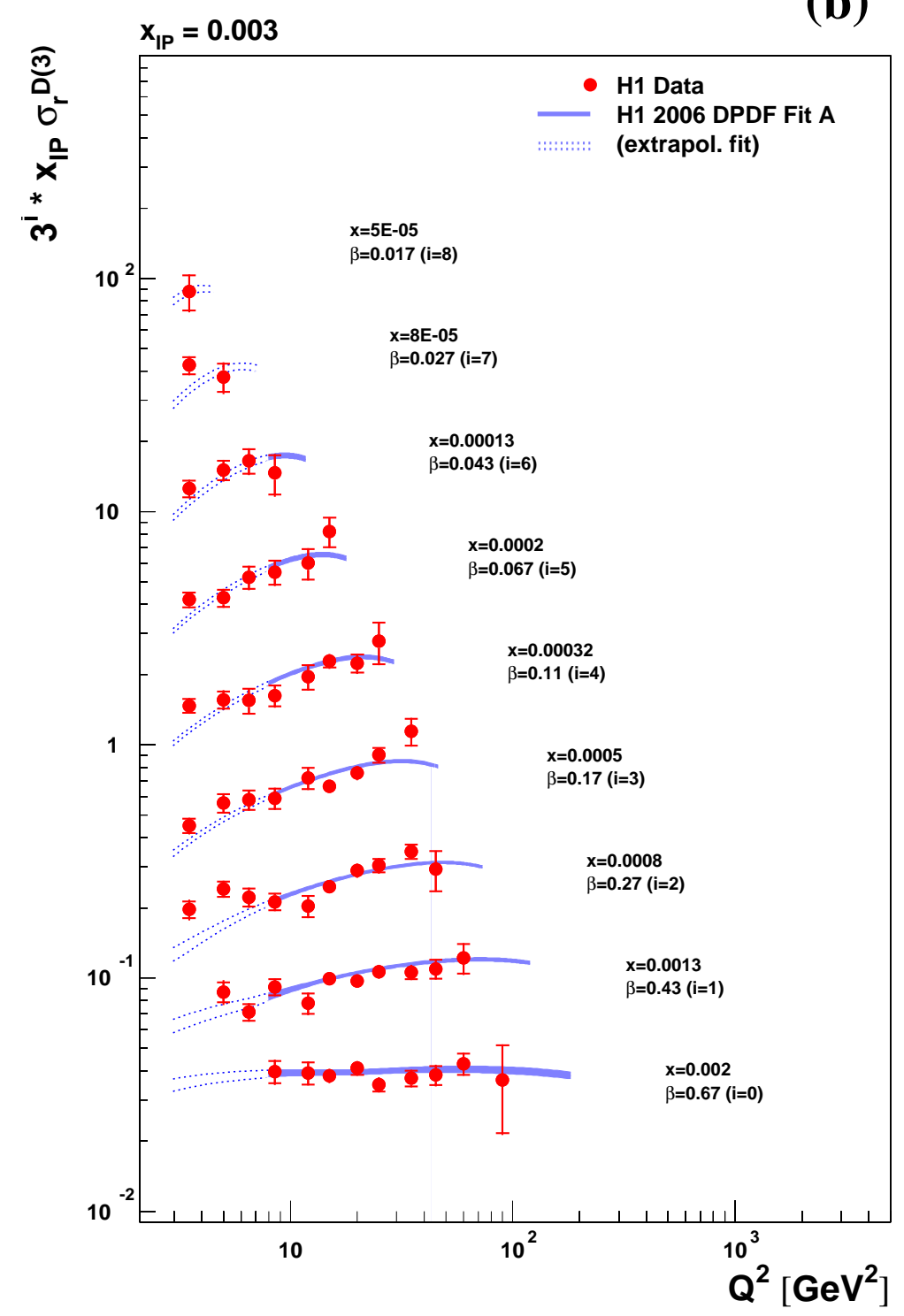

Figure 4: The $\beta$ and $Q^{2}$ dependences of the diffractive reduced cross section, multiplied by $x_{\mathbb{P}}$, at $x_{\mathbb{P}}=0.003$. In (a), the quantity $y^{2} / Y_{+} \cdot F_{L}^{D(3)}$ is also shown, as extracted from the 'H1 2006 DPDF Fit A'. Adding this quantity to the reduced cross section yields $F_{2}^{D(3)}$. See the caption of figure 2 for further details. 
(a)

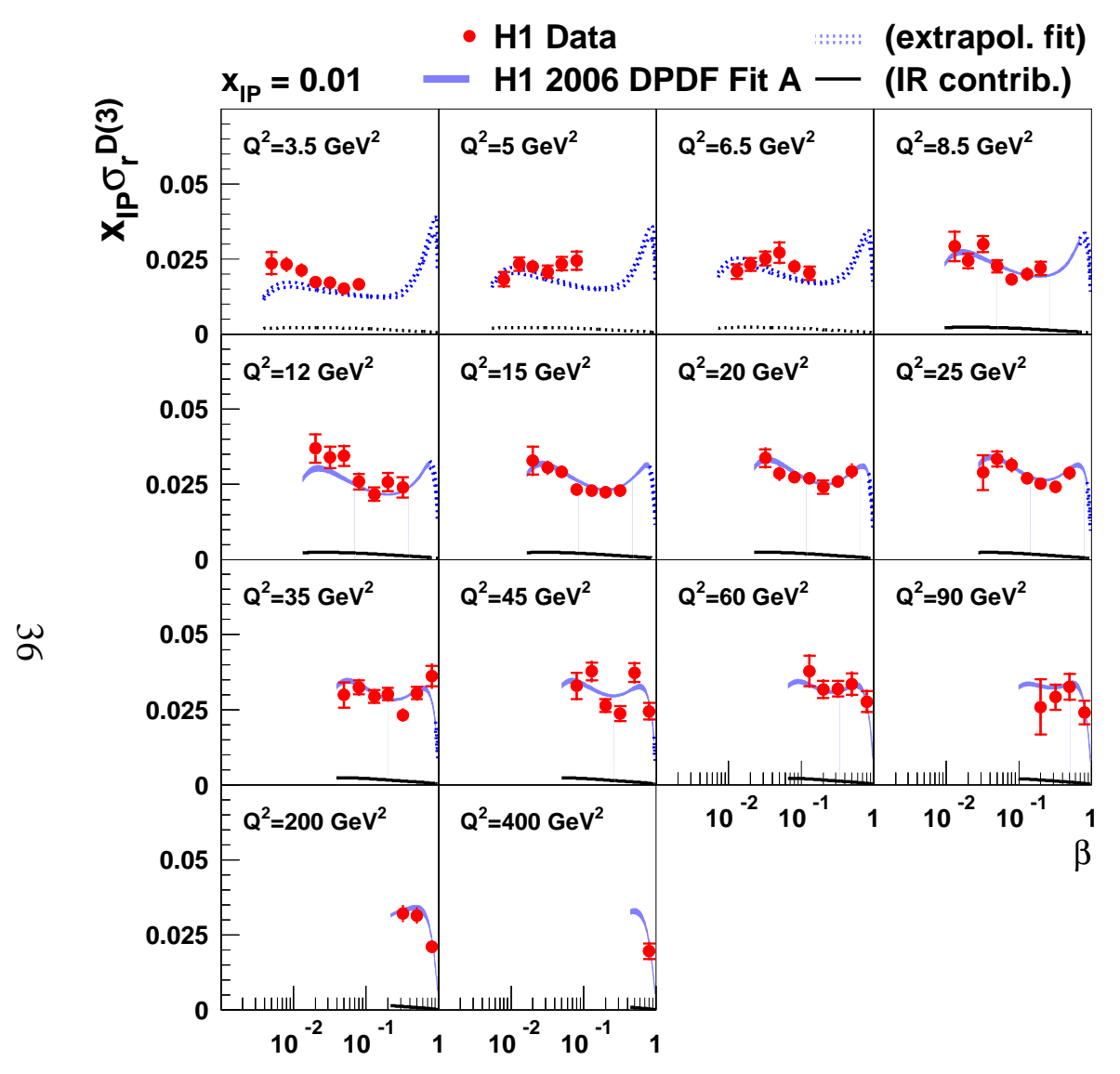

(b)

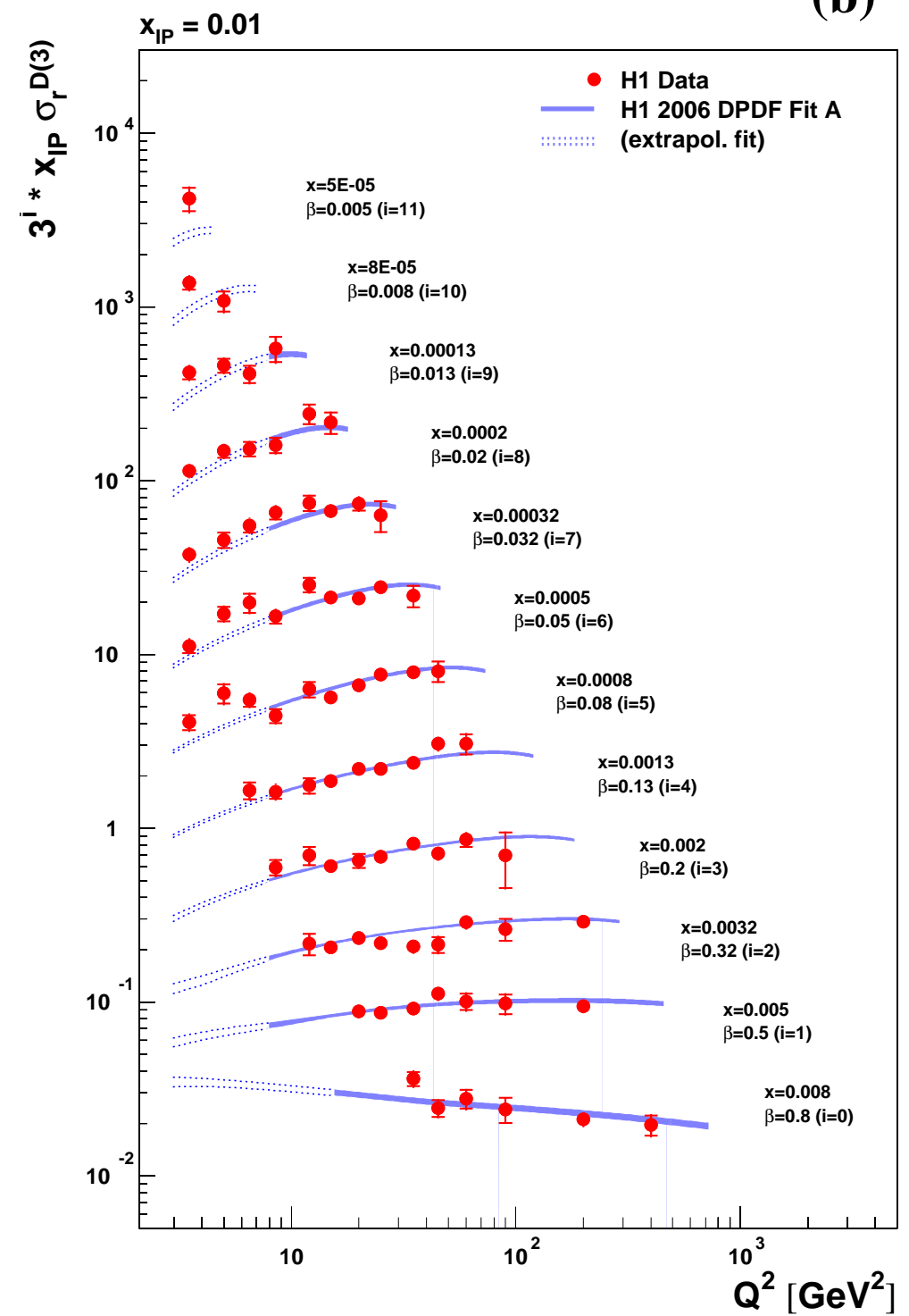

Figure 5: The $\beta$ and $Q^{2}$ dependences of the diffractive reduced cross section, multiplied by $x_{\mathbb{P}}$, at $x_{\mathbb{P}}=0.01$. In (a), the contribution of the sub-leading exchange alone according to the 'H1 2006 DPDF Fit A' is also shown. The data with $Q^{2} \leq 90 \mathrm{GeV}^{2}\left(Q^{2} \geq 200 \mathrm{GeV}^{2}\right)$ were obtained with $E_{p}=820 \mathrm{GeV}\left(E_{p}=920 \mathrm{GeV}\right)$. The fit results are shown for $E_{p}=820 \mathrm{GeV}$. See the caption of figure 2 for further details. 
(a)

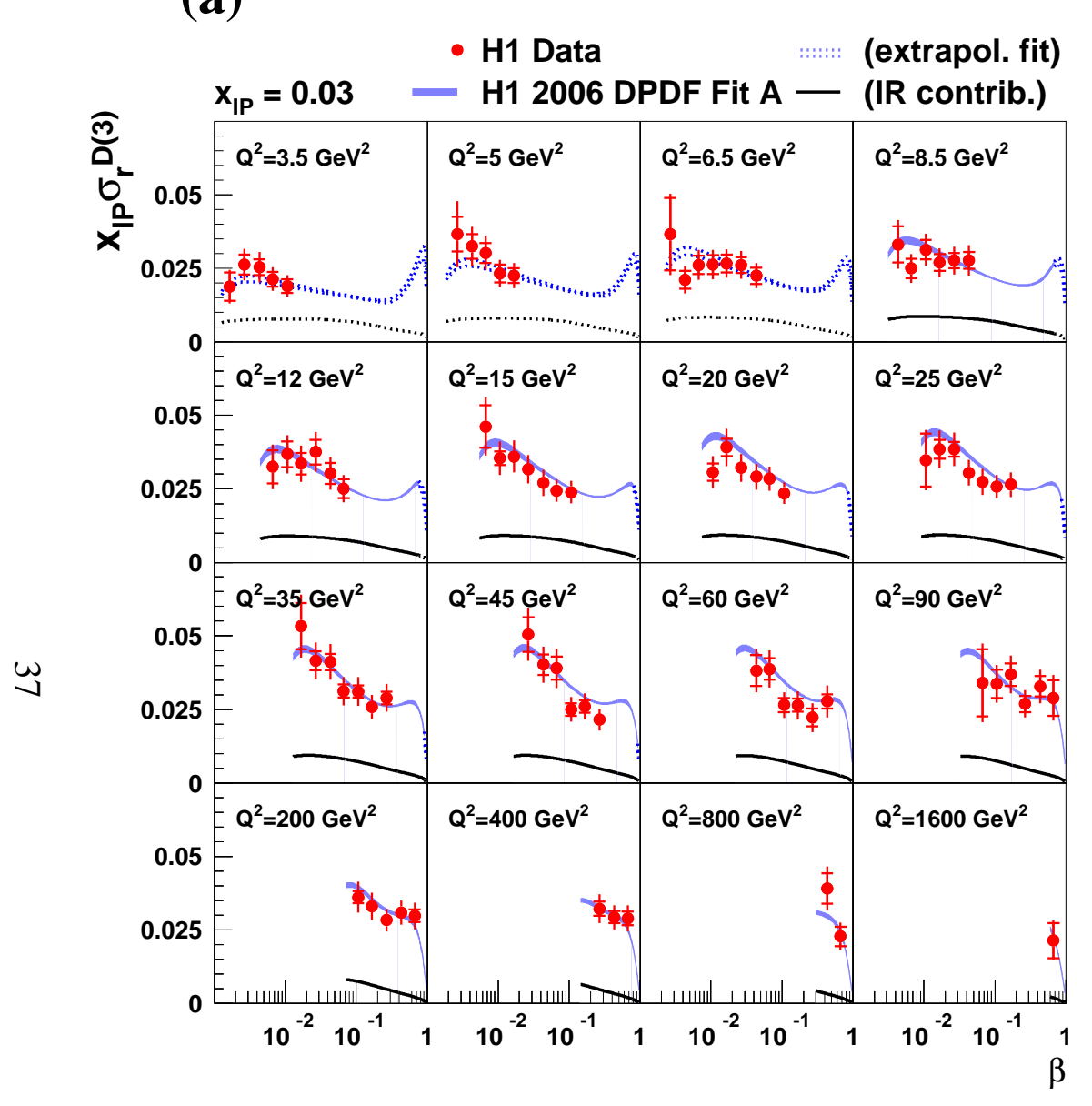

(b)

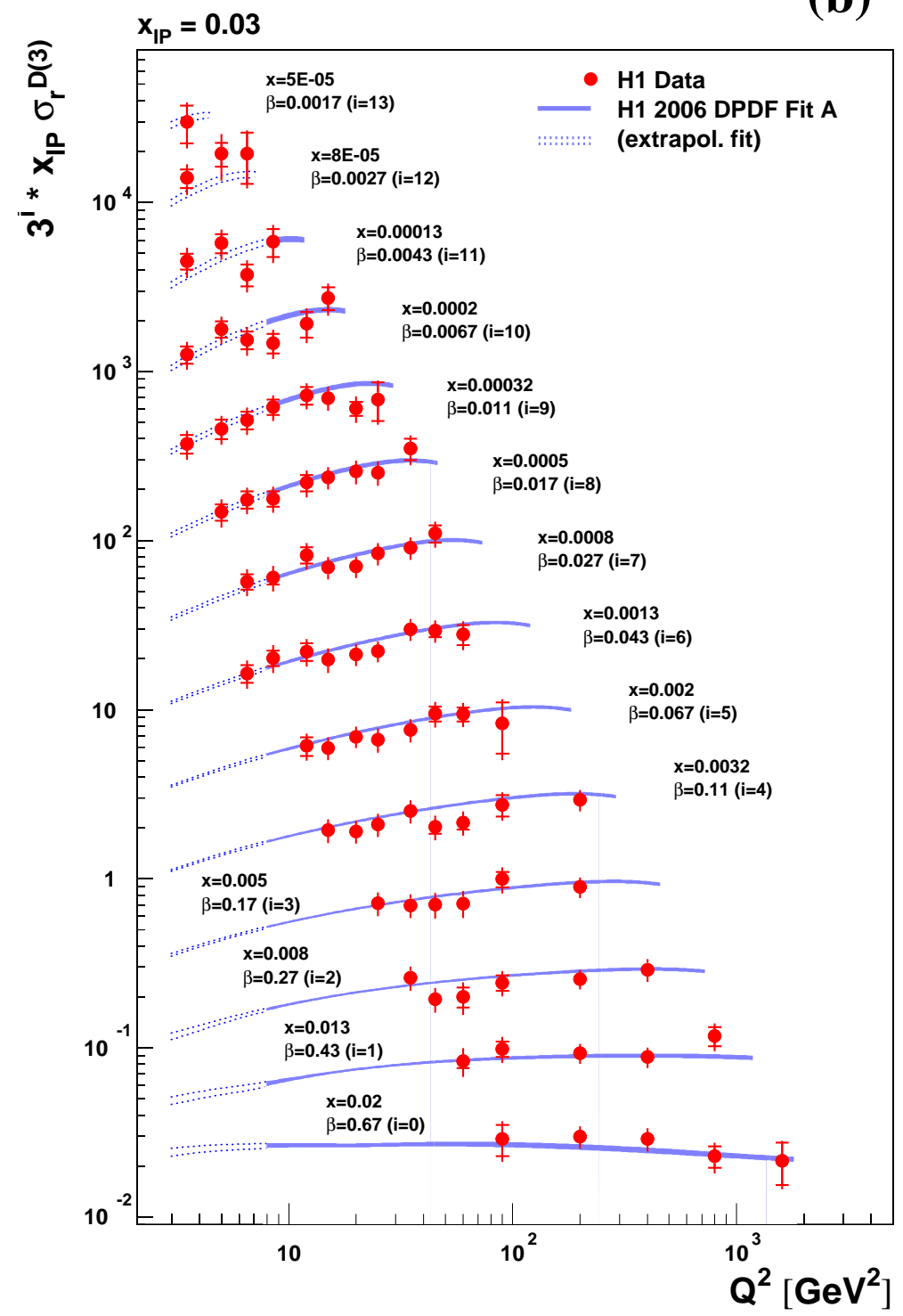

Figure 6: The $\beta$ and $Q^{2}$ dependences of the diffractive reduced cross section, multiplied by $x_{\mathbb{P}}$, at $x_{\mathbb{P}}=0.03$. See the captions of figures 2 and 5 for further details. 


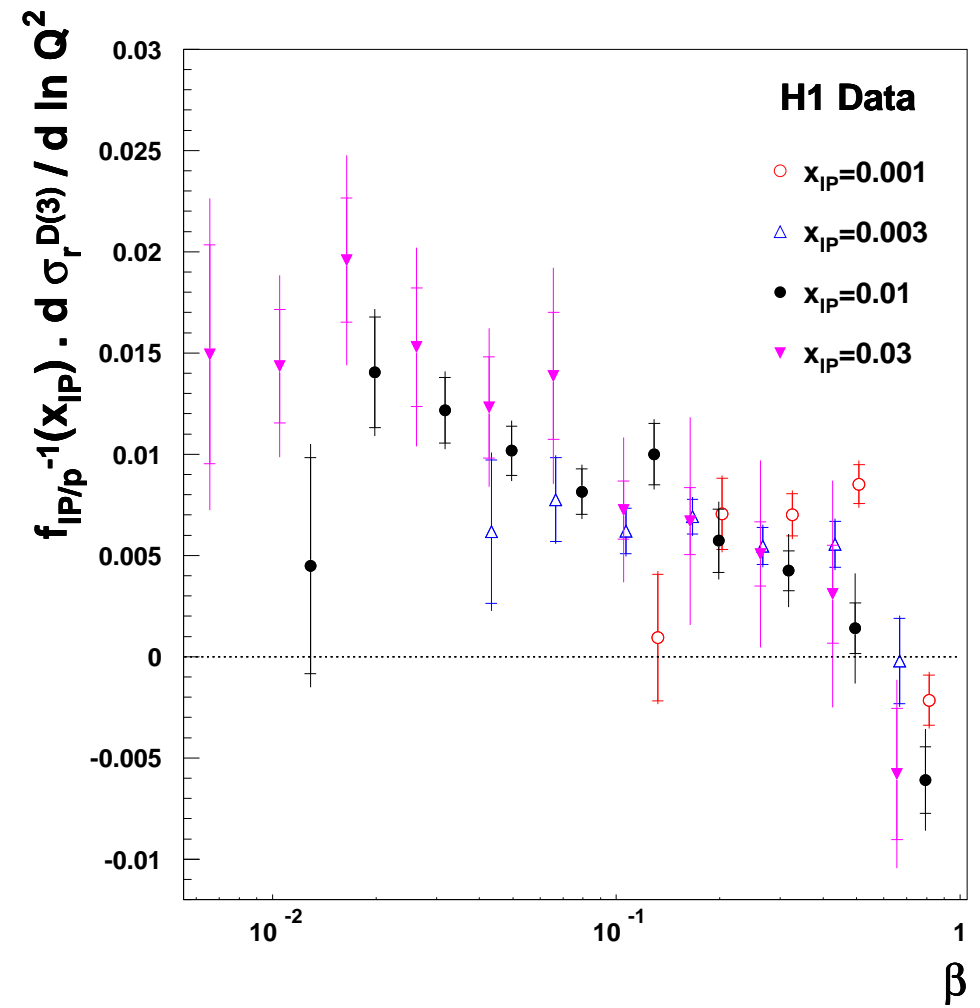

(a)

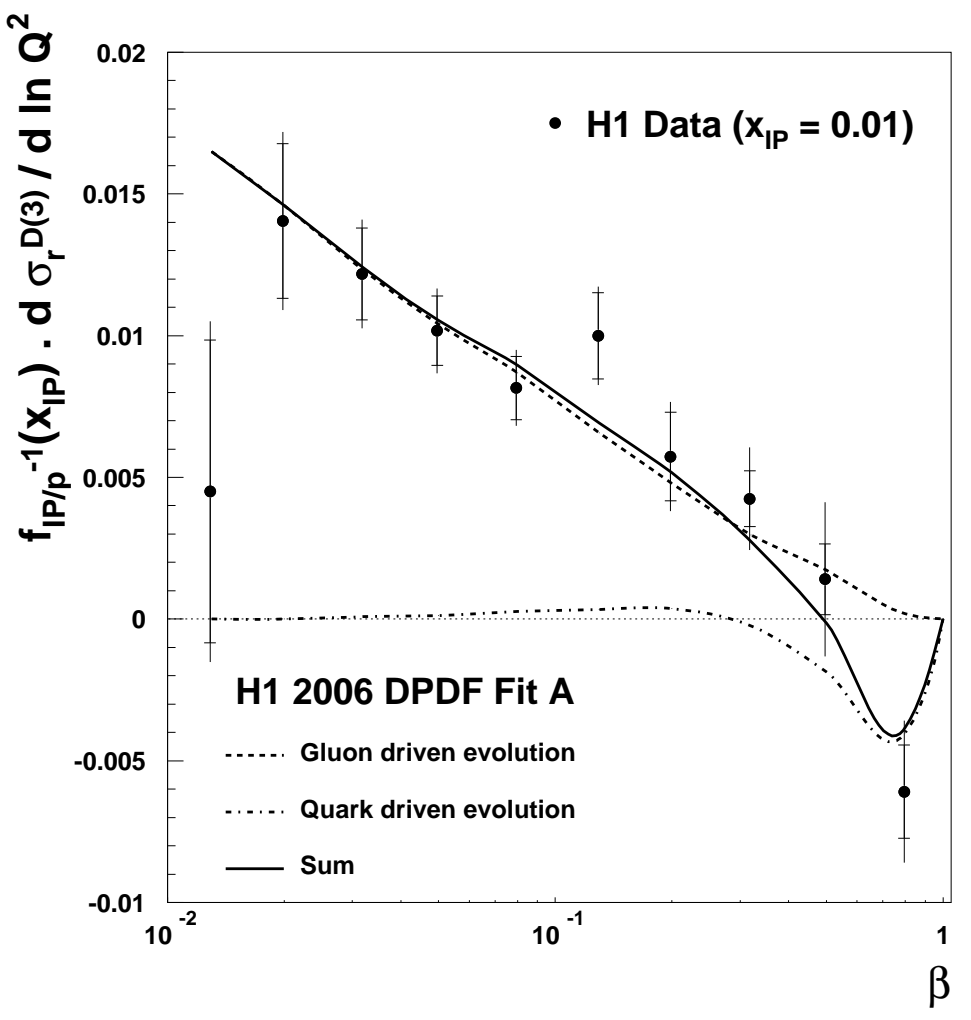

(b)

Figure 7: (a) Measurements of the logarithmic $Q^{2}$ derivative of the reduced diffractive cross section at different fixed values of $x_{I P}$ and $\beta$, obtained by fitting equation 10 to the data. The derivatives are divided by the diffractive flux factor as defined in equation 14. (b) The logarithmic $Q^{2}$ derivative at $x_{\mathbb{P}}=0.01$, divided by the diffractive flux factor and compared with the prediction of the 'H1 2006 DPDF Fit A'. The prediction is also decomposed into contributions to the evolution from the splittings $g \rightarrow q \bar{q}$ ('Gluon driven evolution') and $q \rightarrow q g$ ('Quark driven evolution'). The inner and outer error bars represent the statistical and total uncertainties, respectively. Normalisation uncertainties are not shown. 


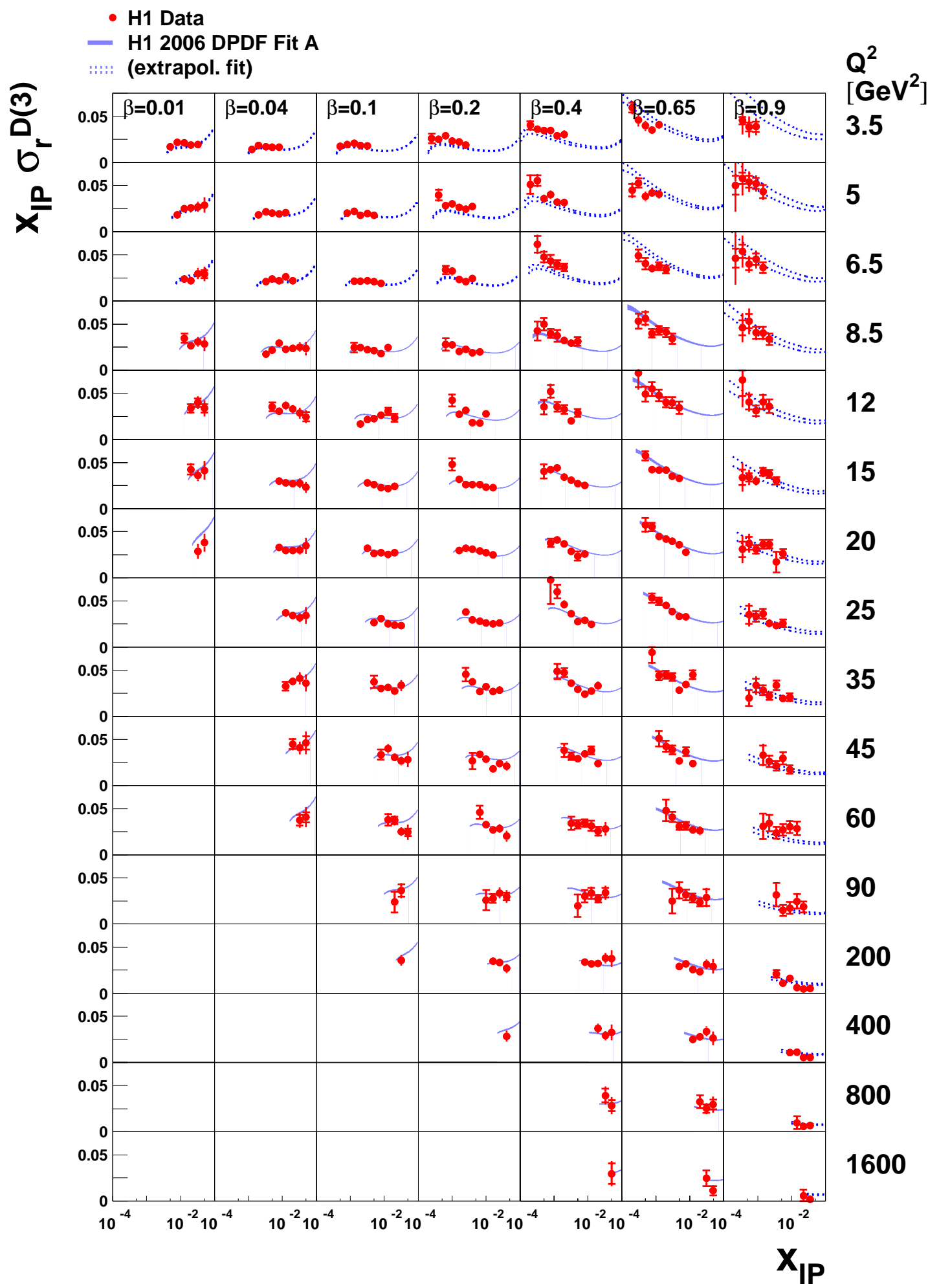

Figure 8: The $x_{\mathbb{P}}$ dependence of the diffractive reduced cross section, multiplied by $x_{\mathbb{P}}$, at fixed values of $\beta$ and $Q^{2}$. The inner and outer error bars on the data points represent the statistical and total uncertainties, respectively. Normalisation uncertainties are not shown. The data are compared with the results of the 'H1 2006 DPDF Fit A' for $E_{p}=820 \mathrm{GeV}$, which is shown as a shaded error band (experimental uncertainties only) in kinematic regions which are included in the fit and as a pair of dashed lines in regions which are excluded from the fit. 


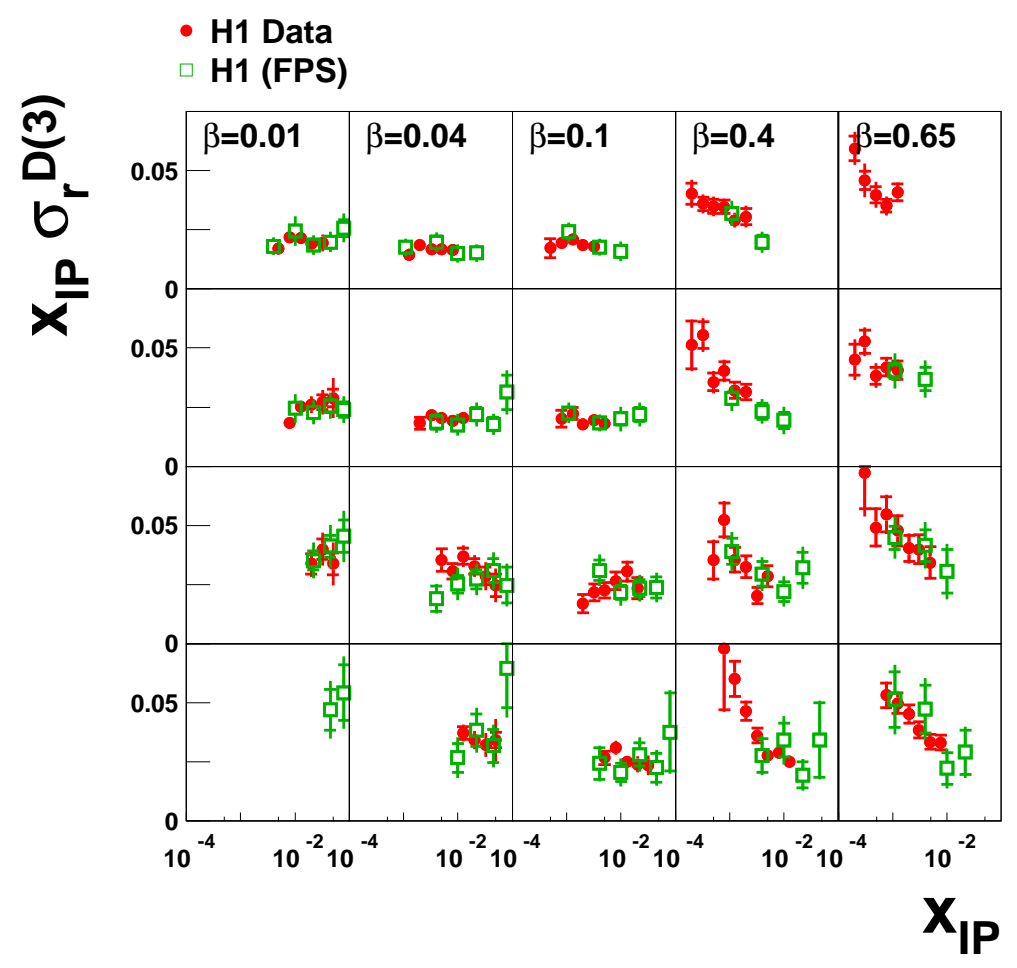

$Q^{2}$

[ $\mathrm{GeV}^{2}$ ]

3.5

5

12

(a)

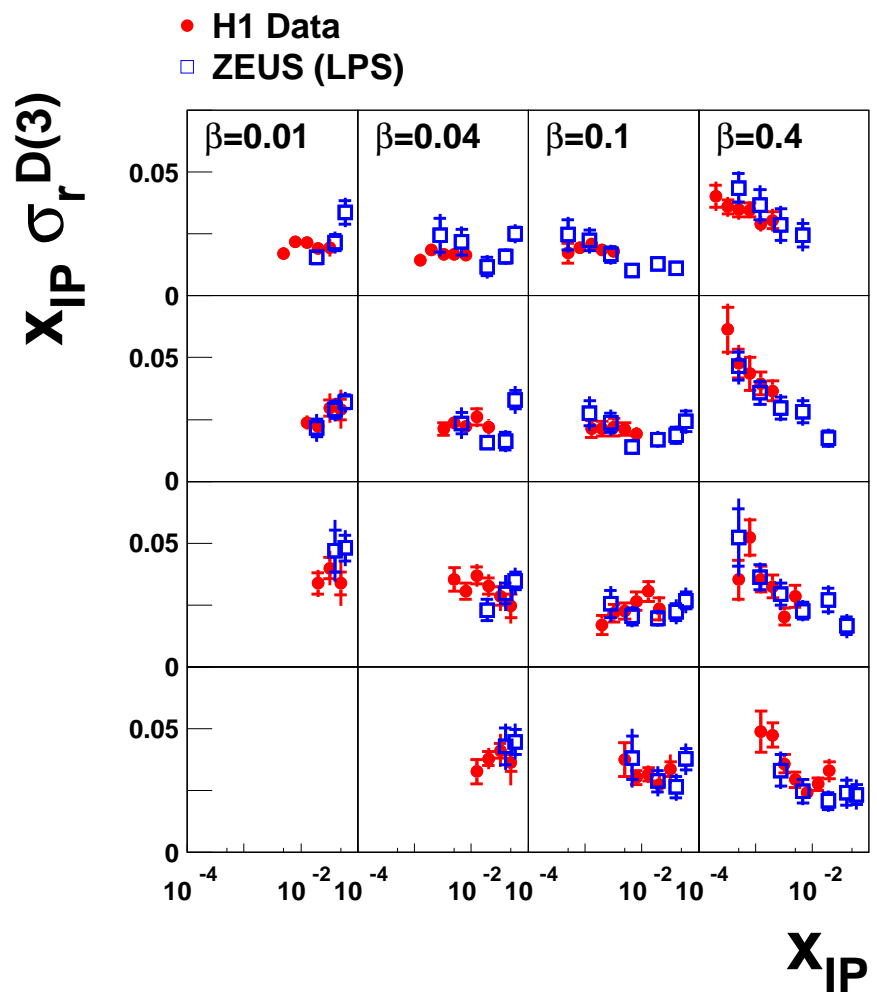

$Q^{2}$

$\left[\mathrm{GeV}^{2}\right]$

3.5

6.5

(b)

12

35

Figure 9: Comparisons between subsets of the present data and results obtained by the direct measurement of the final state proton using (a) the H1 Forward Proton Spectrometer (FPS) [6] and (b) the ZEUS Leading Proton Spectrometer (LPS) [10]. The FPS and LPS data are shifted to the $Q^{2}$ and $\beta$ values shown using small translation factors and are multiplied by a further universal factor of 1.23 such that they correspond to $M_{\mathrm{Y}}<1.6 \mathrm{GeV}$. The inner error bars represent the statistical uncertainties and the outer error bars represent the combined statistical and systematic uncertainties. Normalisation uncertainties are not shown. 


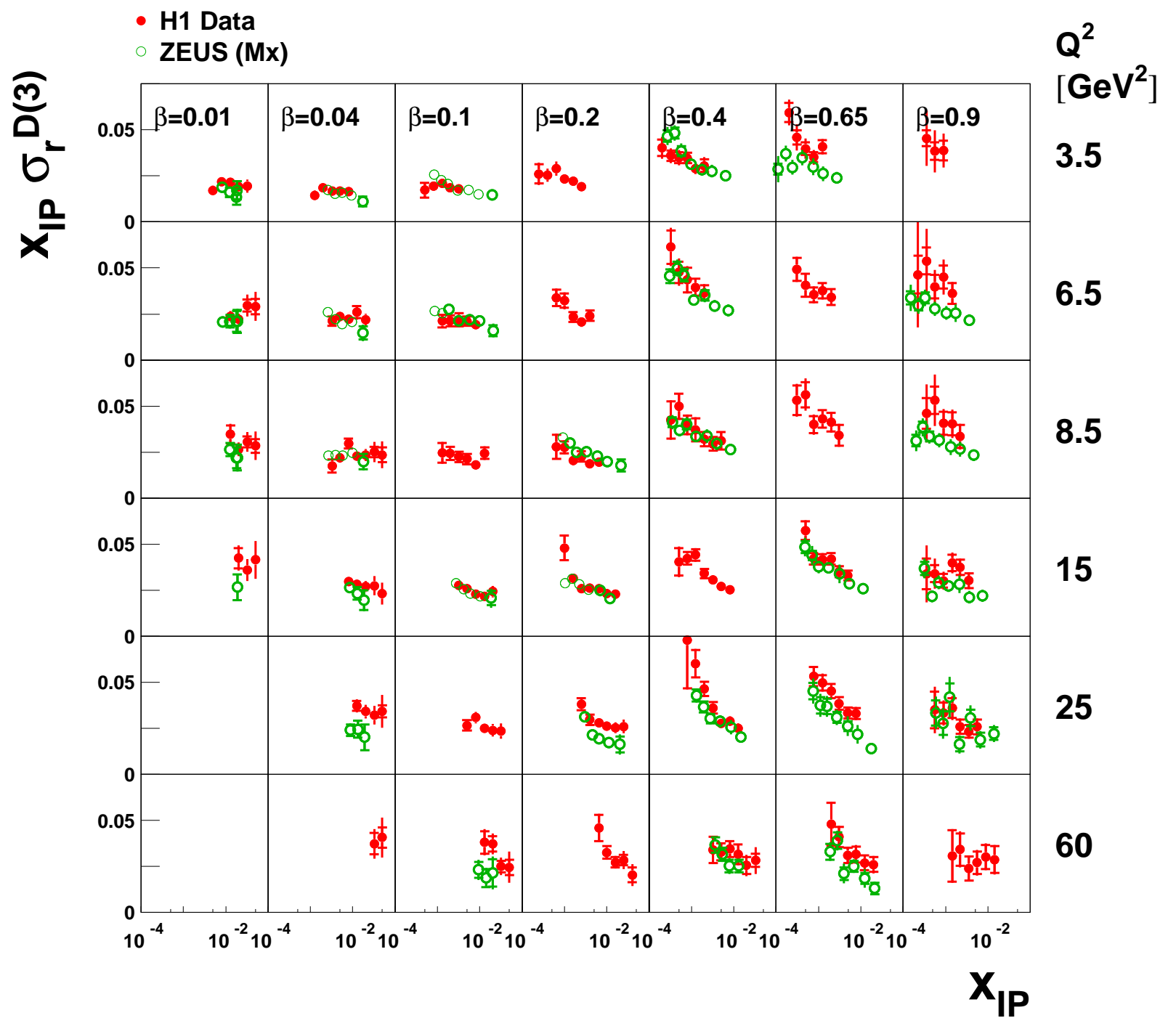

Figure 10: Comparison between a subset of the present data and the results of the ZEUS collaboration, obtained by decomposition of the inclusive $\ln M_{\mathrm{x}}^{2}$ distribution [11]. The ZEUS data are shifted to the $Q^{2}$ and $\beta$ values shown using small translation factors and have been multiplied by a further universal factor of 0.86 so that they correspond to $M_{\mathrm{Y}}<1.6 \mathrm{GeV}$. The inner error bars represent the statistical uncertainties and the outer error bars represent the combined statistical and systematic uncertainties. Normalisation uncertainties are not shown. 

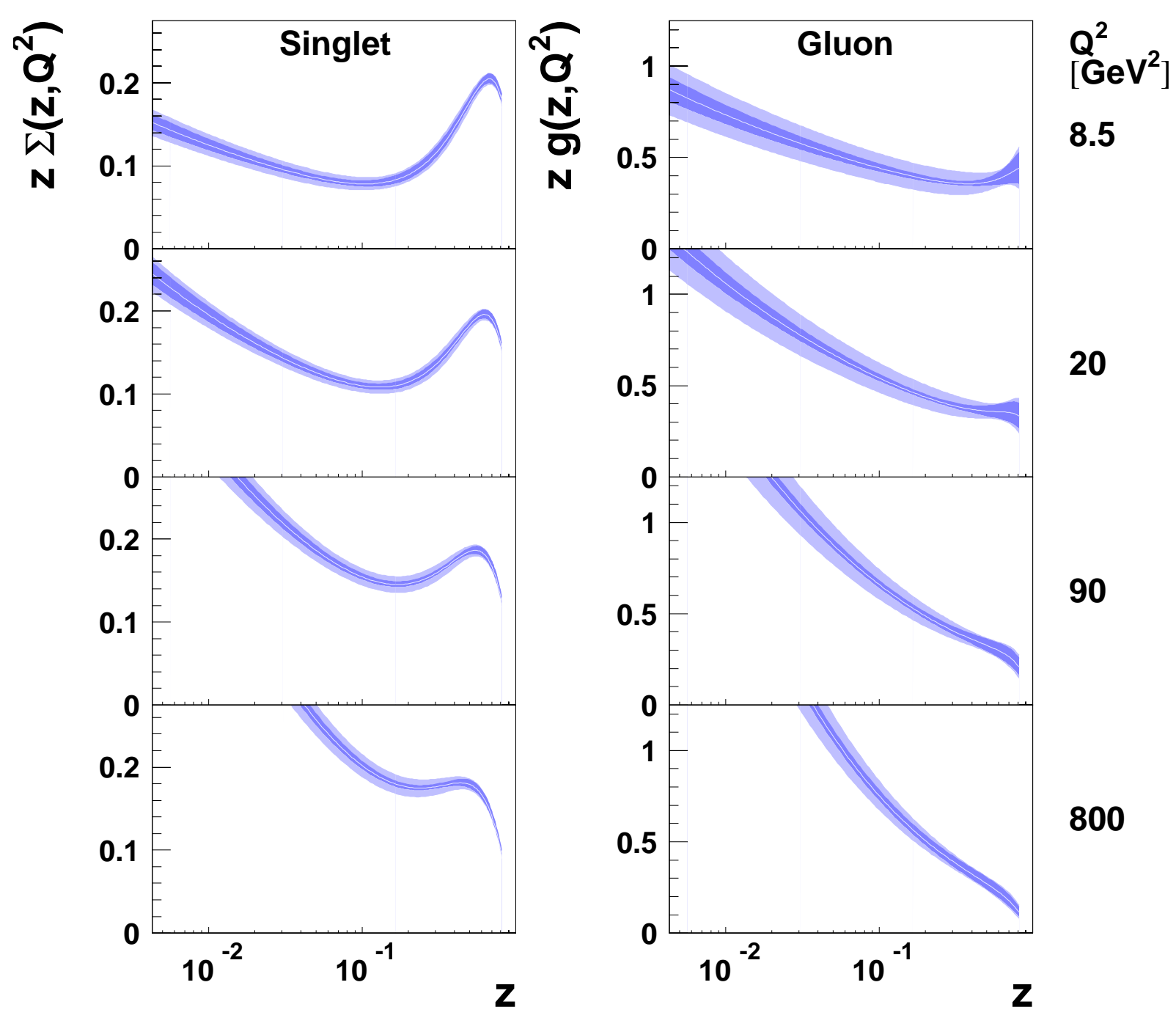

H1 2006 DPDF Fit A

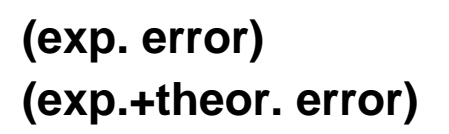

Figure 11: The total quark singlet and gluon distributions obtained from the NLO QCD 'H1 2006 DPDF Fit A', shown at four different values of $Q^{2}$ for the range $0.0043<z<0.8$, corresponding approximately to that of the measurement. The light coloured central lines are surrounded by inner error bands corresponding to the experimental uncertainties and outer error bands corresponding to the experimental and theoretical uncertainties added in quadrature. 


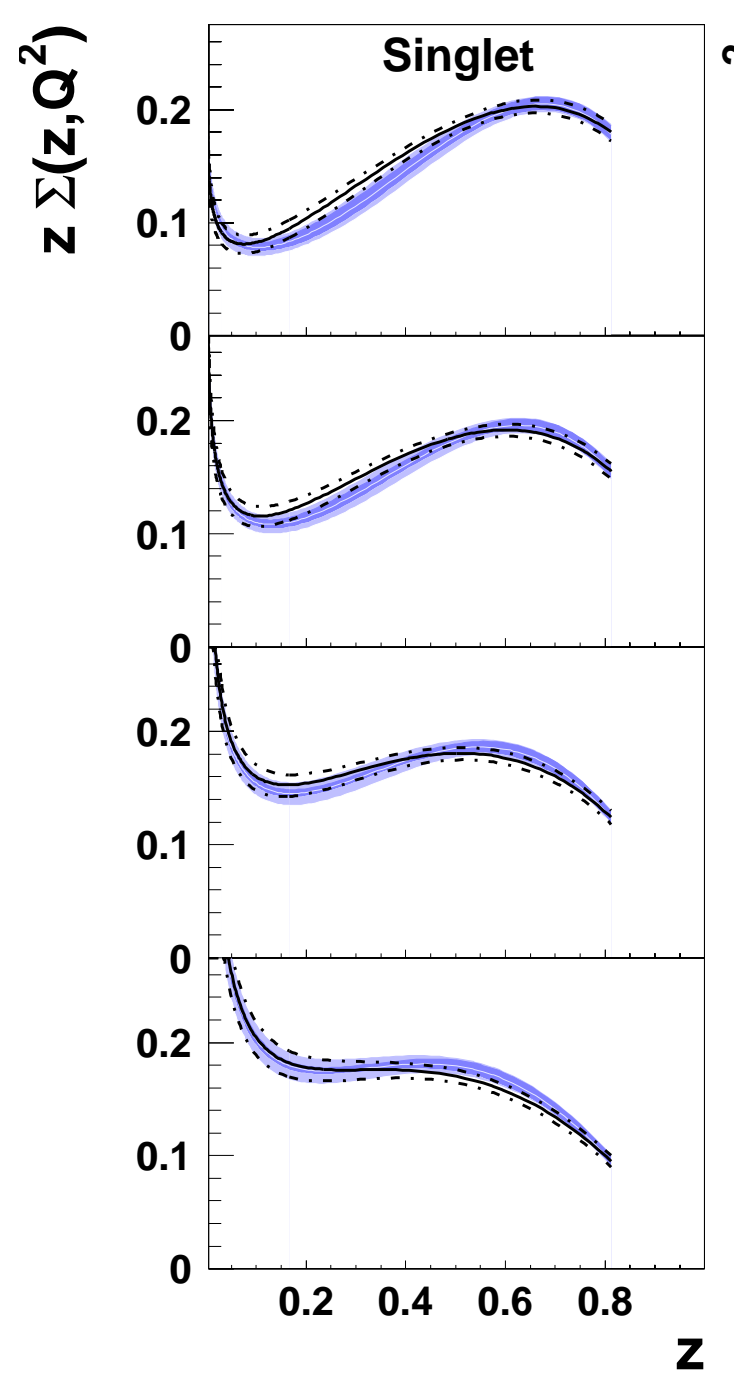

H1 2006 DPDF Fit A

(exp. error)

(exp.+theor. error)
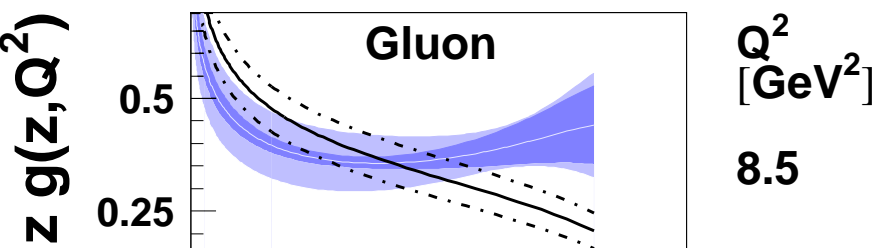

8.5

20

90

800

Figure 12: Comparison on a linear $z$ scale between the total quark singlet and gluon distributions obtained from the 'H1 2006 DPDF Fit A' and the 'H1 2006 DPDF Fit B'. These two fits differ in the parameterisation chosen for the gluon density at the starting scale for QCD evolution. The DPDFs are shown at four different values of $Q^{2}$ for the range $0.0043<z<0.8$, corresponding approximately to that of the measurement. For 'Fit A', the central result is shown as a light coloured central line, which is surrounded by inner error bands corresponding to the experimental uncertainties and outer error bands corresponding to the experimental and theoretical uncertainties added in quadrature. For 'Fit B', only the total uncertainty is shown. 


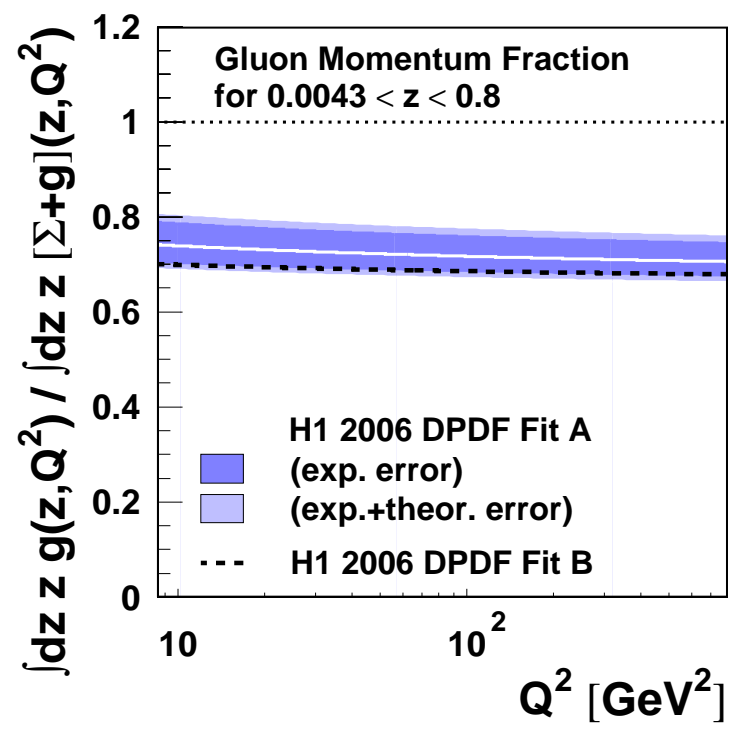

Figure 13: The $Q^{2}$ dependence of the fraction of the longitudinal momentum of the diffractive exchange which is carried by gluons according to the 'H1 2006 DPDF Fit A', integrated over the range $0.0043<z<0.8$, corresponding approximately to that of the measurement. The light coloured central line is surrounded by an inner error band corresponding to the experimental uncertainty and outer error band corresponding to the experimental and theoretical uncertainties added in quadrature. The central result from the 'H1 2006 DPDF Fit B' is also indicated.

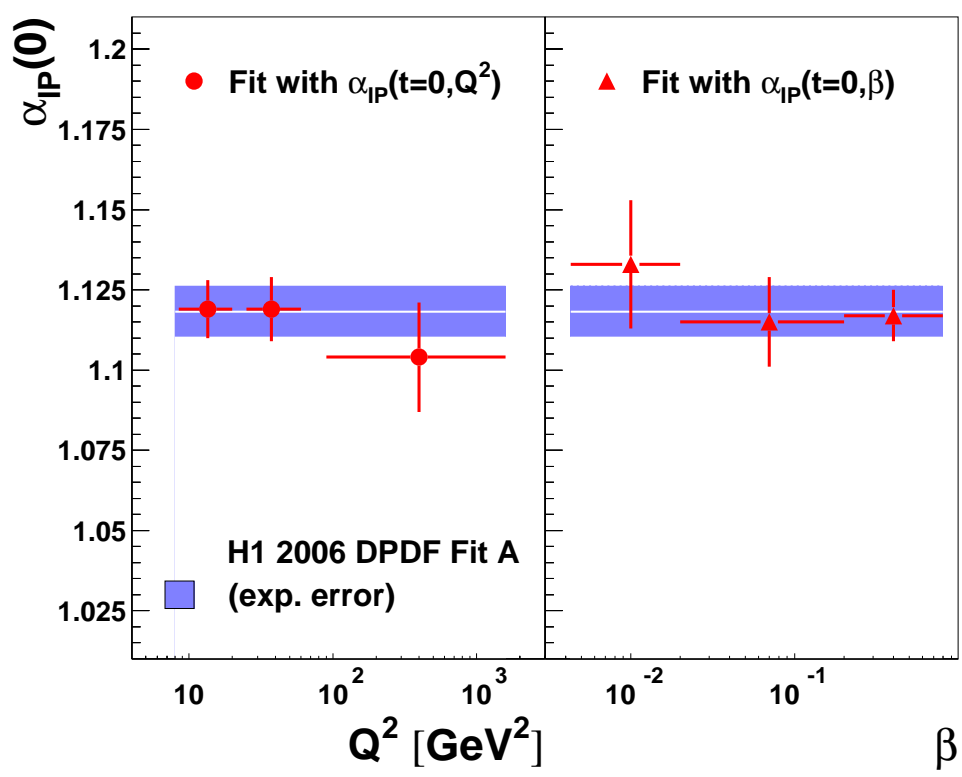

Figure 14: Results from modified versions of the 'H1 2006 DPDF Fit A' in which additional free parameters are included, corresponding to the value of $\alpha_{\mathbb{P}}(0)$ in a number of different ranges of $Q^{2}$ (left) or $\beta$ (right). The data points correspond to the results for $\alpha_{\mathbb{P}}(0)$ in each interval of $Q^{2}$ or $\beta$, with error bars corresponding to the full experimental uncertainties. The bands show the result and experimental uncertainty from the standard fit in which a single parameter is used for $\alpha_{\mathbb{P}}(0)$. 

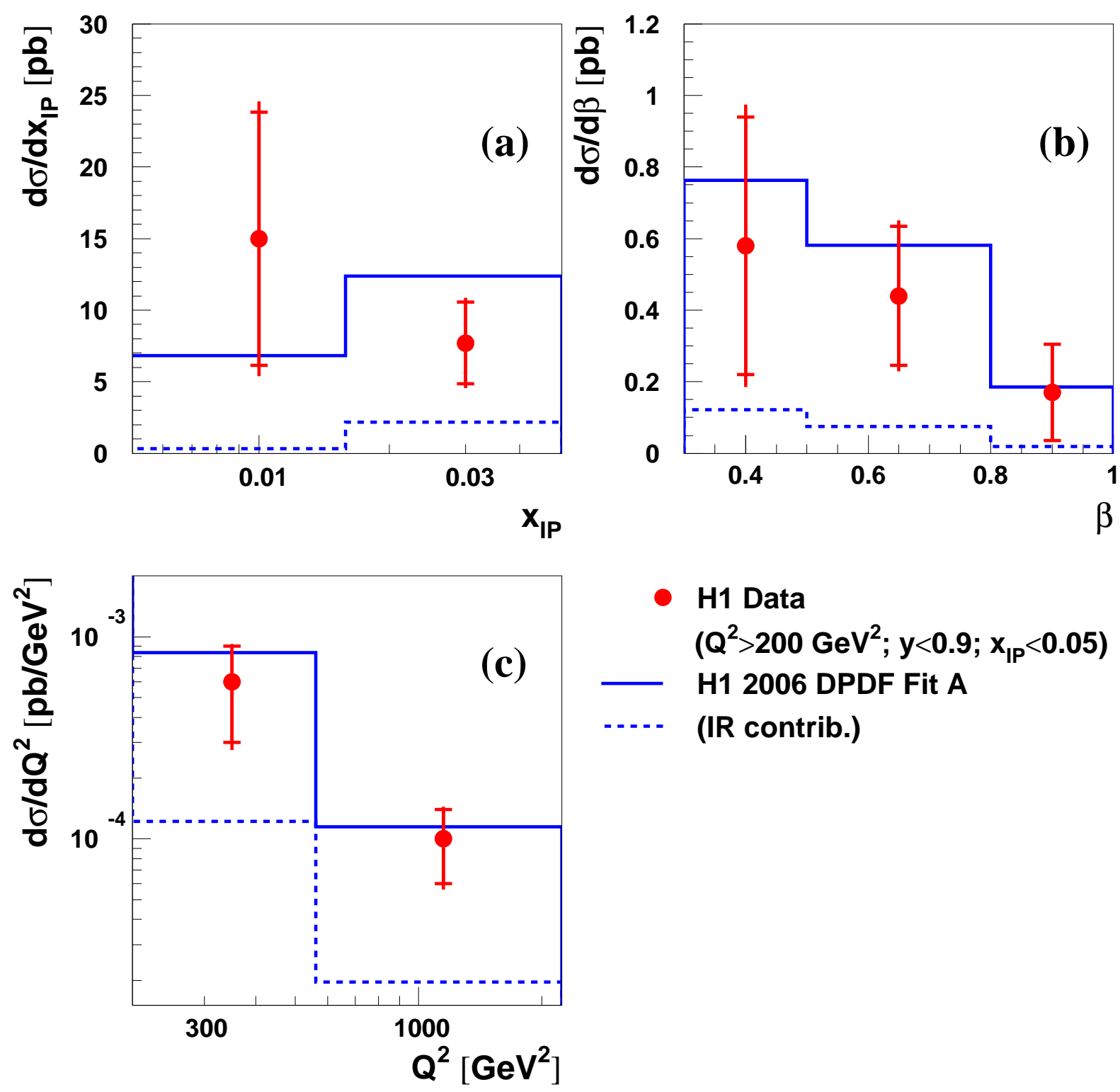

- H1 Data $\left(Q^{2}>200 \mathrm{GeV}^{2} ; \mathrm{y}<0.9 ; \mathrm{x}_{1 \mathrm{P}}<0.05\right)$

H1 2006 DPDF Fit A

-.... (IR contrib.)

Figure 15: The cross section for the diffractive process $e^{+} p \rightarrow \bar{\nu}_{e} X Y$ at $E_{p}=920 \mathrm{GeV}$, shown differentially in (a) $x_{\mathbb{P}}$, (b) $\beta$ and (c) $Q^{2}$, for $Q^{2}>200 \mathrm{GeV}^{2}, y<0.9$ and $x_{\mathbb{P}}<0.05$. The data are compared with the predictions of the 'H1 2006 DPDF Fit A' to the neutral current data, obtained using the RAPGAP Monte Carlo generator. The contribution to the predictions from the sub-leading exchange is also shown. The data points correspond to average values of the differential cross sections over the regions shown by the histograms. The inner and outer error bars on the data points represent the statistical and total uncertainties, respectively. Normalisation uncertainties are not shown. 

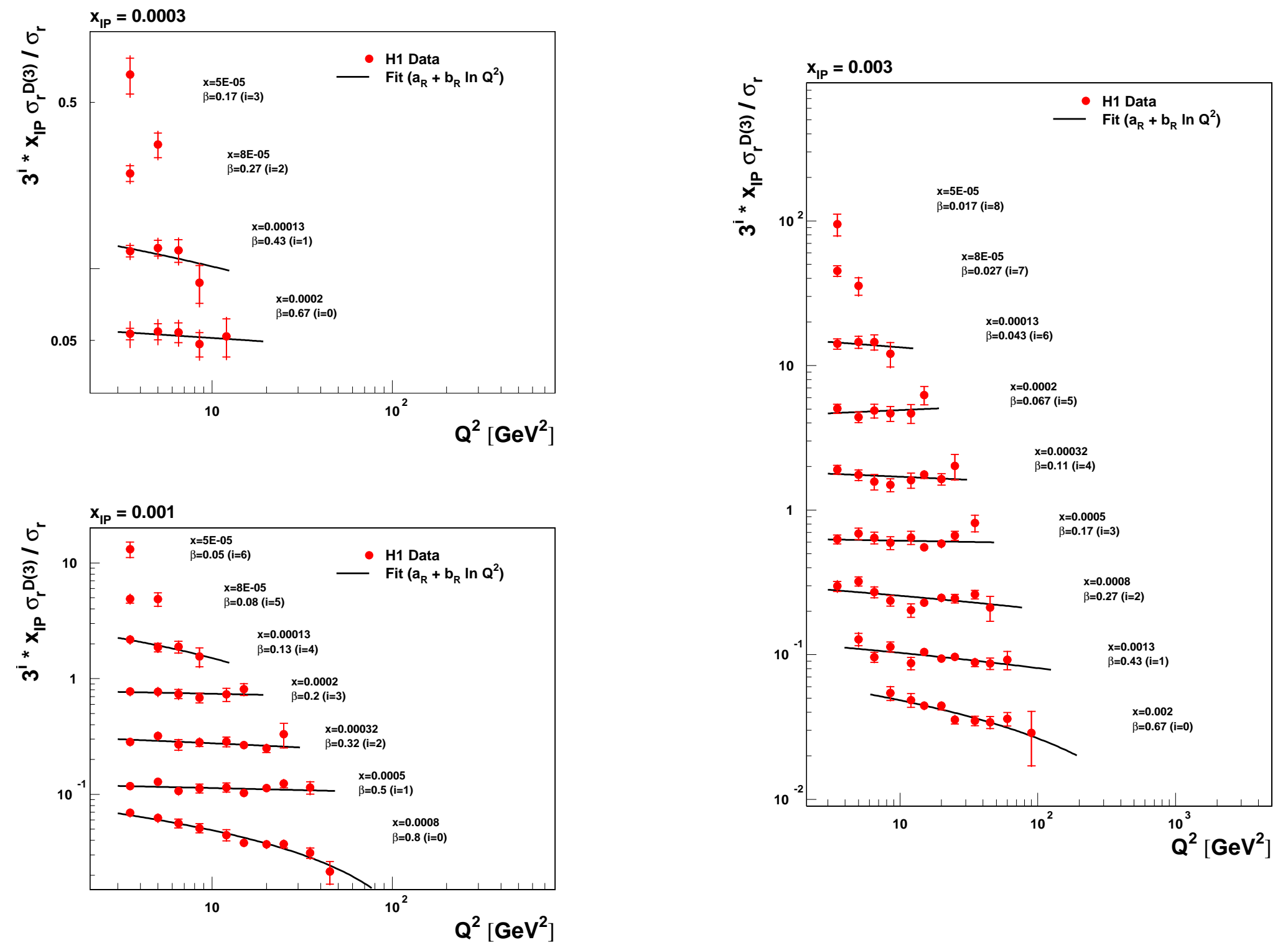

Figure 16: The ratio of the diffractive to the inclusive reduced cross section, multiplied by $x_{\mathbb{P}}$ and shown as a function of $Q^{2}$ for fixed $x$ and fixed $x_{P}=0.0003,0.001$ and 0.003 . The data are multiplied by a further factor of $3^{i}$ for visibility, with $i$ as indicated. The inner and outer error bars represent the statistical and total uncertainties, respectively. Normalisation uncertainties are not shown. The results of fits of a linear dependence on $\log Q^{2}$ (equation 19) to the data are also shown. 

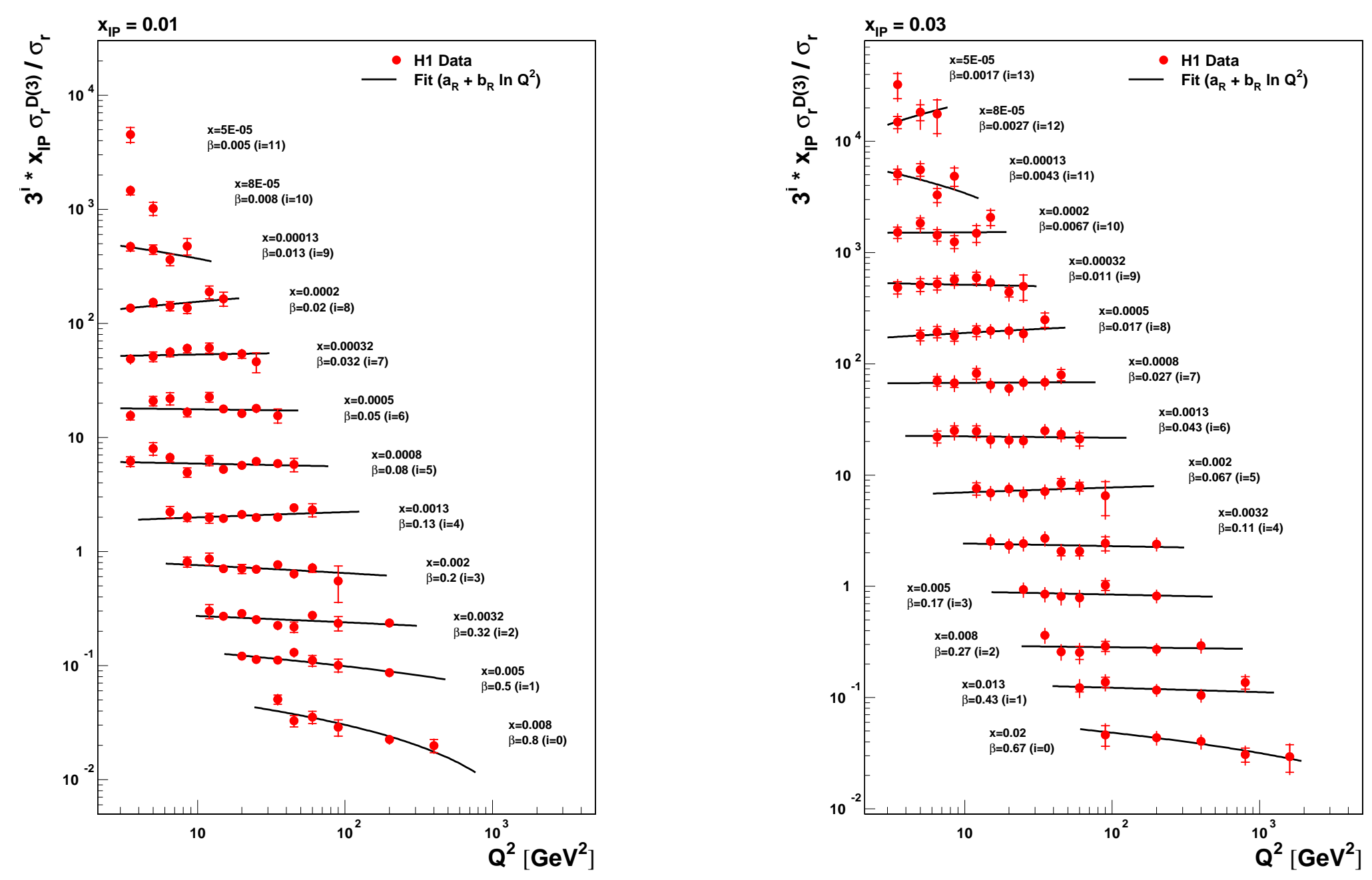

Figure 17: The ratio of the diffractive to the inclusive reduced cross section, multiplied by $x_{\mathbb{P}}$ and shown as a function of $Q^{2}$ for fixed $x$ and fixed $x_{\mathbb{P}}=0.01$ and 0.03 . See the caption of figure 16 for further details. 


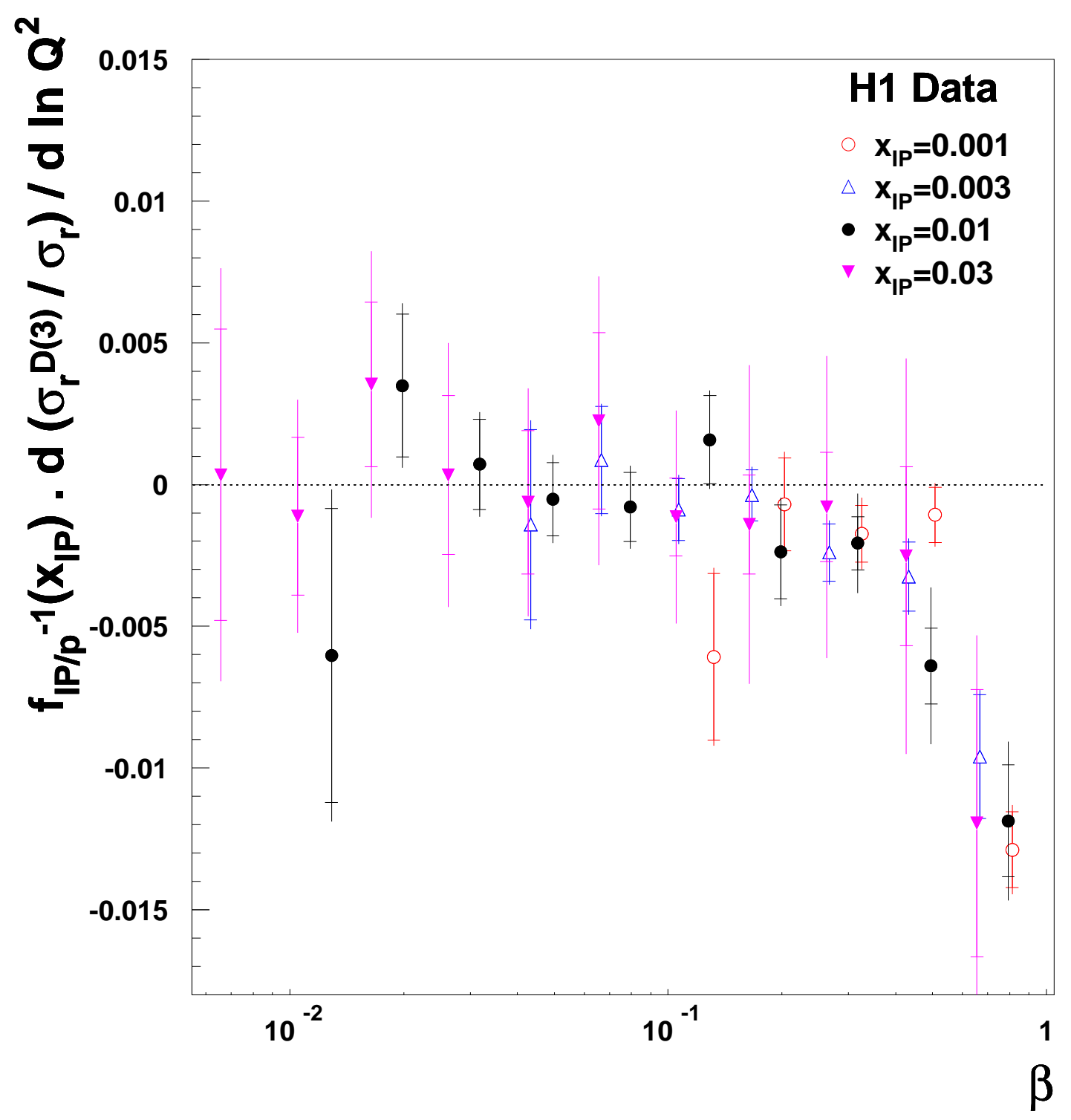

Figure 18: The logarithmic $Q^{2}$ derivative of the ratio of the reduced diffractive cross section to the reduced inclusive cross section at different fixed values of $x_{I P}$ and $\beta$ (see equation 19). The derivatives are divided by the diffractive flux factor as defined in equation 14 . The inner and outer error bars represent the statistical and total uncertainties, respectively. Normalisation uncertainties are not shown. 


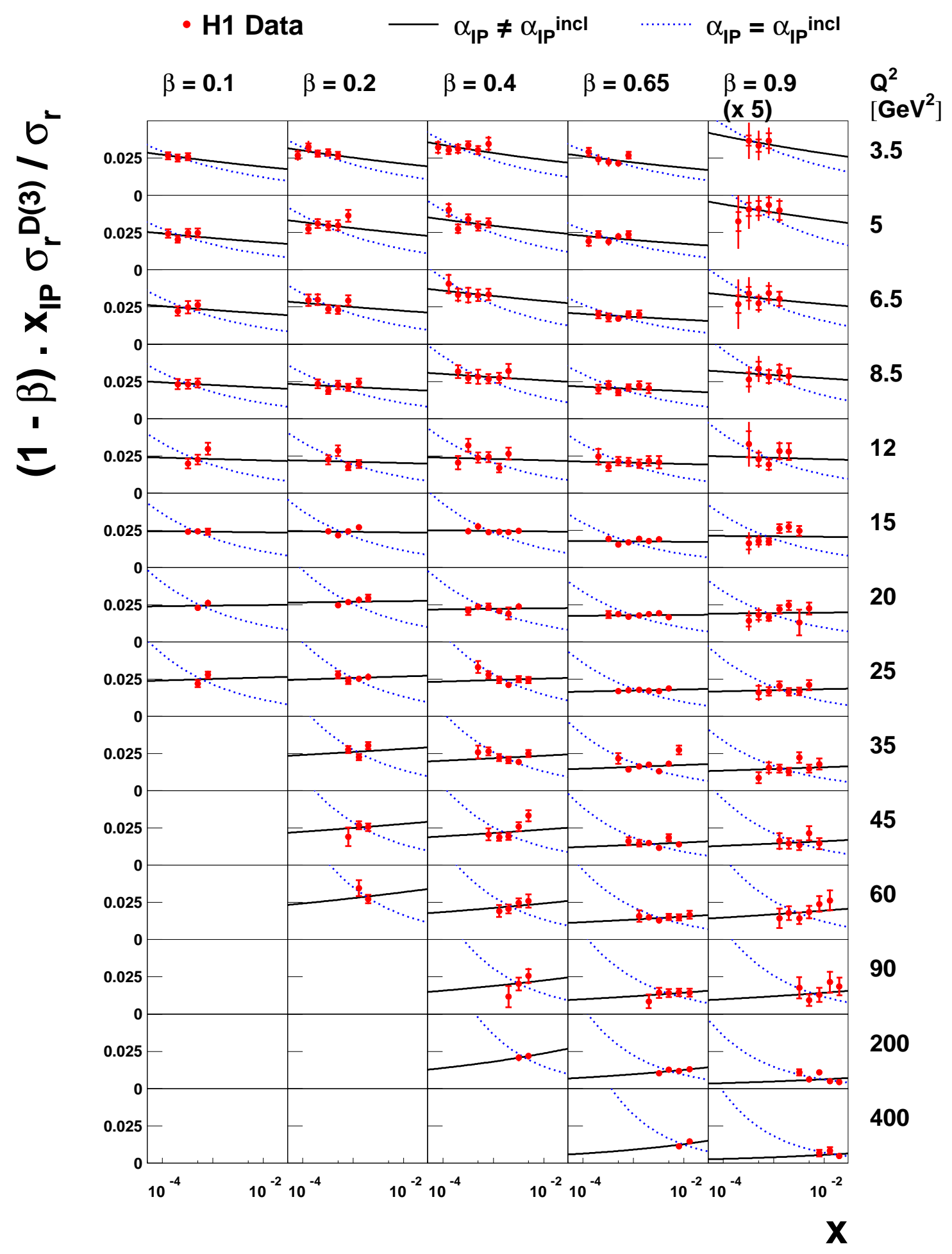

Figure 19: The ratio of the diffractive to the inclusive reduced cross section, multiplied by $(1-\beta) x_{\mathbb{P}}$. Data at $\beta=0.9$ are multiplied by a further factor of 5 for visibility. Data which are significantly influenced by the sub-leading exchange or the longitudinal structure function according to the NLO QCD fit are excluded. The remaining data are compared with models motivated by Regge phenomenology as described in the text, for which the diffractive and inclusive effective pomeron trajectories are the same (' $\alpha_{\mathbb{P}}=\alpha_{\mathbb{P}}^{\text {incl }}$ ) $)$ and for which they are different ( ${ }^{\prime} \alpha_{\mathbb{P}} \neq \alpha_{\mathbb{P}}^{\text {incl }}$ '). 


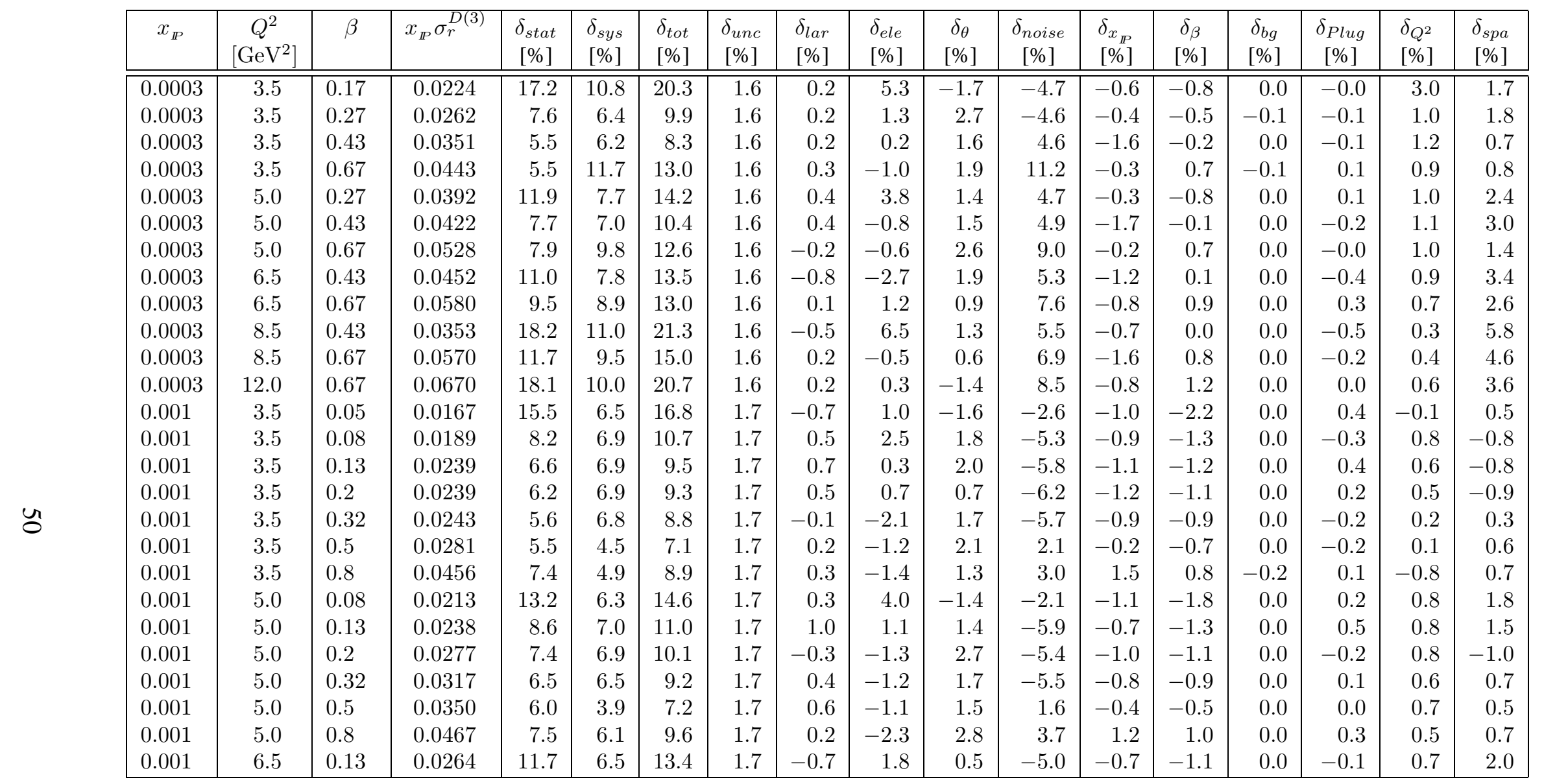

Table 4: Results for $x_{\mathbb{P}} \sigma_{r}^{D(3)}$ at fixed $Q^{2}, \beta$ and $x_{\mathbb{P}}$ (columns 1-4) using data with SPACAL electrons and $E_{p}=820 \mathrm{GeV}$. Columns 5-7 contain the percentage statistical, systematic and total uncertainties. The remaining columns contain the contributions to the systematic uncertainty from sources which are uncorrelated between data points $\left(\delta_{u n c}\right)$ and the 10 correlated sources leading to the largest uncertainties. These are the LAr hadronic energy scale $\left(\delta_{\text {lar }}\right)$, the SPACAL electromagnetic energy scale $\left(\delta_{\text {ele }}\right)$, the scattered electron angle measurement $\left(\delta_{\theta}\right)$, the calorimeter noise treatment $\left(\delta_{\text {noise }}\right)$, reweighting the simulation in $x_{\mathbb{P}}\left(\delta_{x_{\mathbb{P}}}\right)$ and $\beta\left(\delta_{\beta}\right)$, the background subtraction using DJANGO $\left(\delta_{b g}\right)$, the plug energy scale $\left(\delta_{\text {Plug }}\right)$, reweighting the simulation in $Q^{2}\left(\delta_{Q^{2}}\right)$ and the SPACAL hadronic energy scale $\left(\delta_{\text {spa }}\right)$. Minus signs appear for these systematics if the shift in a variable is anti-correlated rather than correlated with the shift in the cross section. The table continues on the next 7 pages. 


\begin{tabular}{|c|c|c|c|c|c|c|c|c|c|c|c|c|c|c|c|c|c|}
\hline$x_{\mathbb{P}}$ & $\begin{array}{c}Q^{2} \\
{\left[\mathrm{GeV}^{2}\right]}\end{array}$ & $\beta$ & $x_{\mathbb{P}} \sigma_{r}^{D(3)}$ & $\begin{array}{c}\delta_{\text {stat }} \\
{[\%]} \\
\end{array}$ & $\begin{array}{l}\delta_{\text {sys }} \\
{[\%]} \\
\end{array}$ & $\begin{array}{l}\delta_{t o t} \\
{[\%]}\end{array}$ & $\begin{array}{c}\delta_{u n c} \\
{[\%]}\end{array}$ & $\begin{array}{l}\delta_{\text {lar }} \\
{[\%]}\end{array}$ & $\begin{array}{l}\delta_{\text {ele }} \\
{[\%]}\end{array}$ & $\begin{array}{c}\delta_{\theta} \\
{[\%]}\end{array}$ & $\begin{array}{c}\delta_{\text {noise }} \\
{[\%]}\end{array}$ & $\begin{array}{l}\delta_{x_{\mathbb{P}}} \\
{[\%]} \\
\end{array}$ & $\begin{array}{c}\delta_{\beta} \\
{[\%]}\end{array}$ & $\begin{array}{c}\delta_{b g} \\
{[\%]}\end{array}$ & $\begin{array}{c}\delta_{\text {Plug }} \\
{[\%]}\end{array}$ & $\begin{array}{l}\delta_{Q^{2}} \\
{[\%]}\end{array}$ & $\begin{array}{l}\delta_{\text {spa }} \\
{[\%]} \\
\end{array}$ \\
\hline 0.001 & 6.5 & 0.2 & 0.0294 & 9.2 & 7.2 & 11.7 & 1.7 & 0.2 & -1.7 & 1.5 & -6.1 & -1.1 & -1.3 & 0.0 & 0.1 & 0.4 & 1.1 \\
\hline 0.001 & 6.5 & 0.32 & 0.0295 & 10.6 & 6.3 & 12.4 & 1.7 & 0.3 & 0.8 & 1.6 & -5.4 & -0.9 & -1.0 & 0.0 & 0.3 & 0.3 & 0.9 \\
\hline 0.001 & 6.5 & 0.5 & 0.0321 & 7.5 & 4.6 & 8.8 & 1.7 & 0.5 & -1.1 & 1.2 & 2.9 & -0.7 & -0.5 & 0.0 & 0.2 & 0.3 & 0.7 \\
\hline 0.001 & 6.5 & 0.8 & 0.0461 & 8.7 & 5.3 & 10.2 & 1.7 & 0.8 & -1.7 & 1.4 & 3.8 & 0.6 & 0.9 & 0.0 & -0.2 & 0.3 & 0.9 \\
\hline 0.001 & 8.5 & 0.13 & 0.0232 & 18.6 & 8.6 & 20.5 & 1.7 & 0.9 & -3.0 & 2.6 & -6.5 & -0.8 & -1.6 & 0.0 & -0.2 & 0.6 & 2.4 \\
\hline 0.001 & 8.5 & 0.2 & 0.0298 & 9.6 & 5.2 & 10.9 & 1.7 & -0.6 & 1.2 & 1.0 & -4.0 & -0.6 & -1.2 & 0.0 & -0.2 & 0.2 & -1.3 \\
\hline 0.001 & 8.5 & 0.32 & 0.0341 & 8.4 & 5.6 & 10.1 & 1.7 & 0.5 & -1.7 & 2.4 & -4.0 & -0.7 & -0.9 & 0.0 & 0.3 & 0.2 & -0.6 \\
\hline 0.001 & 8.5 & 0.5 & 0.0372 & 8.8 & 5.1 & 10.2 & 1.7 & 0.3 & -2.8 & 1.9 & -2.4 & -0.5 & -0.5 & 0.0 & 0.1 & 0.2 & 0.8 \\
\hline 0.001 & 8.5 & 0.8 & 0.0457 & 9.1 & 4.8 & 10.2 & 1.7 & -0.3 & -1.3 & 2.6 & 2.4 & 0.9 & 1.0 & 0.0 & -0.0 & 0.1 & 0.6 \\
\hline 0.001 & 12.0 & 0.2 & 0.0349 & 13.0 & 6.0 & 14.3 & 1.7 & 1.4 & -1.7 & 1.1 & -2.9 & -0.5 & -1.3 & 0.0 & 0.6 & 0.7 & 3.8 \\
\hline 0.001 & 12.0 & 0.32 & 0.0385 & 9.6 & 5.6 & 11.1 & 1.7 & 0.6 & -1.7 & 1.2 & -3.8 & -0.8 & -1.0 & 0.0 & -0.2 & 0.6 & -0.3 \\
\hline 0.001 & 12.0 & 0.5 & 0.0426 & 9.0 & 5.4 & 10.5 & 1.7 & 0.5 & -1.0 & 2.9 & 3.3 & -0.3 & -0.4 & 0.0 & 0.2 & 0.5 & 1.2 \\
\hline 0.001 & 12.0 & 0.8 & 0.0445 & 11.0 & 5.6 & 12.4 & 1.7 & 0.3 & -2.0 & -0.7 & 3.7 & 1.1 & 0.9 & 0.0 & 0.2 & 0.4 & 1.2 \\
\hline 0.001 & 15.0 & 0.2 & 0.0395 & 11.6 & 6.3 & 13.2 & 1.7 & 0.3 & 4.0 & -0.9 & -3.3 & -0.3 & -1.9 & 0.0 & 0.2 & 0.1 & 1.9 \\
\hline 0.001 & 15.0 & 0.32 & 0.0384 & 5.3 & 4.5 & 7.0 & 1.7 & 0.4 & 0.7 & 0.8 & -3.4 & -0.6 & -1.0 & 0.0 & -0.1 & 0.1 & 1.3 \\
\hline 0.001 & 15.0 & 0.5 & 0.0410 & 4.9 & 5.3 & 7.2 & 1.7 & 0.5 & -3.1 & 1.7 & 2.9 & -0.3 & -0.5 & 0.0 & 0.0 & 0.0 & 0.5 \\
\hline 0.001 & 15.0 & 0.8 & 0.0409 & 6.4 & 4.7 & 7.9 & 1.7 & 0.1 & -2.1 & 1.3 & 2.6 & 0.8 & 1.0 & 0.0 & -0.1 & -0.2 & 0.7 \\
\hline 0.001 & 20.0 & 0.32 & 0.0379 & 8.0 & 4.9 & 9.4 & 1.7 & 0.4 & 1.1 & 0.5 & -2.8 & -0.4 & -1.1 & -0.4 & -0.1 & 0.4 & 2.7 \\
\hline 0.001 & 20.0 & 0.5 & 0.0488 & 5.5 & 4.4 & 7.0 & 1.7 & 0.5 & -2.3 & 0.4 & 2.3 & -0.3 & -0.3 & 0.0 & 0.1 & 0.3 & 1.0 \\
\hline 0.001 & 20.0 & 0.8 & 0.0431 & 6.9 & 5.8 & 9.0 & 1.7 & 0.3 & -2.9 & 1.5 & 3.3 & 0.9 & 1.0 & 0.0 & 0.1 & 0.2 & 1.1 \\
\hline 0.001 & 25.0 & 0.32 & 0.0505 & 23.9 & 6.9 & 24.8 & 1.7 & 0.5 & 4.6 & 1.9 & -1.5 & -0.6 & -1.4 & 0.0 & -0.6 & 0.1 & 3.3 \\
\hline 0.001 & 25.0 & 0.5 & 0.0562 & 8.4 & 4.6 & 9.6 & 1.7 & 0.5 & -1.8 & 1.0 & 0.9 & 0.1 & -0.4 & 0.0 & 0.2 & 0.1 & 2.8 \\
\hline 0.001 & 25.0 & 0.8 & 0.0460 & 7.8 & 5.9 & 9.8 & 1.7 & 0.8 & -2.3 & 1.1 & 3.5 & 0.3 & 1.1 & -0.3 & -0.2 & -0.2 & 1.2 \\
\hline 0.001 & 35.0 & 0.5 & 0.0534 & 12.4 & 7.1 & 14.3 & 1.7 & 0.6 & 2.2 & 1.0 & 2.4 & -0.5 & -0.5 & 0.0 & 0.7 & 0.5 & 5.3 \\
\hline 0.001 & 35.0 & 0.8 & 0.0416 & 10.4 & 7.0 & 12.6 & 1.7 & 0.3 & -4.0 & 1.0 & 2.9 & 0.8 & 1.0 & 0.0 & 0.0 & 0.2 & 2.1 \\
\hline 0.001 & 45.0 & 0.8 & 0.0297 & 22.1 & 8.8 & 23.8 & 1.7 & 0.5 & 2.1 & 1.8 & 3.9 & 1.3 & 0.8 & 0.0 & 0.0 & 0.3 & 4.5 \\
\hline 0.003 & 3.5 & 0.017 & 0.0134 & 17.2 & 6.8 & 18.5 & 2.1 & 0.5 & 2.9 & 0.7 & 0.4 & -0.4 & -1.3 & 0.0 & -0.3 & 0.5 & 0.8 \\
\hline 0.003 & 3.5 & 0.027 & 0.0194 & 8.4 & 4.6 & 9.5 & 2.1 & 0.7 & 1.4 & 1.5 & -2.2 & -0.6 & -1.2 & -0.2 & -0.1 & 0.8 & 0.6 \\
\hline 0.003 & 3.5 & 0.043 & 0.0172 & 8.1 & 4.0 & 9.0 & 2.1 & 0.1 & 0.6 & 2.8 & -0.9 & -0.2 & -0.5 & -0.2 & 0.1 & 0.6 & 0.9 \\
\hline 0.003 & 3.5 & 0.067 & 0.0172 & 7.4 & 3.7 & 8.3 & 2.1 & 0.3 & 0.7 & 1.2 & -1.1 & -0.9 & -1.0 & -0.2 & -0.3 & 0.5 & -1.0 \\
\hline 0.003 & 3.5 & 0.11 & 0.0181 & 6.9 & 3.6 & 7.8 & 2.1 & 1.0 & -0.2 & 1.1 & -1.7 & -0.8 & -0.9 & -0.2 & 0.2 & 0.2 & -0.3 \\
\hline 0.003 & 3.5 & 0.17 & 0.0166 & 7.1 & 4.3 & 8.3 & 2.1 & 0.7 & -0.8 & 1.2 & -2.2 & -1.0 & -1.0 & -0.3 & 0.2 & 0.3 & 0.5 \\
\hline 0.003 & 3.5 & 0.27 & 0.0218 & 8.0 & 6.1 & 10.1 & 2.1 & 0.7 & -1.0 & 0.8 & -1.5 & -1.1 & -1.2 & -0.3 & 0.1 & -3.3 & -0.8 \\
\hline 0.003 & 5.0 & 0.027 & 0.0173 & 13.9 & 7.5 & 15.8 & 2.1 & -0.3 & 5.3 & 1.2 & -2.7 & -0.5 & -1.3 & -0.3 & 0.2 & 0.5 & -1.4 \\
\hline 0.003 & 5.0 & 0.043 & 0.0207 & 9.6 & 4.9 & 10.7 & 2.1 & 1.3 & 2.8 & 0.8 & -1.8 & -0.6 & -1.1 & -0.3 & 0.4 & 0.9 & 1.1 \\
\hline 0.003 & 5.0 & 0.067 & 0.0175 & 8.4 & 4.2 & 9.4 & 2.1 & 0.7 & 0.9 & 1.7 & -1.9 & -0.6 & -1.1 & -0.4 & -0.2 & 0.7 & 0.9 \\
\hline
\end{tabular}




\begin{tabular}{|c|c|c|c|c|c|c|c|c|c|c|c|c|c|c|c|c|c|}
\hline$x_{\mathbb{P}}$ & $\begin{array}{c}Q^{2} \\
{\left[\mathrm{GeV}^{2}\right]}\end{array}$ & $\beta$ & $x_{\mathbb{I P}} \sigma_{r}^{D(3)}$ & $\begin{array}{c}\delta_{\text {stat }} \\
{[\%]} \\
\end{array}$ & $\begin{array}{l}\delta_{\text {sys }} \\
{[\%]}\end{array}$ & $\begin{array}{l}\delta_{t o t} \\
{[\%]}\end{array}$ & $\begin{array}{c}\delta_{u n c} \\
{[\%]} \\
\end{array}$ & $\begin{array}{l}\delta_{l a r} \\
{[\%]}\end{array}$ & $\begin{array}{l}\delta_{\text {ele }} \\
{[\%]}\end{array}$ & $\begin{array}{c}\delta_{\theta} \\
{[\%]}\end{array}$ & $\begin{array}{c}\delta_{\text {noise }} \\
{[\%]}\end{array}$ & $\begin{array}{l}\delta_{x_{I P}} \\
{[\%]}\end{array}$ & $\begin{array}{c}\delta_{\beta} \\
{[\%]}\end{array}$ & $\begin{array}{c}\delta_{b g} \\
{[\%]}\end{array}$ & $\begin{array}{c}\delta_{\text {Plug }} \\
{[\%]}\end{array}$ & $\begin{array}{l}\delta_{Q^{2}} \\
{[\%]}\end{array}$ & $\begin{array}{l}\delta_{s p a} \\
{[\%]}\end{array}$ \\
\hline 0.003 & 5.0 & 0.11 & 0.0193 & 8.4 & 5.2 & 9.9 & 2.1 & -0.3 & -1.5 & 2.0 & -3.2 & -0.9 & -0.9 & -0.1 & -0.6 & 0.6 & -0.3 \\
\hline 0.003 & 5.0 & 0.17 & 0.0209 & 9.0 & 4.7 & 10.2 & 2.1 & -0.4 & -0.7 & 2.0 & -2.8 & -1.0 & -1.0 & -0.7 & -0.4 & 0.7 & -0.3 \\
\hline 0.003 & 5.0 & 0.27 & 0.0268 & 7.3 & 4.2 & 8.4 & 2.1 & 1.1 & -0.9 & 1.3 & -1.4 & -1.0 & -0.9 & -0.2 & 0.2 & 0.2 & 0.1 \\
\hline 0.003 & 5.0 & 0.43 & 0.0290 & 9.9 & 7.3 & 12.3 & 2.1 & 0.3 & 0.4 & 0.8 & -1.1 & -0.9 & -0.7 & 0.0 & 0.1 & -4.8 & -0.2 \\
\hline 0.003 & 6.5 & 0.043 & 0.0226 & 12.1 & 4.9 & 13.0 & 2.1 & 0.4 & 2.6 & 1.2 & -2.3 & -0.5 & -0.9 & -0.2 & 0.3 & 0.3 & -0.9 \\
\hline 0.003 & 6.5 & 0.067 & 0.0215 & 10.9 & 5.1 & 12.1 & 2.1 & 0.4 & -2.6 & 2.9 & -1.0 & -0.7 & -1.2 & -0.2 & -0.3 & 0.3 & -1.0 \\
\hline 0.003 & 6.5 & 0.11 & 0.0191 & 12.2 & 4.6 & 13.1 & 2.1 & 0.6 & 2.6 & 2.2 & -0.9 & -0.6 & -0.7 & 0.0 & 0.3 & 0.5 & -1.2 \\
\hline 0.003 & 6.5 & 0.17 & 0.0215 & 9.4 & 3.9 & 10.2 & 2.1 & 0.4 & -1.3 & 2.3 & -0.9 & -0.5 & -0.8 & -0.2 & 0.2 & 0.3 & 0.5 \\
\hline 0.003 & 6.5 & 0.27 & 0247 & 8.7 & 5.0 & 10.1 & 2.1 & 0.6 & -1.2 & 3.1 & -2.5 & -0.3 & -0.7 & -0.1 & -0.1 & 0.4 & 0.8 \\
\hline 0.003 & 6.5 & 0.43 & 0.0238 & 8.2 & 4.3 & 9.2 & 2.1 & 1.3 & -1.3 & 2.4 & -1.0 & 0.4 & -0.3 & 0.0 & 0.3 & 0.2 & 0.2 \\
\hline 0.003 & 8.5 & 0.043 & 0.0201 & 19.2 & 8.2 & 20.9 & 2.1 & 1.9 & 5.3 & 2.1 & 2.8 & -0.1 & -1.2 & -0.9 & 0.6 & 0.4 & 1.7 \\
\hline 0.003 & 8.5 & 0.067 & 0.0226 & 11.8 & 4.7 & 12.7 & 2.1 & -0.6 & 1.8 & -1.6 & -2.4 & -0.6 & -1.2 & -0.5 & -0.2 & 0.2 & 1.0 \\
\hline 0.003 & 8.5 & 0.11 & 0.0201 & 10.2 & 5.0 & 11.3 & 2.1 & -1.8 & -1.9 & 1.3 & -2.3 & -0.7 & -1.1 & -0.2 & -0.0 & 0.2 & -1.9 \\
\hline 0.003 & 8.5 & 0.17 & 0.0218 & 10.1 & 3.9 & 10.8 & 2.1 & 0.3 & -0.5 & 1.3 & -1.5 & -0.6 & -0.9 & -0.4 & -0.4 & 0.2 & 0.5 \\
\hline 0.003 & 8.5 & 0.27 & 0.0236 & 8.2 & 3.8 & 9.1 & 2.1 & -0.2 & -0.6 & 1.7 & -1.8 & -0.6 & -0.9 & -0.2 & 0.4 & 0.1 & -0.3 \\
\hline 0.003 & 8.5 & 0.43 & 0305 & 8.0 & 3.6 & 8.8 & 2.1 & 0.9 & -0.9 & 0.7 & -1.4 & -0.1 & -0.6 & -0.2 & -0.1 & 0.1 & -0.3 \\
\hline 0.003 & 8.5 & 0.67 & 0.0397 & 10.9 & 5.7 & 12.4 & 2.1 & 0.5 & -2.4 & 1.9 & 3.1 & 1.5 & 0.5 & -0.4 & 0.4 & 0.0 & 0.9 \\
\hline 0.003 & 12.0 & 0.067 & 0247 & 15.0 & 4.9 & 15.8 & 2.1 & 0.2 & -0.8 & 1.4 & -2.6 & -0.5 & -1.3 & -0.5 & -0.5 & 0.5 & 1.4 \\
\hline 0.003 & 12.0 & 0.11 & 0.0242 & 12.1 & 4.2 & 12.8 & 2.1 & 1.5 & 1.7 & 0.5 & 0.2 & -0.4 & -1.1 & -0.4 & 0.4 & 0.6 & 0.4 \\
\hline 0.003 & 12.0 & 0.17 & 0.0266 & 10.4 & 4.3 & 11.3 & 2.1 & -0.3 & -2.4 & 1.7 & -1.6 & -0.4 & -0.9 & -0.2 & 0.5 & 0.4 & 0.5 \\
\hline 0.003 & 12.0 & 0.27 & 0.0226 & 10.5 & 3.1 & 11.0 & 2.1 & 0.3 & -1.0 & -0.4 & -1.3 & -0.6 & -0.8 & 0.0 & -0.2 & 0.4 & 0.5 \\
\hline 0.003 & 12.0 & 0.43 & 0.0260 & 9.9 & 5.2 & 11.2 & 2.1 & 1.5 & -2.3 & 3.1 & -1.3 & -0.3 & -0.5 & 0.0 & 0.2 & 0.5 & 0.7 \\
\hline 0.003 & 12.0 & 0.67 & 0.0392 & 10.8 & 4.6 & 11.7 & 2.1 & 0.9 & 0.7 & 1.4 & 1.2 & 1.5 & 0.4 & 0.0 & -0.4 & 0.5 & 1.1 \\
\hline 0.003 & 15.0 & 0.067 & 0338 & 14.4 & 5.0 & 15.3 & 2.1 & 1.5 & 1.7 & 1.4 & 1.1 & -0.4 & -1.7 & 0.0 & 0.7 & -0.1 & 2.2 \\
\hline 0.003 & 15.0 & 0.11 & 0.0282 & 6.3 & 3.6 & 7.3 & 2.1 & 0.7 & 1.3 & 1.0 & -1.0 & -0.4 & -1.0 & -0.5 & -0.0 & 0.2 & 0.5 \\
\hline 0.003 & 15.0 & 0.17 & 0.0245 & 5.5 & 3.5 & 6.5 & 2.1 & 0.4 & -2.1 & 0.6 & -0.6 & -0.6 & -1.0 & 0.0 & 0.2 & 0.2 & 0.2 \\
\hline 0.003 & 15.0 & 0.27 & 0.0273 & 4.9 & 3.5 & 6.0 & 2.1 & 0.3 & -1.0 & 1.1 & -1.6 & -0.5 & -0.8 & -0.2 & 0.2 & -0.0 & 0.2 \\
\hline 0.003 & 15.0 & 0.43 & 0.0331 & 4.9 & 3.6 & 6.1 & 2.1 & 0.5 & -1.2 & 1.9 & 0.7 & -0.4 & -0.5 & -0.2 & -0.1 & 0.1 & 0.4 \\
\hline 0.003 & 15.0 & 0.67 & 0.0381 & 5.8 & 4.0 & 7.1 & 2.1 & 0.5 & -1.5 & 1.6 & 1.7 & 1.0 & 0.5 & -0.1 & 0.7 & 0.1 & 0.6 \\
\hline 0.003 & 20.0 & 0.11 & 0.0276 & 9.0 & 4.1 & 9.9 & 2.1 & 0.2 & 2.5 & 0.4 & -1.5 & -0.4 & -1.2 & -0.4 & 0.2 & 0.4 & 1.3 \\
\hline 0.003 & 20.0 & 0.17 & 0.0281 & 6.3 & 3.5 & 7.2 & 2.1 & 0.5 & -1.5 & 1.0 & -0.7 & -0.2 & -1.1 & -0.9 & 0.2 & 0.4 & -0.3 \\
\hline 0.003 & 20.0 & 0.27 & 0.0321 & 5.9 & 3.6 & 6.9 & 2.1 & -0.1 & -1.4 & 0.8 & -1.8 & -0.4 & -0.9 & -0.1 & -0.1 & 0.3 & -0.3 \\
\hline 0.003 & 20.0 & 0.43 & 0.0324 & 5.2 & 3.5 & 6.3 & 2.1 & 0.4 & -1.7 & 1.2 & -0.6 & -0.2 & -0.4 & -0.2 & -0.1 & 0.3 & 0.4 \\
\hline 0.003 & 20.0 & 0.67 & 0.0411 & 6.3 & 3.6 & 7.3 & 2.1 & 0.7 & -1.6 & 1.1 & 1.4 & 0.8 & 0.5 & -0.1 & 0.1 & 0.3 & 0.3 \\
\hline 0.003 & 25.0 & 0.11 & 0.0343 & 20.2 & 6.3 & 21.2 & 2.1 & -1.3 & -4.2 & 1.4 & -2.1 & -0.3 & -1.6 & 0.0 & -0.0 & 0.1 & 2.0 \\
\hline 0.003 & 25.0 & 0.17 & 0.0335 & 7.2 & 3.1 & 7.8 & 2.1 & -0.0 & 0.8 & 0.4 & 0.9 & -0.5 & -1.0 & 0.0 & 0.5 & 0.1 & 1.1 \\
\hline
\end{tabular}




\begin{tabular}{|c|c|c|c|c|c|c|c|c|c|c|c|c|c|c|c|c|c|}
\hline$x_{\mathbb{P}}$ & $\begin{array}{c}Q^{2} \\
{\left[\mathrm{GeV}^{2}\right]}\end{array}$ & $\beta$ & $x_{\mathbb{P}} \sigma_{r}^{D(3)}$ & $\begin{array}{c}\delta_{\text {stat }} \\
{[\%]}\end{array}$ & $\begin{array}{l}\delta_{\text {sys }} \\
{[\%]}\end{array}$ & $\begin{array}{l}\delta_{t o t} \\
{[\%]}\end{array}$ & $\begin{array}{c}\delta_{\text {unc }} \\
{[\%]}\end{array}$ & $\begin{array}{l}\delta_{l a r} \\
{[\%]}\end{array}$ & $\begin{array}{l}\delta_{\text {ele }} \\
{[\%]}\end{array}$ & $\begin{array}{c}\delta_{\theta} \\
{[\%]}\end{array}$ & $\begin{array}{c}\delta_{\text {noise }} \\
{[\%]}\end{array}$ & $\begin{array}{l}\delta_{x_{\mathbb{P}}} \\
{[\%]}\end{array}$ & $\begin{array}{c}\delta_{\beta} \\
{[\%]}\end{array}$ & $\begin{array}{c}\delta_{b g} \\
{[\%]}\end{array}$ & $\begin{array}{c}\delta_{\text {Plug }} \\
{[\%]}\end{array}$ & $\begin{array}{l}\delta_{Q^{2}} \\
{[\%]}\end{array}$ & $\begin{array}{l}\delta_{s p a} \\
{[\%]} \\
\end{array}$ \\
\hline 0.003 & 25.0 & 0.27 & 0.0338 & 6.8 & 3.7 & 7.7 & 2.1 & 0.3 & -1.9 & 1.1 & -1.3 & -0.3 & -0.9 & -0.2 & -0.3 & -0.0 & -0.4 \\
\hline 0.003 & 25.0 & 0.43 & 0.0354 & 5.8 & 3.7 & 6.8 & 2.1 & 0.8 & -2.0 & 0.8 & 1.1 & -0.3 & -0.4 & -0.2 & 0.4 & -0.0 & 0.3 \\
\hline 0.003 & 25.0 & 0.67 & 0.0349 & 6.7 & 3.9 & 7.7 & 2.1 & 0.6 & -1.8 & 1.2 & 1.4 & 0.9 & 0.5 & 0.0 & -0.2 & 0.0 & 0.4 \\
\hline 0.003 & 35.0 & 0.17 & 0.0423 & 13.2 & 4.8 & 14.1 & 2.1 & 0.6 & 1.1 & 0.8 & -0.9 & -0.5 & -1.5 & -0.7 & 0.2 & 0.7 & 3.1 \\
\hline 0.003 & 35.0 & 0.27 & 0.0387 & 6.9 & 3.0 & 7.5 & 2.1 & 0.6 & -1.1 & 0.2 & -0.2 & -0.4 & -1.0 & -0.4 & -0.3 & 0.4 & 0.7 \\
\hline 0.003 & 35.0 & 0.43 & 0.0353 & 6.7 & 3.8 & 7.6 & 2.1 & 0.6 & -2.3 & 1.0 & -0.6 & -0.2 & -0.5 & -0.4 & 0.3 & 0.3 & 0.1 \\
\hline 0.003 & 35.0 & 0.67 & 0.0371 & 7.7 & 3.7 & 8.5 & 2.1 & 0.5 & -1.9 & 0.9 & 0.9 & 0.9 & 0.5 & -0.2 & 0.3 & 0.3 & 0.8 \\
\hline 0.003 & 45.0 & 0.27 & 0.0326 & 19.7 & 3.9 & 20.1 & 2.1 & -0.3 & -0.8 & 0.8 & -1.3 & -0.4 & -1.2 & 0.0 & -0.0 & 0.3 & 1.8 \\
\hline 0.003 & 45.0 & 0.43 & 0.0365 & 9.1 & 3.9 & 9.9 & 2.1 & 0.7 & -1.6 & 1.3 & 0.4 & 0.2 & -0.4 & -0.6 & 0.1 & 0.2 & 0.9 \\
\hline 0.003 & 45.0 & 0.67 & 0.0383 & 9.5 & 4.7 & 10.6 & 2.1 & 0.6 & -3.4 & 0.9 & 1.2 & 0.7 & 0.5 & -0.3 & 0.2 & 0.0 & 0.7 \\
\hline 0.003 & 60.0 & 0.43 & 0.0406 & 14.5 & 5.6 & 15.5 & 2.1 & 1.2 & -3.8 & 1.0 & 1.3 & 0.1 & -0.7 & 0.0 & -0.6 & 0.5 & 1.6 \\
\hline 0.003 & 60.0 & 0.67 & 0.0429 & 10.6 & 4.0 & 11.3 & 2.1 & 0.4 & -2.2 & 0.6 & 1.4 & 0.6 & 0.5 & 0.0 & 0.1 & 0.1 & 0.8 \\
\hline 0.003 & 90.0 & 0.67 & 0.0366 & 40.9 & 10.0 & 42.1 & 2.1 & 1.0 & 3.8 & 4.8 & 4.2 & -0.9 & 0.7 & 0.0 & 0.0 & -0.4 & 1.3 \\
\hline 0.01 & 3.5 & 0.005 & 0.0237 & 15.3 & 8.8 & 17.6 & 3.6 & 1.1 & 1.7 & 3.4 & -0.6 & -0.9 & -2.4 & -1.2 & 0.2 & 0.6 & -3.2 \\
\hline 0.01 & 3.5 & 0.008 & 0.0233 & 8.7 & 6.4 & 10.8 & 3.6 & 0.9 & 1.8 & 0.8 & 0.5 & -0.1 & -2.0 & -2.7 & 0.5 & 0.6 & 1.8 \\
\hline 0.01 & 3.5 & 0.013 & 0.0213 & 8.7 & 6.5 & 10.8 & 3.6 & 0.7 & 3.3 & 0.9 & 0.2 & -0.5 & -1.6 & -2.3 & 0.5 & 0.7 & -2.1 \\
\hline 0.01 & 3.5 & 0.02 & 0.0173 & 7.2 & 5.3 & 9.0 & 3.6 & 1.2 & 1.8 & 0.7 & 1.2 & -0.2 & -1.2 & -1.7 & -0.6 & 0.3 & 1.4 \\
\hline 0.01 & 3.5 & 0.032 & 0.0172 & 8.4 & 6.0 & 10.3 & 3.6 & 2.5 & 0.7 & 2.4 & 1.0 & -0.6 & -0.9 & -1.3 & 0.2 & 0.1 & -0.5 \\
\hline 0.01 & 3.5 & 0.05 & 0.0153 & 8.7 & 6.2 & 10.7 & 3.6 & 0.8 & -2.2 & 1.3 & 0.4 & 0.1 & -0.8 & -2.9 & 0.9 & -0.2 & 0.1 \\
\hline 0.01 & 3.5 & 0.08 & 0.0167 & 9.9 & 7.2 & 12.3 & 3.6 & 2.4 & 0.5 & 2.6 & 1.6 & 0.2 & -1.3 & -2.4 & 0.3 & -0.6 & -1.1 \\
\hline 0.01 & 5.0 & 0.008 & 0.0183 & 13.1 & 8.5 & 15.6 & 3.6 & 2.4 & 3.8 & 2.3 & -2.3 & -0.1 & -1.7 & -1.6 & 0.9 & 2.0 & 0.3 \\
\hline 0.01 & 5.0 & 0.013 & 0.0234 & 9.4 & 5.5 & 10.9 & 3.6 & -1.0 & 2.6 & 1.4 & 0.6 & -0.1 & -1.1 & -1.6 & 0.4 & 1.3 & -0.3 \\
\hline 0.01 & 5.0 & 0.02 & 0.0226 & 8.6 & 5.5 & 10.2 & 3.6 & 1.7 & -1.3 & 1.9 & -0.4 & -0.1 & -1.2 & -2.1 & 0.4 & 0.3 & 0.7 \\
\hline 0.01 & 5.0 & 0.032 & 0.0208 & 9.9 & 5.6 & 11.4 & 3.6 & 1.4 & 1.2 & 1.2 & 1.9 & -0.1 & -0.8 & -1.9 & 1.3 & 0.5 & -1.6 \\
\hline 0.01 & 5.0 & 0.05 & 0.0235 & 9.5 & 6.3 & 11.4 & 3.6 & -0.8 & -2.7 & 2.2 & -0.7 & -0.3 & -0.9 & -2.4 & 0.4 & 0.2 & 0.5 \\
\hline 0.01 & 5.0 & 0.08 & 0.0245 & 12.5 & 7.8 & 14.7 & 3.6 & 0.8 & -1.7 & 2.1 & -1.2 & -1.3 & -1.1 & -2.2 & 0.9 & -1.1 & -0.5 \\
\hline 0.01 & 6.5 & 0.013 & 0.0209 & 11.7 & 5.5 & 12.9 & 3.6 & 1.7 & 2.1 & -0.6 & 0.5 & -0.3 & -1.5 & -1.8 & 0.5 & 0.3 & 0.3 \\
\hline 0.01 & 6.5 & 0.02 & 0.0232 & 9.5 & 5.1 & 10.8 & 3.6 & 0.9 & -1.0 & 0.8 & 1.2 & -0.1 & -1.1 & -1.5 & 0.5 & 0.3 & -0.2 \\
\hline 0.01 & 6.5 & 0.032 & 0.0253 & 9.1 & 5.8 & 10.8 & 3.6 & 1.1 & -2.2 & 1.3 & -2.2 & -0.2 & -1.0 & -1.5 & 0.8 & 0.3 & -1.1 \\
\hline 0.01 & 6.5 & 0.05 & 0.0272 & 12.5 & 6.7 & 14.2 & 3.6 & 0.3 & 0.5 & 0.5 & -1.0 & -1.0 & -1.5 & -1.7 & 0.9 & -1.2 & 0.6 \\
\hline 0.01 & 6.5 & 0.08 & 0.0225 & 8.6 & 6.1 & 10.6 & 3.6 & -1.7 & -1.0 & 2.5 & 1.1 & 0.2 & -0.8 & -2.7 & -0.1 & 0.3 & -0.2 \\
\hline 0.01 & 6.5 & 0.13 & 0.0203 & 11.2 & 6.1 & 12.8 & 3.6 & 1.1 & 1.6 & -2.0 & 1.0 & 0.3 & -1.0 & -1.8 & 0.6 & 0.1 & 1.2 \\
\hline 0.01 & 8.5 & 0.013 & 0.0293 & 16.6 & 7.8 & 18.3 & 3.6 & 1.0 & 4.4 & -2.7 & -1.1 & -0.4 & -1.6 & -1.0 & 1.8 & 0.4 & -1.1 \\
\hline 0.01 & 8.5 & 0.02 & 0.0244 & 10.2 & 5.9 & 11.8 & 3.6 & -0.9 & 0.4 & 2.8 & -1.3 & -0.4 & -1.6 & -1.7 & 0.5 & 0.1 & 0.9 \\
\hline 0.01 & 8.5 & 0.032 & 0.0300 & 8.7 & 5.3 & 10.2 & 3.6 & 0.7 & 1.3 & 1.3 & 1.7 & 0.2 & -1.1 & -2.3 & 0.8 & 0.0 & 0.5 \\
\hline 0.01 & 8.5 & 0.05 & 0.0227 & 9.0 & 5.3 & 10.5 & 3.6 & 0.8 & -1.4 & 2.4 & 1.4 & -0.2 & -0.7 & -0.9 & 0.6 & 0.2 & 0.8 \\
\hline
\end{tabular}




\begin{tabular}{|c|c|c|c|c|c|c|c|c|c|c|c|c|c|c|c|c|c|c|}
\hline & $x_{\mathbb{P}}$ & $\begin{array}{c}Q^{2} \\
{\left[\mathrm{GeV}^{2}\right]}\end{array}$ & $\beta$ & $x_{\mathbb{P}} \sigma_{r}^{D(3)}$ & $\begin{array}{c}\delta_{\text {stat }} \\
{[\%]}\end{array}$ & $\begin{array}{c}\delta_{\text {sys }} \\
{[\%]}\end{array}$ & $\begin{array}{l}\delta_{t o t} \\
{[\%]}\end{array}$ & $\begin{array}{c}\delta_{\text {unc }} \\
{[\%]}\end{array}$ & $\begin{array}{l}\delta_{l a r} \\
{[\%]}\end{array}$ & $\begin{array}{l}\delta_{\text {ele }} \\
{[\%]}\end{array}$ & $\begin{array}{c}\delta_{\theta} \\
{[\%]}\end{array}$ & $\begin{array}{c}\delta_{\text {noise }} \\
{[\%]}\end{array}$ & $\begin{array}{l}\delta_{x_{I P}} \\
{[\%]}\end{array}$ & $\begin{array}{c}\delta_{\beta} \\
{[\%]}\end{array}$ & $\begin{array}{c}\delta_{b g} \\
{[\%]}\end{array}$ & $\begin{array}{c}\delta_{\text {Plug }} \\
{[\%]}\end{array}$ & $\begin{array}{l}\delta_{Q^{2}} \\
{[\%]}\end{array}$ & $\begin{array}{l}\delta_{\text {spa }} \\
{[\%]} \\
\end{array}$ \\
\hline & 0.01 & 8.5 & 0.08 & 0.0182 & 9.4 & 5.2 & 10.8 & 3.6 & 0.9 & 1.6 & 0.9 & -1.5 & -0.2 & -1.0 & -2.0 & 0.7 & -0.1 & 0.3 \\
\hline & 0.01 & 8.5 & 0.13 & 0.0200 & 9.1 & 6.7 & 11.3 & 3.6 & -0.2 & -1.9 & 1.6 & -1.0 & 0.6 & -0.9 & -2.9 & 0.8 & 0.3 & -0.5 \\
\hline & 0.01 & 8.5 & 0.2 & 0.0220 & 10.2 & 6.4 & 12.0 & 3.6 & 1.5 & -1.3 & -1.2 & -2.1 & -0.2 & -1.0 & -0.7 & 0.3 & -0.4 & -0.6 \\
\hline & 0.01 & 12.0 & 0.02 & 0.0370 & 12.7 & 5.4 & 13.8 & 3.6 & 0.5 & 1.8 & -1.1 & -0.9 & -0.2 & -1.6 & -1.8 & 0.1 & 0.4 & 0.4 \\
\hline & 0.01 & 12.0 & 0.032 & 0.0340 & 10.3 & 5.1 & 11.4 & 3.6 & 1.0 & -1.4 & -0.5 & 1.2 & -0.3 & -1.4 & -1.3 & 0.3 & 0.5 & -0.8 \\
\hline & 0.01 & 12.0 & 0.05 & 0.0345 & 9.6 & 5.2 & 10.9 & 3.6 & 0.6 & -1.2 & 1.6 & -1.0 & 0.1 & -1.1 & -2.3 & 0.6 & 0.5 & 0.4 \\
\hline & 0.01 & 12.0 & 0.08 & 0.0259 & 10.1 & 5.6 & 11.6 & 3.6 & 0.7 & 1.4 & 1.0 & -1.9 & -0.2 & -1.1 & -1.8 & 0.4 & 0.5 & -1.7 \\
\hline & 0.01 & 12.0 & 0.13 & 0.0217 & 10.2 & 5.2 & 11.4 & 3.6 & 0.9 & -1.9 & 0.4 & 1.7 & -0.1 & -0.9 & -1.6 & 0.2 & 0.6 & 0.2 \\
\hline & 0.01 & 12.0 & 0.2 & 0.0258 & 11.9 & 5.2 & 13.0 & 3.6 & -1.2 & -0.6 & 2.5 & -0.8 & 0.4 & -1.1 & 0.0 & -0.5 & 0.8 & 0.7 \\
\hline & 0.01 & 12.0 & 0.32 & 0.0240 & 14.0 & 5.3 & 14.9 & 3.6 & -0.5 & -1.3 & 1.5 & -1.1 & 0.8 & -0.0 & -0.4 & -0.4 & 0.4 & -2.3 \\
\hline & 0.01 & 15.0 & 0.02 & 0.0329 & 14.2 & 6.2 & 15.4 & 3.6 & 1.3 & -2.0 & 0.4 & -1.9 & -0.4 & -2.0 & -0.7 & -1.8 & -0.2 & 1.5 \\
\hline & 0.01 & 15.0 & 0.032 & 0.0306 & 6.0 & 5.3 & 8.0 & 3.6 & 0.3 & 1.7 & 1.0 & -0.5 & -0.1 & -1.3 & -1.8 & 0.2 & 0.2 & 1.0 \\
\hline & 0.01 & 15.0 & 0.05 & 0.0291 & 5.3 & 4.8 & 7.1 & 3.6 & -0.5 & -1.3 & 0.7 & -0.9 & -0.2 & -1.4 & -1.7 & 0.5 & 0.1 & -0.7 \\
\hline & 0.01 & 15.0 & 0.08 & 0.0232 & 6.8 & 4.9 & 8.4 & 3.6 & 0.1 & 0.6 & 0.7 & -0.6 & 0.1 & -1.2 & -2.6 & 0.7 & 0.1 & 0.3 \\
\hline & 0.01 & 15.0 & 0.13 & 0.0230 & 5.1 & 5.0 & 7.2 & 3.6 & 0.5 & -0.4 & 0.9 & -1.1 & -0.1 & -1.1 & -2.7 & 0.3 & 0.1 & 0.3 \\
\hline & 0.01 & 15.0 & 0.2 & 0.0224 & 5.0 & 4.9 & 7.0 & 3.6 & 0.7 & 0.7 & 0.6 & -1.0 & 0.5 & -1.2 & -2.5 & 0.4 & 0.2 & 0.3 \\
\hline$r$ & 0.01 & 15.0 & 0.32 & 0.0229 & 5.2 & 4.5 & 6.9 & 3.6 & 0.6 & -1.4 & -0.6 & -0.2 & 0.4 & -0.8 & -1.0 & 0.2 & 0.1 & 0.2 \\
\hline & 0.01 & 20.0 & 0.032 & 0.0337 & 8.8 & 5.7 & 10.5 & 3.6 & 1.2 & -2.1 & 1.3 & -0.9 & -0.1 & -1.5 & -2.1 & 0.9 & 0.4 & 1.3 \\
\hline & 0.01 & 20.0 & 0.05 & 0.0287 & 6.8 & 5.3 & 8.6 & 3.6 & 0.7 & -1.1 & 1.1 & -0.8 & 0.2 & -1.4 & -2.8 & 0.5 & 0.6 & -0.3 \\
\hline & 0.01 & 20.0 & 0.08 & 0.0274 & 5.8 & 4.7 & 7.4 & 3.6 & 0.3 & -1.2 & 1.1 & 0.2 & -0.1 & -1.1 & -1.5 & -0.6 & 0.4 & -0.3 \\
\hline & 0.01 & 20.0 & 0.13 & 0.0270 & 5.6 & 4.9 & 7.4 & 3.6 & 0.8 & -0.6 & 0.7 & -0.7 & -0.1 & -1.0 & -2.5 & 0.6 & 0.4 & -0.2 \\
\hline & 0.01 & 20.0 & 0.2 & 0.0241 & 9.3 & 5.0 & 10.5 & 3.6 & -0.4 & -1.2 & 1.5 & -0.6 & -0.1 & -1.1 & -2.0 & 0.3 & 0.4 & -0.7 \\
\hline & 0.01 & 20.0 & 0.32 & 0.0259 & 5.4 & 4.9 & 7.3 & 3.6 & 0.7 & 1.0 & 0.8 & 1.2 & 0.5 & -1.0 & -2.1 & 0.3 & 0.4 & 0.4 \\
\hline & 0.01 & 20.0 & 0.5 & 0.0294 & 6.9 & 5.4 & 8.8 & 3.6 & 0.3 & -2.2 & 1.0 & -0.9 & 0.8 & -0.2 & -2.1 & -0.5 & 0.3 & 0.8 \\
\hline & 0.01 & 25.0 & 0.032 & 0.0289 & 20.1 & 6.0 & 20.9 & 3.6 & -1.1 & -1.8 & -0.7 & 1.1 & -0.2 & -2.0 & -1.8 & 0.5 & 0.2 & -1.5 \\
\hline & 0.01 & 25.0 & 0.05 & 0.0335 & 7.5 & 5.2 & 9.1 & 3.6 & 1.1 & 1.4 & 0.6 & -0.7 & 0.1 & -1.4 & -2.6 & 0.4 & 0.3 & 0.6 \\
\hline & 0.01 & 25.0 & 0.08 & 0.0315 & 5.9 & 5.2 & 7.9 & 3.6 & 0.5 & -1.2 & 0.4 & -0.2 & -0.1 & -1.2 & -2.1 & 0.3 & 0.2 & 0.3 \\
\hline & 0.01 & 25.0 & 0.13 & 0.0270 & 6.0 & 4.4 & 7.4 & 3.6 & 0.4 & -0.8 & 0.7 & 0.7 & -0.2 & -1.0 & -1.5 & 0.5 & 0.1 & -0.5 \\
\hline & 0.01 & 25.0 & 0.2 & 0.0253 & 5.7 & 5.0 & 7.6 & 3.6 & 0.5 & 1.1 & 1.3 & -0.3 & -0.1 & -1.2 & -2.3 & 0.4 & 0.2 & 0.9 \\
\hline & 0.01 & 25.0 & 0.32 & 0.0242 & 5.8 & 5.0 & 7.7 & 3.6 & 1.2 & -0.9 & 1.2 & 0.4 & 0.4 & -1.0 & -2.1 & 0.7 & 0.2 & -0.1 \\
\hline & 0.01 & 25.0 & 0.5 & 0.0289 & 6.2 & 5.2 & 8.1 & 3.6 & 1.1 & -2.0 & 1.1 & 1.5 & 0.9 & -0.2 & -1.6 & 0.4 & 0.2 & 1.0 \\
\hline & 0.01 & 35.0 & 0.05 & 0.0299 & 14.1 & 6.1 & 15.4 & 3.6 & 0.9 & 1.3 & 1.6 & -0.5 & 0.1 & -1.7 & -2.7 & 0.3 & 0.8 & 1.5 \\
\hline & 0.01 & 35.0 & 0.08 & 0.0324 & 7.1 & 5.1 & 8.7 & 3.6 & 0.5 & -3.0 & 0.3 & 0.4 & -0.2 & -1.0 & -0.9 & 0.6 & 0.5 & 0.2 \\
\hline & 0.01 & 35.0 & 0.13 & 0.0294 & 7.0 & 4.7 & 8.4 & 3.6 & 0.4 & -1.1 & 0.4 & 0.4 & -0.1 & -1.1 & -2.3 & -0.3 & 0.4 & 0.3 \\
\hline & 0.01 & 35.0 & 0.2 & 0.0302 & 6.7 & 4.8 & 8.2 & 3.6 & 0.1 & -0.9 & 1.2 & 0.4 & -0.1 & -1.0 & -1.8 & 0.2 & 0.4 & -0.6 \\
\hline & 0.01 & 35.0 & 0.32 & 0.0232 & 6.7 & 6.6 & 9.4 & 3.6 & 0.5 & -0.5 & 0.8 & 1.2 & 0.8 & -1.1 & -4.9 & 0.6 & 0.7 & 0.7 \\
\hline
\end{tabular}




\begin{tabular}{|c|c|c|c|c|c|c|c|c|c|c|c|c|c|c|c|c|c|}
\hline$x_{\mathbb{P}}$ & $\begin{array}{c}Q^{2} \\
{\left[\mathrm{GeV}^{2}\right]}\end{array}$ & $\beta$ & $x_{\mathbb{P}} \sigma_{r}^{D(3)}$ & $\begin{array}{c}\delta_{\text {stat }} \\
{[\%]} \\
\end{array}$ & $\begin{array}{l}\delta_{\text {sys }} \\
{[\%]}\end{array}$ & $\begin{array}{l}\delta_{t o t} \\
{[\%]}\end{array}$ & $\begin{array}{c}\delta_{u n c} \\
{[\%]} \\
\end{array}$ & $\begin{array}{l}\delta_{l a r} \\
{[\%]}\end{array}$ & $\begin{array}{l}\delta_{\text {ele }} \\
{[\%]}\end{array}$ & $\begin{array}{c}\delta_{\theta} \\
{[\%]}\end{array}$ & $\begin{array}{c}\delta_{\text {noise }} \\
{[\%]}\end{array}$ & $\begin{array}{l}\delta_{x \mathbb{P}} \\
{[\%]}\end{array}$ & $\begin{array}{c}\delta_{\beta} \\
{[\%]}\end{array}$ & $\begin{array}{c}\delta_{b g} \\
{[\%]}\end{array}$ & $\begin{array}{c}\delta_{\text {Plug }} \\
{[\%]}\end{array}$ & $\begin{array}{l}\delta_{Q^{2}} \\
{[\%]}\end{array}$ & $\begin{array}{l}\delta_{\text {spa }} \\
{[\%]} \\
\end{array}$ \\
\hline 0.01 & 35.0 & 0.5 & 0.0305 & 6.6 & 4.8 & 8.2 & 3.6 & 0.6 & -1.5 & 0.9 & 0.8 & 0.8 & -0.3 & -1.2 & -0.2 & 0.6 & $\overline{0.3}$ \\
\hline 0.01 & 35.0 & 0.8 & 0.0362 & 9.2 & 7.0 & 11.6 & 3.6 & 1.0 & -1.1 & 1.7 & 2.4 & 2.4 & 1.2 & -1.9 & -1.3 & 0.6 & 1.6 \\
\hline 0.01 & 45.0 & 0.08 & 0.0329 & 13.5 & 5.3 & 14.5 & 3.6 & 1.6 & 0.8 & 1.3 & -0.7 & -0.1 & -1.4 & -1.3 & -0.6 & 0.4 & 1.9 \\
\hline 0.01 & 45.0 & 0.13 & 0.0377 & 7.7 & 6.0 & 9.7 & 3.6 & -0.8 & -2.5 & 0.9 & -1.3 & 0.1 & -1.0 & -2.4 & -0.4 & 0.3 & -1.5 \\
\hline 0.01 & 45.0 & 0.2 & 0.0264 & 8.2 & 4.7 & 9.5 & 3.6 & 0.9 & -1.5 & 0.6 & -0.5 & -0.2 & -1.0 & -1.3 & -0.9 & 0.2 & -0.7 \\
\hline 0.01 & 45.0 & 0.32 & 0.0237 & 10.6 & 6.1 & 12.2 & 3.6 & 1.5 & -1.6 & 0.9 & 0.4 & 0.6 & -1.0 & -3.6 & 0.1 & 0.5 & -0.4 \\
\hline 0.01 & 45.0 & 0.5 & 0.0373 & 8.3 & 4.9 & 9.7 & 3.6 & -0.7 & -1.1 & -0.3 & 0.9 & 0.6 & -0.4 & -1.5 & -0.2 & 0.3 & -0.8 \\
\hline 0.01 & 45.0 & 0.8 & 0.0245 & 11.2 & 5.9 & 12.7 & 3.6 & 1.2 & -1.4 & 1.1 & 2.3 & 1.8 & 1.0 & -0.9 & 0.4 & 0.3 & 0.4 \\
\hline 0.01 & 60.0 & 0.13 & 0.0379 & 13.3 & 6.5 & 14.8 & 3.6 & 1.5 & -2.6 & 0.9 & 2.9 & -0.1 & -1.2 & -1.5 & 0.9 & 0.5 & 0.8 \\
\hline 0.01 & 60.0 & 0.2 & 0.0318 & 8.9 & 5.4 & 10.4 & 3.6 & 1.1 & -1.3 & 1.2 & -0.5 & 0.1 & -1.2 & -2.7 & -0.2 & 0.3 & 0.7 \\
\hline 0.01 & 60.0 & 0.32 & 0.0319 & 8.0 & 4.9 & 9.4 & 3.6 & 0.8 & -2.1 & 0.5 & 0.6 & 0.1 & -0.8 & -1.6 & 0.5 & 0.2 & 0.1 \\
\hline 0.01 & 60.0 & 0.5 & 0.0335 & 10.8 & 6.9 & 12.8 & 3.6 & 0.8 & -1.5 & 0.9 & 0.5 & 1.6 & -0.2 & -5.0 & 0.2 & 0.7 & 0.8 \\
\hline 0.01 & 60.0 & 0.8 & 0.0277 & 12.3 & 7.0 & 14.2 & 3.6 & 0.9 & -3.5 & 0.5 & 1.5 & 1.7 & 1.0 & -2.9 & 0.3 & 0.3 & 1.5 \\
\hline 0.01 & 90.0 & 0.2 & 0.0259 & 35.3 & 8.9 & 36.4 & 3.6 & 0.6 & -5.4 & 3.8 & 1.2 & -0.6 & -1.6 & 0.0 & -1.2 & 0.8 & -2.3 \\
\hline 0.01 & 90.0 & 0.32 & 0.0292 & 14.4 & 5.5 & 15.5 & 3.6 & 1.1 & 2.3 & 1.7 & 1.5 & -0.0 & -0.7 & -0.6 & -0.5 & 0.6 & 0.8 \\
\hline 0.01 & 90.0 & 0.5 & 0.0326 & 12.9 & 7.3 & 14.8 & 3.6 & 1.2 & -2.7 & 1.7 & 1.8 & 0.6 & -0.2 & -2.0 & 0.8 & 0.6 & 1.6 \\
\hline 0.01 & 90.0 & 0.8 & 0.0240 & 16.5 & 6.2 & 17.6 & 3.6 & 0.9 & -0.3 & 0.3 & 1.7 & 2.1 & 0.8 & -2.4 & -0.9 & 0.9 & 1.5 \\
\hline 0.03 & 3.5 & 0.0017 & 0.0188 & 25.3 & 17.0 & 30.5 & 11.2 & -6.2 & 4.6 & -2.1 & -2.9 & 0.8 & -2.3 & -4.2 & -2.4 & 0.7 & 0.9 \\
\hline 0.03 & 3.5 & 0.0027 & 0.0263 & 12.7 & 16.3 & 20.7 & 11.2 & -2.1 & -1.3 & 4.4 & -2.9 & 0.7 & -2.8 & -7.1 & 4.8 & -0.7 & 1.3 \\
\hline 0.03 & 3.5 & 0.0043 & 0.0254 & 10.8 & 17.3 & 20.3 & 11.2 & -1.3 & 7.5 & 2.5 & -3.4 & 0.9 & -2.2 & -7.0 & 2.7 & -0.4 & 1.2 \\
\hline 0.03 & 3.5 & 0.0067 & 0.0214 & 11.6 & 14.5 & 18.6 & 11.2 & -2.8 & -2.2 & -1.1 & -3.3 & 1.2 & -2.2 & -6.3 & 2.2 & 0.8 & 1.1 \\
\hline 0.03 & 3.5 & 0.011 & 0.0190 & 12.5 & 14.6 & 19.2 & 11.2 & -2.3 & -1.9 & 3.0 & -2.8 & 1.1 & -1.1 & -6.0 & -2.9 & -1.2 & -1.2 \\
\hline 0.03 & 5.0 & 0.0027 & 0.0366 & 16.2 & 26.1 & 30.7 & 11.2 & -4.6 & -17.1 & -2.4 & -3.4 & 1.2 & -3.2 & -8.2 & 4.3 & -0.2 & -8.6 \\
\hline 0.03 & 5.0 & 0.0043 & 0.0325 & 12.9 & 15.9 & 20.5 & 11.2 & -0.7 & 8.5 & -2.4 & -2.6 & 0.6 & -1.2 & -3.9 & 1.7 & 0.4 & -1.2 \\
\hline 0.03 & 5.0 & 0.0067 & 0.0302 & 11.0 & 15.2 & 18.7 & 11.2 & 1.9 & -2.7 & 0.5 & -2.4 & 1.2 & -2.7 & -7.7 & 3.2 & 0.5 & 0.4 \\
\hline 0.03 & 5.0 & 0.011 & 0.0233 & 13.3 & 14.4 & 19.6 & 11.2 & -1.8 & 4.3 & -0.5 & -1.5 & 1.0 & -1.3 & -6.0 & 2.5 & -1.0 & -2.7 \\
\hline 0.03 & 5.0 & 0.017 & 0.0225 & 11.0 & 15.6 & 19.1 & 11.2 & 0.2 & -4.6 & 1.8 & -2.3 & 2.1 & -1.1 & -6.6 & 3.8 & 0.7 & 2.6 \\
\hline 0.03 & 6.5 & 0.0027 & 0.0366 & 33.5 & 17.5 & 37.8 & 11.2 & 0.9 & -8.9 & -1.4 & 2.7 & -0.1 & -3.4 & -3.4 & 0.1 & -0.4 & -3.5 \\
\hline 0.03 & 6.5 & 0.0043 & 0.0211 & 14.6 & 16.0 & 21.7 & 11.2 & 3.1 & 2.1 & 3.6 & 2.5 & 1.5 & -1.6 & -6.2 & 5.5 & 0.1 & 3.3 \\
\hline 0.03 & 6.5 & 0.0067 & 0.0261 & 12.0 & 15.3 & 19.4 & 11.2 & -1.7 & -2.0 & 1.5 & -3.4 & 1.3 & -2.6 & -7.5 & 1.6 & 0.3 & -1.9 \\
\hline 0.03 & 6.5 & 0.011 & 0.0263 & 12.0 & 14.4 & 18.7 & 11.2 & -2.3 & 0.4 & 2.4 & -2.1 & 1.0 & -0.7 & -3.9 & 5.2 & 0.2 & 2.1 \\
\hline 0.03 & 6.5 & 0.017 & 0.0266 & 11.6 & 13.8 & 18.0 & 11.2 & -0.8 & 0.7 & -2.0 & -1.3 & 1.3 & -0.8 & -5.7 & -1.6 & -0.1 & 0.2 \\
\hline 0.03 & 6.5 & 0.027 & 0.0262 & 10.3 & 15.0 & 18.2 & 11.2 & -1.6 & 0.5 & 1.2 & -2.1 & 1.5 & -1.7 & -7.4 & 2.6 & 0.5 & -0.3 \\
\hline 0.03 & 6.5 & 0.043 & 0.0225 & 12.1 & 14.7 & 19.1 & 11.2 & 1.3 & 2.3 & 3.8 & 2.6 & 1.4 & -0.8 & -6.9 & 0.5 & 0.0 & 1.0 \\
\hline 0.03 & 8.5 & 0.0043 & 0.0331 & 18.8 & 16.9 & 25.2 & 11.2 & -5.5 & -2.0 & 2.4 & -3.4 & 0.7 & -1.8 & -4.2 & 1.8 & 0.4 & -6.0 \\
\hline 0.03 & 8.5 & 0.0067 & 0.0250 & 13.1 & 14.7 & 19.7 & 11.2 & 0.8 & 2.8 & -0.1 & -2.6 & 1.4 & -0.9 & -4.8 & 1.8 & 0.4 & 2.8 \\
\hline
\end{tabular}




\begin{tabular}{|c|c|c|c|c|c|c|c|c|c|c|c|c|c|c|c|c|c|}
\hline$x_{\mathbb{P}}$ & $\begin{array}{c}Q^{2} \\
{\left[\mathrm{GeV}^{2}\right]}\end{array}$ & $\beta$ & $x_{\mathbb{P}} \sigma_{r}^{D(3)}$ & $\begin{array}{c}\delta_{\text {stat }} \\
{[\%]}\end{array}$ & $\begin{array}{l}\delta_{\text {sys }} \\
{[\%]}\end{array}$ & $\begin{array}{l}\delta_{t o t} \\
{[\%]}\end{array}$ & $\begin{array}{c}\delta_{\text {unc }} \\
{[\%]}\end{array}$ & $\begin{array}{l}\delta_{\text {lar }} \\
{[\%]}\end{array}$ & $\begin{array}{l}\delta_{\text {ele }} \\
{[\%]}\end{array}$ & $\begin{array}{c}\delta_{\theta} \\
{[\%]}\end{array}$ & $\begin{array}{c}\delta_{\text {noise }} \\
{[\%]}\end{array}$ & $\begin{array}{l}\delta_{x_{I P}} \\
{[\%]}\end{array}$ & $\begin{array}{c}\delta_{\beta} \\
{[\%]}\end{array}$ & $\begin{array}{c}\delta_{b g} \\
{[\%]}\end{array}$ & $\begin{array}{c}\delta_{\text {Plug }} \\
{[\%]}\end{array}$ & $\begin{array}{l}\delta_{Q^{2}} \\
{[\%]}\end{array}$ & $\begin{array}{l}\delta_{\text {spa }} \\
{[\%]}\end{array}$ \\
\hline 0.03 & 8.5 & 0.011 & 0.0313 & $\begin{array}{l}10.4 \\
\end{array}$ & 13.8 & 17.3 & 11.2 & -2.1 & 1.2 & -1.5 & -3.0 & 0.8 & -1.3 & -4.1 & 3.0 & 0.2 & $\overline{-1.0}$ \\
\hline 0.03 & 8.5 & 0.017 & 0.0270 & 10.4 & 14.3 & 17.7 & 11.2 & -1.2 & -1.5 & 4.0 & -2.0 & 1.5 & -1.1 & -6.3 & 2.1 & 0.1 & -1.0 \\
\hline 0.03 & 8.5 & 0.027 & 0.0276 & 9.4 & 14.6 & 17.3 & 11.2 & -1.4 & 1.3 & -0.1 & -2.9 & 1.5 & -1.5 & -7.5 & 1.8 & 0.2 & -0.8 \\
\hline 0.03 & 8.5 & 0.043 & 0.0277 & 10.5 & 15.6 & 18.8 & 11.2 & 0.7 & -3.8 & 3.8 & 0.6 & 0.8 & -0.4 & -4.1 & 3.9 & 0.1 & 1.8 \\
\hline 0.03 & 12.0 & 0.0067 & 0.0325 & 17.1 & 16.0 & 23.5 & 11.2 & 2.5 & 1.8 & 0.5 & -5.4 & 0.9 & -1.7 & -5.2 & -0.9 & 0.7 & 0.4 \\
\hline 0.03 & 12.0 & 0.011 & 0.0368 & 12.0 & 12.8 & 17.5 & 11.2 & -0.7 & -1.9 & 2.3 & -2.3 & 0.7 & -1.6 & -3.9 & 1.6 & 0.6 & 1.3 \\
\hline 0.03 & 12.0 & 0.017 & 0.0336 & 11.0 & 14.5 & 18.2 & 11.2 & -2.1 & 0.9 & 2.0 & -3.0 & 1.4 & -1.7 & -6.6 & 3.2 & 0.6 & -0.9 \\
\hline 0.03 & 12.0 & 0.027 & 0.0375 & 11.1 & 14.3 & 18.1 & 11.2 & -1.5 & -3.5 & 2.5 & -1.9 & 1.3 & -1.7 & -6.5 & 2.5 & 0.5 & -0.4 \\
\hline 0.03 & 12.0 & 0.043 & 0.0303 & 11.9 & 14.1 & 18.5 & 11.2 & -1.3 & -0.9 & 1.4 & -2.3 & 1.5 & -0.7 & -5.3 & 2.4 & 0.8 & 1.0 \\
\hline 0.03 & 12.0 & 0.067 & 0.0251 & 12.7 & 13.2 & 18.3 & 11.2 & -1.4 & -1.3 & 1.9 & -2.0 & 1.6 & -0.7 & -4.9 & 1.6 & 0.5 & 0.3 \\
\hline 0.03 & 15.0 & 0.0067 & 0.0462 & 15.6 & 14.6 & 21.4 & 11.2 & -2.1 & 6.5 & 0.9 & -3.6 & 0.6 & -2.3 & -3.6 & 1.3 & -0.2 & -1.7 \\
\hline 0.03 & 15.0 & 0.011 & 0.0354 & 6.6 & 14.7 & 16.1 & 11.2 & -0.9 & -1.7 & -1.3 & -1.7 & 1.4 & -2.0 & -7.5 & 2.1 & 0.4 & -0.8 \\
\hline 0.03 & 15.0 & 0.017 & 0.0360 & 5.4 & 14.1 & 15.1 & 11.2 & 0.4 & 1.2 & 1.5 & -1.6 & 1.4 & -1.9 & -7.2 & 3.0 & 0.3 & 0.6 \\
\hline 0.03 & 15.0 & 0.027 & 0.0317 & 5.4 & 14.2 & 15.2 & 11.2 & -1.2 & -1.4 & 1.5 & -1.2 & 1.2 & -1.6 & -6.7 & 1.8 & 0.3 & 0.3 \\
\hline 0.03 & 15.0 & 0.043 & 0.0271 & 6.7 & 15.1 & 16.5 & 11.2 & -0.7 & 2.3 & 1.2 & -2.2 & 1.5 & -1.6 & -8.7 & 2.6 & 0.1 & 0.5 \\
\hline 0.03 & 15.0 & 0.067 & 0.0244 & 6.4 & 13.9 & 15.3 & 11.2 & -0.7 & 1.1 & 0.4 & -1.2 & 1.6 & -1.2 & -7.2 & 1.6 & 0.4 & -1.0 \\
\hline 0.03 & 15.0 & 0.11 & 0.0239 & 6.1 & 14.9 & 16.1 & 11.2 & -1.1 & -0.7 & 0.7 & -2.8 & 1.6 & -1.3 & -7.8 & 1.8 & 0.2 & -0.2 \\
\hline 0.03 & 20.0 & 0.011 & 0.0307 & 9.7 & 15.1 & 17.9 & 11.2 & -0.3 & -0.6 & 0.9 & -2.0 & 1.3 & -2.4 & -7.7 & 2.7 & 0.6 & 1.1 \\
\hline 0.03 & 20.0 & 0.017 & 0.0391 & 7.3 & 14.4 & 16.1 & 11.2 & 0.4 & -1.1 & -1.2 & -2.5 & 1.1 & -2.0 & -6.7 & 2.6 & 0.6 & 1.3 \\
\hline 0.03 & 20.0 & 0.027 & 0.0321 & 5.9 & 13.4 & 14.6 & 11.2 & -0.6 & -0.8 & 1.3 & -2.3 & 1.1 & -1.6 & -6.2 & 1.0 & 0.6 & 0.7 \\
\hline 0.03 & 20.0 & 0.043 & 0.0292 & 6.2 & 13.5 & 14.9 & 11.2 & 0.7 & -2.2 & 1.3 & -0.1 & 1.1 & -1.3 & -6.4 & 2.1 & 0.6 & -0.8 \\
\hline 0.03 & 20.0 & 0.067 & 0.0285 & 6.0 & 13.3 & 14.6 & 11.2 & -1.1 & -0.8 & 0.6 & -1.9 & 1.1 & -1.2 & -5.8 & 2.5 & 0.6 & -0.7 \\
\hline 0.03 & 20.0 & 0.11 & 0.0235 & 7.0 & 13.7 & 15.4 & 11.2 & -0.5 & -0.4 & -0.8 & -0.9 & 1.6 & -1.1 & -7.0 & 2.1 & 0.6 & -0.5 \\
\hline 0.03 & 25.0 & 0.011 & 0.0348 & 25.7 & 14.1 & 29.3 & 11.2 & -1.3 & 3.1 & 1.5 & -2.9 & 0.3 & -2.1 & -2.9 & 4.8 & 0.2 & 3.3 \\
\hline 0.03 & 25.0 & 0.017 & 0.0385 & 8.3 & 14.3 & 16.5 & 11.2 & 0.6 & 0.8 & 1.3 & -0.8 & 1.4 & -2.0 & -7.6 & 2.0 & 0.6 & 1.2 \\
\hline 0.03 & 25.0 & 0.027 & 0.0384 & 6.4 & 13.5 & 14.9 & 11.2 & -0.1 & -1.5 & 1.7 & -2.4 & 1.3 & -1.5 & -6.1 & 1.8 & 0.5 & 0.1 \\
\hline 0.03 & 25.0 & 0.043 & 0.0305 & 6.5 & 12.5 & 14.1 & 11.2 & -0.6 & -0.6 & 1.1 & -2.2 & 0.9 & -0.8 & -4.0 & 2.4 & 0.3 & 0.6 \\
\hline 0.03 & 25.0 & 0.067 & 0.0274 & 6.7 & 14.8 & 16.2 & 11.2 & -1.0 & -1.1 & 0.9 & -1.7 & 1.4 & -1.5 & -7.9 & 2.4 & 0.7 & -1.1 \\
\hline 0.03 & 25.0 & 0.11 & 0.0258 & 6.2 & 14.1 & 15.4 & 11.2 & -1.4 & -0.8 & 0.9 & -1.1 & 1.7 & -1.2 & -7.6 & 2.2 & 0.6 & -0.5 \\
\hline 0.03 & 25.0 & 0.17 & 0.0265 & 6.4 & 14.6 & 16.0 & 11.2 & 0.5 & 1.0 & 1.0 & -2.2 & 1.7 & -1.4 & -8.2 & 1.6 & 0.5 & -1.1 \\
\hline 0.03 & 35.0 & 0.017 & 0.0533 & 14.7 & 13.7 & 20.1 & 11.2 & -2.7 & 3.8 & 1.2 & -2.9 & 0.4 & -1.7 & -3.2 & -2.7 & 0.7 & 1.9 \\
\hline 0.03 & 35.0 & 0.027 & 0.0415 & 7.7 & 13.0 & 15.1 & 11.2 & 1.3 & 1.6 & 0.5 & -0.7 & 0.8 & -1.3 & -4.7 & 2.9 & 0.8 & 0.4 \\
\hline 0.03 & 35.0 & 0.043 & 0.0411 & 6.9 & 13.1 & 14.8 & 11.2 & -1.1 & -1.7 & 0.3 & -1.5 & 0.7 & -1.0 & -3.8 & 2.0 & 0.6 & -0.6 \\
\hline 0.03 & 35.0 & 0.067 & 0.0312 & 7.1 & 13.9 & 15.6 & 11.2 & 0.9 & -1.2 & 1.1 & -1.2 & 1.3 & -1.3 & -6.7 & 2.9 & 0.9 & 1.2 \\
\hline 0.03 & 35.0 & 0.11 & 0.0311 & 6.7 & 14.3 & 15.8 & 11.2 & -0.9 & -0.6 & 0.6 & -2.2 & 1.6 & -1.2 & -7.8 & 1.7 & 1.0 & -0.9 \\
\hline 0.03 & 35.0 & 0.17 & 0.0258 & 7.0 & 14.3 & 15.9 & 11.2 & -0.6 & 0.9 & 1.4 & -1.3 & 1.8 & -1.0 & -7.3 & 2.6 & 0.9 & -0.3 \\
\hline
\end{tabular}




\begin{tabular}{|c|c|c|c|c|c|c|c|c|c|c|c|c|c|c|c|c|c|c|}
\hline & $x_{\mathbb{P}}$ & $\begin{array}{c}Q^{2} \\
{\left[\mathrm{GeV}^{2}\right]}\end{array}$ & $\beta$ & $x_{\mathbb{P}} \sigma_{r}^{D(3)}$ & $\begin{array}{c}\delta_{\text {stat }} \\
{[\%]}\end{array}$ & $\begin{array}{l}\delta_{\text {sys }} \\
{[\%]}\end{array}$ & $\begin{array}{l}\delta_{t o t} \\
{[\%]}\end{array}$ & $\begin{array}{c}\delta_{\text {unc }} \\
{[\%]}\end{array}$ & $\begin{array}{l}\delta_{l a r} \\
{[\%]}\end{array}$ & $\begin{array}{l}\delta_{\text {ele }} \\
{[\%]}\end{array}$ & $\begin{array}{c}\delta_{\theta} \\
{[\%]}\end{array}$ & $\begin{array}{c}\delta_{\text {noise }} \\
{[\%]}\end{array}$ & $\begin{array}{l}\delta_{x_{I P}} \\
{[\%]}\end{array}$ & $\begin{array}{c}\delta_{\beta} \\
{[\%]} \\
\end{array}$ & $\begin{array}{c}\delta_{b g} \\
{[\%]}\end{array}$ & $\begin{array}{c}\delta_{\text {Plug }} \\
{[\%]}\end{array}$ & $\begin{array}{l}\delta_{Q^{2}} \\
{[\%]}\end{array}$ & $\begin{array}{l}\delta_{s p a} \\
{[\%]}\end{array}$ \\
\hline & 0.03 & 35.0 & 0.27 & 0.0289 & 7.3 & 14.6 & 16.4 & 11.2 & 0.8 & -1.8 & 0.8 & 0.2 & 1.9 & -1.1 & -8.4 & 2.6 & 0.9 & 0.3 \\
\hline & 0.03 & 45.0 & 0.027 & 0.0504 & 11.7 & 13.3 & 17.7 & 11.2 & -1.0 & -1.8 & 1.1 & -2.4 & 0.6 & -1.3 & -3.6 & 2.0 & 0.7 & 0.9 \\
\hline & 0.03 & 45.0 & 0.043 & 0.0402 & 8.6 & 12.6 & 15.3 & 11.2 & 1.4 & -1.1 & 0.2 & -1.6 & 0.8 & -1.1 & -4.2 & 1.3 & 0.6 & -0.8 \\
\hline & 0.03 & 45.0 & 0.067 & 0.0390 & 10.1 & 13.7 & 17.0 & 11.2 & -1.7 & -0.3 & -1.5 & -0.7 & 1.4 & -1.2 & -6.6 & 2.4 & 0.7 & -1.0 \\
\hline & 0.03 & 45.0 & 0.11 & 0.0250 & 8.7 & 14.5 & 16.9 & 11.2 & -0.9 & -1.1 & 1.2 & -1.1 & 1.6 & -1.2 & -8.2 & 2.0 & 0.9 & -0.6 \\
\hline & 0.03 & 45.0 & 0.17 & 0.0260 & 8.2 & 15.3 & 17.4 & 11.2 & 2.1 & 0.5 & 1.4 & -2.3 & 1.9 & -1.3 & -9.2 & 1.5 & 1.0 & -0.1 \\
\hline & 0.03 & 45.0 & 0.27 & 0.0215 & 8.2 & 14.7 & 16.8 & 11.2 & 0.7 & -2.3 & 0.8 & -1.3 & 1.7 & -1.4 & -7.8 & 2.3 & 1.0 & 1.0 \\
\hline & 0.03 & 60.0 & 0.043 & 0.0382 & 13.7 & 13.6 & 19.3 & 11.2 & 1.4 & 1.8 & 2.2 & 1.1 & 1.1 & -1.5 & -5.7 & 2.6 & 1.0 & 2.3 \\
\hline & 0.03 & 60.0 & 0.067 & 0.0387 & 9.4 & 13.4 & 16.4 & 11.2 & 0.8 & -3.1 & 1.1 & 1.3 & 1.2 & -1.2 & -5.6 & 1.7 & 0.7 & 0.5 \\
\hline & 0.03 & 60.0 & 0.11 & 0.0265 & 9.2 & 13.5 & 16.3 & 11.2 & 0.8 & -0.3 & 0.9 & -1.0 & 1.4 & -1.0 & -6.8 & 1.9 & 0.7 & -0.8 \\
\hline & 0.03 & 60.0 & 0.17 & 0.0264 & 8.5 & 16.2 & 18.3 & 11.2 & 1.2 & -1.2 & 1.7 & -1.8 & 2.0 & -1.4 & -10.5 & 3.2 & 1.2 & 0.7 \\
\hline & 0.03 & 60.0 & 0.27 & 0.0222 & 13.6 & 17.0 & 21.8 & 11.2 & -0.5 & -1.0 & 1.0 & 1.8 & 2.3 & -1.3 & -11.6 & 2.3 & 1.3 & 0.3 \\
\hline & 0.03 & 60.0 & 0.43 & 0.0277 & 8.9 & 17.2 & 19.4 & 11.2 & 1.3 & -1.6 & -1.2 & 1.3 & 2.8 & -0.9 & -11.7 & 3.0 & 1.4 & 1.2 \\
\hline & 0.03 & 90.0 & 0.067 & 0.0341 & 33.7 & 19.9 & 39.1 & 11.2 & -0.5 & -4.3 & 1.2 & -5.7 & 1.3 & -1.7 & -5.6 & -3.1 & 1.5 & -0.8 \\
\hline & 0.03 & 90.0 & 0.11 & 0.0337 & 14.4 & 12.7 & 19.2 & 11.2 & 1.6 & -1.3 & -1.1 & -0.5 & 1.2 & -0.7 & -3.9 & 1.8 & 1.0 & 0.3 \\
\hline & 0.03 & 90.0 & 0.17 & 0.0368 & 10.4 & 14.1 & 17.5 & 11.2 & 2.0 & -2.3 & 1.5 & -1.9 & 1.4 & -1.1 & -6.7 & 2.1 & 1.2 & 0.7 \\
\hline & 0.03 & 90.0 & 0.27 & 0.0269 & 10.3 & 13.5 & 17.0 & 11.2 & -0.5 & -1.3 & 1.6 & 0.7 & 1.3 & -1.1 & -6.5 & 1.5 & 1.2 & -0.5 \\
\hline & 0.03 & 90.0 & 0.43 & 0.0328 & 10.6 & 14.0 & 17.5 & 11.2 & -0.3 & -1.6 & -1.5 & -1.3 & 1.4 & -0.6 & -6.2 & 2.2 & 1.1 & -1.3 \\
\hline & 0.03 & 90.0 & 0.67 & 0.0289 & 20.9 & 16.8 & 26.8 & 11.2 & 2.1 & 1.7 & 1.4 & 2.3 & 3.3 & 0.8 & -9.3 & 3.5 & 1.8 & 3.0 \\
\hline
\end{tabular}




\begin{tabular}{|c|c|c|c|c|c|c|c|c|c|c|c|c|c|c|c|c|c|}
\hline$x_{\mathbb{P}}$ & $\begin{array}{c}Q^{2} \\
{\left[\mathrm{GeV}^{2}\right]}\end{array}$ & $\beta$ & $x_{\mathbb{P}} \sigma_{r}^{D(3)}$ & $\begin{array}{c}\delta_{\text {stat }} \\
{[\%]}\end{array}$ & $\begin{array}{l}\delta_{\text {sys }} \\
{[\%]}\end{array}$ & $\begin{array}{l}\delta_{t o t} \\
{[\%]}\end{array}$ & $\begin{array}{c}\delta_{u n c} \\
{[\%]}\end{array}$ & $\begin{array}{l}\delta_{l a r} \\
{[\%]}\end{array}$ & $\begin{array}{l}\delta_{\text {ele }} \\
{[\%]}\end{array}$ & $\begin{array}{c}\delta_{\theta} \\
{[\%]}\end{array}$ & $\begin{array}{c}\delta_{\text {noise }} \\
{[\%]}\end{array}$ & $\begin{array}{l}\delta_{x_{I P}} \\
{[\%]}\end{array}$ & $\begin{array}{c}\delta_{\beta} \\
{[\%]}\end{array}$ & $\begin{array}{c}\delta_{b g} \\
{[\%]}\end{array}$ & $\begin{array}{c}\delta_{P l u g} \\
{[\%]}\end{array}$ & $\begin{array}{c}\delta_{\beta(2)} \\
{[\%]}\end{array}$ & $\begin{array}{l}\delta_{t r a} \\
{[\%]}\end{array}$ \\
\hline 0.01 & 200.0 & 0.32 & 0.0321 & 5.8 & 6.6 & 8.8 & 3.6 & -0.9 & -0.1 & -0.7 & -0.5 & $\overline{1.0}$ & -0.9 & 5.1 & -0.7 & 1.0 & -0.4 \\
\hline 0.01 & 200.0 & 0.5 & 0.0315 & 5.3 & 7.0 & 8.8 & 3.6 & -1.3 & 0.9 & -0.8 & -1.6 & 0.9 & -0.8 & 5.1 & -0.6 & 0.6 & -1.2 \\
\hline 0.01 & 200.0 & 0.8 & 0.0211 & 7.9 & 5.9 & 9.9 & 3.6 & -1.6 & 1.8 & -0.3 & -3.0 & 1.0 & -1.0 & 1.0 & -1.0 & 0.4 & -1.2 \\
\hline 0.01 & 400.0 & 0.8 & 0.0196 & 13.3 & 7.9 & 15.4 & 3.6 & -1.5 & 0.6 & -0.7 & -3.0 & 0.4 & -1.1 & 5.5 & -1.0 & 0.3 & -0.8 \\
\hline 0.03 & 200.0 & 0.11 & 0.0361 & 5.7 & 13.3 & 14.5 & 11.1 & 0.3 & 0.8 & -0.8 & 0.9 & 1.8 & -0.4 & 6.0 & -2.4 & 1.9 & 0.4 \\
\hline 0.03 & 200.0 & 0.17 & 0.0331 & 4.8 & 13.1 & 14.0 & 11.1 & 0.2 & 0.4 & -0.7 & 0.9 & 1.8 & -0.6 & 5.8 & -2.3 & 1.7 & 0.6 \\
\hline 0.03 & 200.0 & 0.27 & 0.0283 & 5.3 & 12.3 & 13.4 & 11.1 & 0.1 & 0.5 & -0.8 & 0.8 & 1.7 & -0.9 & 3.8 & -2.1 & 1.4 & 0.2 \\
\hline 0.03 & 200.0 & 0.43 & 0.0309 & 5.5 & 12.2 & 13.4 & 11.1 & -0.5 & 1.2 & -0.7 & 0.1 & 1.5 & -1.0 & 3.7 & -2.1 & 0.9 & -0.1 \\
\hline 0.03 & 200.0 & 0.67 & 0.0297 & 7.2 & 13.4 & 15.2 & 11.1 & -1.0 & 2.8 & -0.9 & -1.2 & 0.8 & -0.6 & 5.8 & -2.6 & 0.2 & 0.5 \\
\hline 0.03 & 400.0 & 0.27 & 0.0322 & 7.6 & 13.4 & 15.4 & 11.1 & -0.4 & 0.6 & -0.2 & 0.1 & 1.2 & -0.8 & 6.9 & -1.8 & 1.3 & -0.3 \\
\hline 0.03 & 400.0 & 0.43 & 0.0293 & 6.9 & 12.2 & 14.0 & 11.1 & -0.7 & -0.4 & -0.1 & -0.3 & 0.9 & -0.8 & 4.1 & -2.2 & 0.7 & -0.2 \\
\hline 0.03 & 400.0 & 0.67 & 0.0289 & 8.0 & 13.5 & 15.6 & 11.1 & -0.9 & 1.2 & -0.7 & -0.9 & 1.1 & -0.9 & 6.6 & -2.1 & 0.5 & -0.4 \\
\hline 0.03 & 800.0 & 0.43 & 0.0391 & 13.1 & 13.7 & 19.0 & 11.1 & -0.9 & 2.4 & -1.2 & -0.3 & 0.1 & -0.5 & 7.1 & -2.1 & 0.2 & -0.4 \\
\hline 0.03 & 800.0 & 0.67 & 0.0228 & 14.6 & 14.6 & 20.6 & 11.1 & -0.1 & -0.9 & 0.3 & -0.5 & 0.2 & -0.5 & 9.1 & -2.1 & 0.1 & -0.2 \\
\hline 0.03 & 1600.0 & 0.67 & 0.0214 & 27.9 & 15.5 & 31.9 & 11.1 & -1.6 & -5.7 & -3.2 & -1.7 & -0.4 & -0.1 & 7.5 & -2.6 & -0.2 & -0.3 \\
\hline
\end{tabular}

Table 5: Results for $x_{\mathbb{P}} \sigma_{r}^{D}$ at fixed $Q^{2}, \beta$ and $x_{\mathbb{P}}$ (columns 1-4) using data with LAr electrons and $E_{p}=920 \mathrm{GeV}$. Columns 5-7 contain the percentage statistical, systematic and total uncertainties. The remaining columns contain the contributions to the systematic uncertainty from sources which are uncorrelated between data points $\left(\delta_{u n c}\right)$ and the 10 correlated sources leading to the largest uncertainties. These are the LAr hadronic energy scale $\left(\delta_{l a r}\right)$, the LAr electromagnetic energy scale $\left(\delta_{\text {ele }}\right)$, the scattered electron angle measurement $\left(\delta_{\theta}\right)$, the calorimeter noise treatment $\left(\delta_{\text {noise }}\right)$, reweighting the simulation in $x_{\mathbb{P}}\left(\delta_{x_{\mathbb{P}}}\right)$ and $\beta\left(\delta_{\beta}\right)$, the background subtraction using the non-diffractive RAPGAP simulation $\left(\delta_{b g}\right)$, the plug energy scale $\left(\delta_{P l u g}\right)$, the $1-\beta$ reweighting of the simulation $\left(\delta_{\beta(2)}\right)$ and the contribution to the hadronic energy from charged particle tracks $\left(\delta_{t r a}\right)$. Minus signs appear for these systematics if the shift in a variable is anti-correlated rather than correlated with the shift in the cross section. 


\begin{tabular}{|c|c|c|c|c|c|}
\hline$x_{\mathbb{P}}$ range & $\left\langle x_{\mathbb{P}}\right\rangle$ & $\begin{array}{c}\mathrm{d} \sigma / \mathrm{d} x_{\mathbb{P}}[\mathrm{pb}] \\
Q^{2}>200 \mathrm{GeV}^{2}, y<0.9, x_{\mathbb{P}}<0.05\end{array}$ & $\begin{array}{c}\delta_{\text {stat }} \\
(\%)\end{array}$ & $\begin{array}{c}\delta_{\text {tot }} \\
(\%)\end{array}$ & $(\%)$ \\
\hline \hline $0.005-0.016$ & 0.01 & 15 & 59 & 25 & 64 \\
$0.016-0.05$ & 0.03 & 7.7 & 37 & 18 & 41 \\
\hline
\end{tabular}

\begin{tabular}{|c|c|c|c|c|c|}
\hline $\begin{array}{c}Q^{2} \text { range } \\
{\left[\mathrm{GeV}^{2}\right]}\end{array}$ & $\begin{array}{c}\left\langle Q^{2}\right\rangle \\
{\left[\mathrm{GeV}^{2}\right]}\end{array}$ & $\begin{array}{c}\mathrm{d} \sigma / \mathrm{d}^{2}\left[\mathrm{pb} / \mathrm{GeV}^{2}\right] \\
Q^{2}>200 \mathrm{GeV}^{2}, y<0.9, x_{\mathbb{P}}<0.05\end{array}$ & $\begin{array}{c}\delta_{\text {stat }} \\
(\%)\end{array}$ & $\begin{array}{c}\delta_{\text {sys }} \\
(\%)\end{array}$ & $\begin{array}{c}\delta_{\text {tot }} \\
(\%)\end{array}$ \\
\hline \hline $200-560$ & 350 & $6.0 \cdot 10^{-4}$ & 50 & 21 & 54 \\
$560-2240$ & 1150 & $1.0 \cdot 10^{-4}$ & 40 & 18 & 44 \\
\hline
\end{tabular}

\begin{tabular}{|c|c|c|c|c|c|}
\hline$\beta$ range & $\langle\beta\rangle$ & $\begin{array}{c}\mathrm{d} \sigma / \mathrm{d} \beta[\mathrm{pb}] \\
Q_{\text {stat }}\end{array}$ & $\begin{array}{c}\delta_{\text {sys }} \\
(\%)\end{array}$ & $\begin{array}{c}\delta_{\text {tot }} \\
(\%)\end{array}$ \\
\hline \hline $0.3-0.5$ & 0.40 & 0.58 & 62 & 27 & 68 \\
$0.5-0.8$ & 0.65 & 0.44 & 44 & 21 & 48 \\
$0.8-1.0$ & 0.90 & 0.17 & 79 & 17 & 81 \\
\hline
\end{tabular}

Table 6: Measurements of the cross section for the diffractive charged current process $e^{+} p \rightarrow$ $\bar{\nu}_{e} X Y$ for $Q^{2}>200 \mathrm{GeV}^{2}, y<0.9$ and $x_{\mathbb{P}}<0.05$ with $E_{p}=920 \mathrm{GeV}$, differential in $x_{\mathbb{P}}, Q^{2}$ and $\beta$. The differential cross sections correspond to average values over the ranges shown. The percentage statistical, systematic and total uncertainties are also given. 